University of Massachusetts Amherst

ScholarWorks@UMass Amherst

Astronomy Department Faculty Publication Series

Astronomy

1996

\title{
The global rate and efficiency of star formation in spiral galaxies as a function of morphology and environment
}

Judith S. Young

Lori Allen

Jeffrey D.P. Kenney

Amy Lesser

Brooks Rownd

Follow this and additional works at: https://scholarworks.umass.edu/astro_faculty_pubs

Part of the Astrophysics and Astronomy Commons

\section{Recommended Citation}

Young, Judith S.; Allen, Lori; Kenney, Jeffrey D.P.; Lesser, Amy; and Rownd, Brooks, "The global rate and efficiency of star formation in spiral galaxies as a function of morphology and environment" (1996). Astronomical Journal. 859.

Retrieved from https://scholarworks.umass.edu/astro_faculty_pubs/859 


\title{
THE GLOBAL RATE AND EFFICIENCY OF STAR FORMATION IN SPIRAL GALAXIES AS A FUNCTION OF MORPHOLOGY AND ENVIRONMENT
}

\author{
JUdith S. YOUNG, ${ }^{1}$ LORI ALLEN, JeFFREY D. P. KeNNEY, ${ }^{2}$ AMY LESSER, ${ }^{1}$ AND BROOKS ROWND \\ Department of Physics and Astronomy and Five College Radio Astronomy Observatory, University of Massachusetts, Amherst, \\ Massachusetts 01003 \\ Electronic mail: young@phast.umass.edu \\ Received 1996 January 24; revised 1996 June 24
}

\begin{abstract}
CCD images of $\mathrm{H} \alpha$ and $R$-band emission in 120 spiral galaxies were obtained using the now-retired No. 1-0.9 $\mathrm{m}$ telescope of Kitt Peak National Observatory. These images were used to derive the distribution and total flux of continuum-subtracted $\mathrm{H} \alpha$ line emission, and therefore the $\mathrm{H} \alpha$ surface brightnesses and high mass star formation rates in these galaxies. We find a small but significant variation in the mean $\mathrm{H} \alpha$ surface brightness for spiral galaxies along the Hubble sequence; the Sd-Ir galaxies exhibit a mean $\mathrm{H} \alpha$ surface brightness 1.4 times higher than the Sbc-Scd galaxies, and 2-3 times higher than the $\mathrm{Sa}-\mathrm{Sb}$ galaxies. Estimates for the total formation rate for high mass stars have been compared with global molecular gas masses to determine the global efficiency of high mass star formation $\left(=L_{*} / \mathscr{O}_{\mathrm{gas}}\right)$ as a function of morphological type and environment. We find that the mean efficiency of high mass star formation in this sample of spiral galaxies shows little dependence on morphological type for galaxies of type Sa through Scd, although there is a wide range in star formation efficiencies within each type. Galaxies in disturbed environments (i.e., strongly interacting systems) are found to have a mean star formation efficiency $\sim 4$ times higher than in isolated spiral galaxies, uncorrected for extinction. This confirms previous findings (Young et al. 1986a,b; Sanders et al. 1986; Solomon \& Sage 1988; Tinney et al. 1990), based on the far-infrared luminosity rather than the $\mathrm{H} \alpha$ luminosity to trace the rate of high mass star formation, that the mean star formation efficiency among isolated galaxies is significantly lower than that among interacting systems. This result provides further confirmation that the rate of high mass star formation is reasonably well traced by both the $\mathrm{H} \alpha$ and the IR luminosity in spiral galaxies. (c) 1996 American Astronomical Society.
\end{abstract}

\section{INTRODUCTION}

If star formation in external galaxies takes place in molecular clouds, as is observed in the Milky Way, then the evolution of those galaxies is largely driven by the rate at which molecular gas is converted into stars. Among spiral galaxies, it is now well known that molecular gas is found in all spiral types (cf. Young \& Knezek 1989), and it is of great interest to determine whether the disks of galaxies of different Hubble types evolve at different rates. To this end, it is important to determine the mean rate of high mass star formation per unit mass of molecular gas, which we also call the star formation efficiency ( $=L_{*} / \mathscr{M}_{\text {gas }}$ ), for disk galaxies along the Hubble sequence. This approach to the study of spiral galaxies is independent of bulge luminosity-which changes as a function of morphological type (Boroson 1981; Kent 1985)-since the molecular clouds and young stars are both located in the disk.

Previous studies of galaxies have addressed both the morphological type dependence and the environmental dependence of the high mass star formation efficiency through a

${ }^{1}$ Visiting JCMT Fellow, Institue for Astronomy, University of Hawaii, Honolulu, HI 96822.

${ }^{2}$ Department of Astronomy, Yale University, New Haven, CT 06511. comparison of the far-infrared luminosity [L(IR)] with the molecular gas mass $\left[\mathscr{M}\left(\mathrm{H}_{2}\right)\right]$. For early- and late-type spirals, a number of investigators have found no global variations in the mean $L(\mathrm{IR}) / \mathscr{C}\left(\mathrm{H}_{2}\right)$ with type (Rengarajan \& Verma 1986; Young et al. 1989; Thronson et al. 1989; Wiklind \& Henkel 1989; Devereux \& Young 1991). Differences are found, however, in the mean $L(\mathrm{IR}) / \mathscr{M}\left(\mathrm{H}_{2}\right)$ for interacting galaxies relative to isolated spirals (Young et al. 1986a, 1986b; Sanders et al. 1986; Solomon \& Sage 1988; Tinney et al. 1990), indicating that the yield of high mass stars per unit mass of molecular gas in interacting galaxies is $\sim 5$ times higher than in isolated galaxies.

Using the luminosity in $\mathrm{H} \alpha$ as opposed to that in the far-infrared, we have undertaken a program to determine the present rate of high mass star formation in disk galaxies, as well as the star formation rate per unit mass of molecular gas. The primary incentive for this program was originally to determine the distribution of high mass star formation within galaxies, since the IRAS resolution is insufficient to resolve most of the galaxies studied here. Because the flux in $\mathrm{H} \alpha$ is proportional to the number of ionizing photons from $\mathrm{OB}$ stars, in the absence of absorption by dust, it directly traces the presence of recently formed high mass stars. Thus, the ratio of the $\mathrm{H} \alpha$ luminosity to the mass of molecular gas is an 
independent indicator of the yield of high mass stars per unit mass of molecular gas, or the star formation efficiency.

In this paper, we present initial results from our CCD imaging program on the global high mass star formation rate and efficiency in a sample of 120 spiral galaxies. We include a comparison of interacting and isolated galaxies, as well as an examination of the high mass star formation efficiency in spiral galaxies of different morphological types. In a later paper, we will examine the radial variations in the rate of star formation and the yield of high mass stars per unit mass of molecular gas within these galaxies.

\section{THE H $\alpha$ DATA \\ 2.1 Galaxy Sample}

We have obtained $\mathrm{H} \alpha$ and red continuum CCD images for 120 of the 300 galaxies observed as part of the Five College Radio Astronomy Observatory (FCRAO) Extragalactic CO Survey (Young et al. 1995). The galaxies included in the Extragalactic $C O$ Survey cover a wide range in properties, including lenticulars, spirals, and irregulars; galaxies with optical diameters between 4 and $100 \mathrm{kpc}$; environments from pair to cluster, and from isolated to merging systems. The larger sample of galaxies is not complete in a flux-limited sense, nor it is volume limited, but rather the galaxies observed were selected to span a wide range of parameter space. The majority of the galaxies observed as part of the FCRAO Extragalactic CO Survey were selected from the RC2 (de Vaucouleurs et al. 1976) or the IRAS database based on their optical or infrared properties, such as the total blue magnitude, $B_{T}^{0}$, the global $60 \mu \mathrm{m}$ flux density, $S_{60}$, or the global $100 \mu \mathrm{m}$ flux density, $S_{100}$. Most of the galaxies in the $C O$ Survey are spiral or irregular galaxies which have declinations north of $-25^{\circ}$ and satisfy at least one of the following three criteria: (1) $B_{T}^{0}<13.0$, (2) $S_{60}>5 \mathrm{Jy}$, or (3) $S_{100}>10$ Jy (from coadded IRAS data). The galaxies which are included in this CCD imaging program are listed in Table 1 , along with their coordinates, morphological types, blue magnitudes, heliocentric velocities, distances, and optical diameters.

The 120 galaxies which were imaged in $\mathrm{H} \alpha$ include 25 galaxies of type S0-Sab, 44 galaxies of type Sb-Sbc, 20 galaxies of type Sc, 18 galaxies of type Scd-Sdm, 8 Irregulars, and 2 Peculiars. The morphological type distribution of these galaxies is shown in Fig. 1. The galaxies included in this study are found in a wide range of environments. Among the spirals there are six close pairs in which the two galaxies are of roughly equal size, and nine systems which we refer to as merger remnants based on the presence of tidal tails or other morphological peculiarities which can result from galaxy collisions (Schweizer et al. 1983; Joseph \& Wright 1985; Sanders et al. 1986). At the other extreme of environment, 10 galaxies are so isolated that there are no massive companions within $1 \mathrm{Mpc}$ on the sky, 4 are in loose groups, and 43 are members of the Virgo Cluster. Figure 1 also illustrates the morphological type distribution for the Virgo Cluster members included in this imaging study.

\subsection{Imaging Observations}

Measurements of the $\mathrm{H} \alpha+[\mathrm{N} \mathrm{II}]$ emission were made for 120 galaxies using the now-retired No. 1-0.9 m telescope of KPNO ( $f 7.5)$ with either the T12, T13, RCA3, or TEK1 CCD direct imaging system on photometric nights between 1986 October and 1989 April. The CCDs were operated to produce a scale of either $0.77^{\prime \prime}$ or $0.86^{\prime \prime}$ per pixel, depending on the particular CCD. The area imaged was either $6^{\prime}$ by $6^{\prime}$ or $7^{\prime}$ by $4^{\prime}$, also depending on the particular CCD. A summary of the observations is given in Table 2, including the CCD used to image each galaxy, and the characteristics of each CCD.

The $\mathrm{H} \alpha+[\mathrm{N} \mathrm{II}]$ emission line fluxes were obtained by observing each galaxy through two filters: a narrow $(\Delta \lambda$ =76-86 A) interference filter centered on $\mathrm{H} \alpha$ at the velocity of the target galaxy, and either a broad $R$-band filter $(\lambda$ $=6470 \mathrm{~A}, \Delta \lambda=1110 \mathrm{~A})$ or a narrow $R$ filter $(\lambda=7024 \mathrm{~A}$, $\Delta \lambda=380 \mathrm{~A})$ to determine the nearby continuum level. Integration times were typically 5-10 $\mathrm{min}$ in the $R$ band and $1 \mathrm{~h}$ in $\mathrm{H} \alpha$. Airmass corrections were made using the standard extinction curve for Kitt Peak given in the KPNO IRS Standard Star Manual (Barnes \& Hayes 1984). The airmass of the observations, the specific $\mathrm{H} \alpha$ interference filters used, the characteristics of these filters, and the $\mathrm{H} \alpha$ and $R$-band integration times are given in Table 2.

Because of the range in redshifts for the galaxies in our sample, six different $\mathrm{H} \alpha$ interference filters were needed for the imaging observations. For each filter, the transmission characteristics as a function of wavelength were made available to us by the staff at KPNO. Most of the $\mathrm{H} \alpha$ interference filters were characterized by transmission which decreased by less than $5 \%$ for wavelengths within \pm 20 A of the peak, so that no transmission corrections were needed for most of the galaxies. In a few cases, the velocity of a galaxy was such that the redshifted $\mathrm{H} \alpha$ line was inauspiciously located midway between the available filters where the transmission was reduced by $\sim 5 \%-10 \%$, and in these cases a transmission correction was applied to the image.

The images were bias subtracted and flat fielded as described by Schoening (1987) in the "CCD Direct Imaging Camera for the \#1-0.9 m Telescope." After flat fielding, the next step in obtaining continuum-free $\mathrm{H} \alpha+[\mathrm{N} \mathrm{II}]$ images was to subtract the sky emission from each image. Then, the continuum images were registered and scaled relative to the interference filter images using $\sim 5$ unsaturated foreground stars, and the scaled continuum images were subtracted from the interference filter images to yield continuum-free images of the $\mathrm{H} \alpha+[\mathrm{N} \mathrm{II}]$ emission. Figure 2 (Plates 85-90) illustrates the $R$-band and continuum-free $\mathrm{H} \alpha+[\mathrm{N} \mathrm{II}]$ emission in a subset of the galaxies in our sample covering a range in morphological type.

The continuum-subtracted $\mathrm{H} \alpha+[\mathrm{N}$ II] images were flux calibrated using observations from the same night of one or more of the CCD photometric standards G191B2B, Feige $110, \mathrm{HZ} 44$, and $\mathrm{BD}+8^{\circ} 2015$. Table 2 lists the calibration standard used for each galaxy; fluxes for these sources were determined from the KPNO IRS Standard Star Manual (Barnes \& Hayes 1984). The final step in the data reduction procedure consisted of removing incompletely subtracted 
TABLE 1. Galaxy properties.

\begin{tabular}{|c|c|c|c|c|c|c|c|c|c|}
\hline $\begin{array}{c}\text { Galaxy } \\
\text { (1) }\end{array}$ & $\begin{array}{c}\alpha(1950) \\
\text { h m s } \\
\text { (2) }\end{array}$ & $\begin{array}{c}\delta(1950) \\
0, n \\
(3)\end{array}$ & $\begin{array}{l}\text { Type } \\
\text { (4) }\end{array}$ & $\begin{array}{l}\mathrm{B}_{\mathrm{T}}{ }^{\circ} \\
(5)\end{array}$ & $\begin{array}{c}V_{\text {sun }} \\
(\mathrm{km} / \mathrm{sec}) \\
(6)\end{array}$ & $\begin{array}{l}\text { Dist. } \\
(\mathrm{Mpc}) \\
(7)\end{array}$ & $\begin{array}{c}\mathrm{D}_{25} \\
(?) \\
(8)\end{array}$ & $\begin{array}{c}D_{25} \\
(k p c) \\
(9)\end{array}$ & $\begin{array}{l}\text { Alt. } \\
\text { Ref.' } \\
\text { (10) }\end{array}$ \\
\hline $\begin{array}{l}\text { NGC } 157 \\
\text { NGC } 253 \\
\text { NGC } 278 \\
\text { NGC } 488 \\
\text { NGC } 520 \\
\text { NGC } 628 \\
\text { NGC } 660 \\
\text { NGC } 891 \\
\text { NGC } 925 \\
\text { NGC } 972\end{array}$ & $\begin{array}{lll}00 & 32 & 14.4 \\
00 & 45 & 07.8 \\
00 & 49 & 14.8 \\
01 & 19 & 11.2 \\
01 & 21 & 59.4 \\
01 & 34 & 00.7 \\
01 & 40 & 20.7 \\
02 & 19 & 25.2 \\
02 & 24 & 16.6 \\
02 & 31 & 16.6\end{array}$ & $\begin{array}{l}-084018 \\
-253342 \\
+471643 \\
+045936 \\
+033213 \\
+153155 \\
+132332 \\
+420718 \\
+332120 \\
+290535\end{array}$ & $\begin{array}{l}\text { SAB(rs)bc } \\
\text { SAB(s)c } \\
\text { SAB(rs)b } \\
\text { SA(r)b } \\
\text { Pec } \\
\text { SA(s)c } \\
\text { SB(s)a pec } \\
\text { SA(s)b sp } \\
\text { SAB(s)d } \\
\text { I0 }\end{array}$ & $\begin{array}{r}10.67 \\
7.40 \\
10.96 \\
10.83 \\
11.55 \\
9.48 \\
12.28 \\
9.83 \\
10.03 \\
11.53\end{array}$ & $\begin{array}{r}1657 \\
249 \\
642 \\
2180 \\
2168 \\
655 \\
856 \\
524 \\
560 \\
1532\end{array}$ & $\begin{array}{r}35.0 \\
3.3 \\
17.7 \\
45.8 \\
45.4 \\
15.9 \\
19.6 \\
14.1 \\
14.3 \\
33.4\end{array}$ & $\begin{array}{r}4.27 \\
25.12 \\
2.19 \\
5.25 \\
4.79 \\
10.23 \\
9.12 \\
13.49 \\
9.77 \\
3.63\end{array}$ & $\begin{array}{l}43.4 \\
24.1 \\
11.3 \\
70.0 \\
63.3 \\
47.2 \\
52.0 \\
55.4 \\
40.7 \\
35.3\end{array}$ & $\begin{array}{l}\mathbf{a} \\
\mathbf{a}, \mathrm{e}\end{array}$ \\
\hline $\begin{array}{l}\text { NGC } 1055 \\
\text { NGC } 1068 \\
\text { NGC } 1084 \\
\text { NGC } 1087 \\
\text { NGC } 1569 \\
\text { NGC } 1614 \\
\text { NGC } 1961 \\
\text { NGC } 2146 \\
\text { NGC } 2276 \\
\text { NGC } 2336\end{array}$ & $\begin{array}{lll}02 & 39 & 10.7 \\
02 & 40 & 06.5 \\
02 & 43 & 31.8 \\
02 & 43 & 51.6 \\
04 & 26 & 05.8 \\
04 & 31 & 36.0 \\
05 & 36 & 33.9 \\
06 & 10 & 40.1 \\
07 & 10 & 22.0 \\
07 & 18 & 28.0\end{array}$ & $\begin{array}{l}+001345 \\
-001332 \\
-074706 \\
-004219 \\
+644418 \\
-084054 \\
+692116 \\
+782223 \\
+855058 \\
+801635\end{array}$ & $\begin{array}{l}\text { SBb sp } \\
(R) S A(r s) b \\
\text { SA(s)c } \\
\text { SAB(rs)c } \\
\text { IBm } \\
\text { SB(s)c pec } \\
\text { SAB(rs)c } \\
\text { SB(s)ab pec } \\
\text { SAB(rs)c } \\
\text { SAB(r)bc }\end{array}$ & $\begin{array}{r}10.79 \\
9.17 \\
10.73 \\
11.11 \\
10.58 \\
13.15 \\
11.15 \\
10.52 \\
11.44 \\
10.49\end{array}$ & $\begin{array}{r}1050 \\
1109 \\
1413 \\
1824 \\
-87 \\
4745 \\
3890 \\
838 \\
2369 \\
2199\end{array}$ & $\begin{array}{r}21.5 \\
22.7 \\
28.1 \\
36.9 \\
4.7 \\
92.9 \\
81.1 \\
20.6 \\
51.6 \\
47.8\end{array}$ & $\begin{array}{l}7.59 \\
6.92 \\
2.88 \\
3.47 \\
2.88 \\
1.32 \\
4.27 \\
6.03 \\
2.63 \\
6.92\end{array}$ & \begin{tabular}{r|}
47.6 \\
45.7 \\
23.6 \\
37.2 \\
3.9 \\
35.7 \\
100.8 \\
36.1 \\
39.5 \\
96.2
\end{tabular} & $\begin{array}{l}\text { a } \\
\text { e } \\
\text { a }\end{array}$ \\
\hline $\begin{array}{l}\text { NGC } 2339 \\
\text { NGC } 2683 \\
\text { NGC } 2775 \\
\text { NGC } 2798 \\
\text { NGC } 2841 \\
\text { NGC } 2903 \\
\text { NGC } 2976 \\
\text { NGC } 3034 \\
\text { NGC } 3077 \\
\text { NGC } 3079\end{array}$ & $\begin{array}{lll}07 & 05 & 25.1 \\
08 & 49 & 34.8 \\
09 & 07 & 41.0 \\
09 & 14 & 09.5 \\
09 & 18 & 34.9 \\
09 & 29 & 19.9 \\
09 & 43 & 10.0 \\
09 & 51 & 45.3 \\
09 & 59 & 21.9 \\
09 & 58 & 35.4\end{array}$ & $\begin{array}{l}+185142 \\
+333623 \\
+071435 \\
+421237 \\
+511119 \\
+214319 \\
+680843 \\
+695511 \\
+685833 \\
+555511\end{array}$ & $\begin{array}{l}\text { SAB(rs)bc } \\
\text { SA(rs)b } \\
\text { SA(r)ab } \\
\text { SB(s)a pec } \\
\text { SA(r)b } \\
\text { SAB(rs)bc } \\
\text { SAc pec } \\
\text { I0 sp } \\
\text { I0 pec } \\
\text { SB(s)c sp }\end{array}$ & $\begin{array}{r}11.54 \\
9.84 \\
10.85 \\
12.43 \\
9.58 \\
9.05 \\
10.32 \\
8.72 \\
10.28 \\
10.43\end{array}$ & $\begin{array}{r}2423 \\
284 \\
1135 \\
1708 \\
652 \\
569 \\
42 \\
246 \\
10 \\
1137\end{array}$ & $\begin{array}{r}46.7 \\
4.8 \\
19.3 \\
34.2 \\
14.0 \\
9.3 \\
3.5 \\
3.3 \\
3.3 \\
24.2\end{array}$ & $\begin{array}{r}2.75 \\
9.33 \\
4.47 \\
2.75 \\
8.13 \\
12.59 \\
4.90 \\
11.22 \\
4.57 \\
7.59\end{array}$ & \begin{tabular}{|r|r|}
37.3 \\
13.1 \\
25.1 \\
27.3 \\
33.1 \\
34.2 \\
5.0 \\
10.8 \\
4.4 \\
53.5
\end{tabular} & $\begin{array}{l}\text { e } \\
\text { e } \\
\text { e }\end{array}$ \\
\hline $\begin{array}{l}\text { NGC } 3147 \\
\text { NGC } 3184 \\
\text { NGC } 3310 \\
\text { NGC } 3351 \\
\text { NGC } 3504 \\
\text { NGC } 3521 \\
\text { NGC } 3556 \\
\text { NGC } 3593 \\
\text { NGC } 3623 \\
\text { NGC } 3627\end{array}$ & $\begin{array}{llll}10 & 12 & 39.3 \\
10 & 15 & 17.7 \\
10 & 35 & 40.3 \\
10 & 41 & 19.6 \\
11 & 00 & 28.1 \\
11 & 03 & 15.1 \\
11 & 08 & 36.8 \\
11 & 11 & 59.2 \\
11 & 16 & 18.6 \\
11 & 17 & 37.9\end{array}$ & $\begin{array}{l}+733902 \\
+414028 \\
+534545 \\
+115800 \\
+281435 \\
+001358 \\
+555633 \\
+130528 \\
+132200 \\
+131608\end{array}$ & $\begin{array}{l}\text { SA(rs)bc } \\
\text { SAB(rs)cd } \\
\text { SAB(r)bc pec } \\
\text { SB(r)b } \\
\text { (R)SAB(s)ab } \\
\text { SAB(rs)bc } \\
\text { SB(s)cd sp } \\
\text { SA(s)0/a } \\
\text { SAB(rs)a } \\
\text { SAB(s)b }\end{array}$ & $\begin{array}{r}11.07 \\
10.18 \\
10.90 \\
10.18 \\
11.52 \\
9.26 \\
10.00 \\
11.24 \\
9.59 \\
9.26\end{array}$ & $\begin{array}{r}2721 \\
589 \\
994 \\
807 \\
1529 \\
815 \\
685 \\
660 \\
780 \\
697\end{array}$ & \begin{tabular}{r|}
57.6 \\
11.9 \\
21.3 \\
13.5 \\
29.6 \\
12.8 \\
15.4 \\
10.9 \\
6.7 \\
6.7
\end{tabular} & $\begin{array}{r}3.98 \\
6.92 \\
3.63 \\
7.41 \\
2.69 \\
9.55 \\
8.32 \\
5.75 \\
10.00 \\
8.71\end{array}$ & $\begin{array}{l}66.7 \\
23.9 \\
22.4 \\
29.0 \\
23.1 \\
35.6 \\
37.4 \\
18.2 \\
19.5 \\
17.0\end{array}$ & $\begin{array}{l}\text { e } \\
\text { e }\end{array}$ \\
\hline $\begin{array}{l}\text { NGC } 3631 \\
\text { NGC } 3675 \\
\text { NGC } 3690 \\
\text { IC } 694 \\
\text { NGC } 3718 \\
\text { NGC } 3810 \\
\text { NGC } 4038 \\
\text { NGC } 4041 \\
\text { NGC } 4064 \\
\text { NGC } 4088\end{array}$ & $\begin{array}{lll}11 & 18 & 13.2 \\
11 & 23 & 24.2 \\
11 & 25 & 44.2 \\
11 & 25 & 41.8 \\
11 & 29 & 50.7 \\
11 & 38 & 23.5 \\
11 & 59 & 19.2 \\
11 & 59 & 38.7 \\
12 & 01 & 37.3 \\
12 & 03 & 03.1\end{array}$ & $\begin{array}{l}+532643 \\
+435136 \\
+585023 \\
+585000 \\
+532033 \\
+114455 \\
-183506 \\
+622503 \\
+184316 \\
+504913\end{array}$ & $\begin{array}{l}\text { SA(s)c } \\
\text { SA(s)b } \\
\text { IBm pec } \\
\text { SBm pec } \\
\text { SB(s)a pec } \\
\text { SA(rs)c } \\
\text { SB(s)m pec } \\
\text { SA(rs)bc } \\
\text { SB(s)a pec } \\
\text { SAB(rs)bc }\end{array}$ & \begin{tabular}{c|}
10.76 \\
10.49 \\
11.90 \\
$\ldots .$. \\
10.80 \\
10.94 \\
10.88 \\
11.43 \\
11.71 \\
10.60
\end{tabular} & $\begin{array}{r}1167 \\
701 \\
2999 \\
3115 \\
1014 \\
973 \\
1658 \\
1191 \\
1026 \\
742\end{array}$ & $\begin{array}{l}24.9 \\
14.7 \\
62.1 \\
62.1 \\
21.9 \\
17.2 \\
28.9 \\
26.4 \\
20.0 \\
16.4\end{array}$ & $\begin{array}{l}4.57 \\
5.89 \\
2.40 \\
1.17 \\
8.71 \\
4.27 \\
2.58 \\
2.82 \\
4.47 \\
5.75\end{array}$ & $\begin{array}{l}33.1 \\
25.2 \\
43.3 \\
21.1 \\
55.5 \\
21.4 \\
21.7 \\
21.7 \\
26.0 \\
27.5\end{array}$ & a \\
\hline $\begin{array}{l}\text { NGC } 4102 \\
\text { NGC } 4157 \\
\text { NGC } 4178 \\
\text { NGC } 4189 \\
\text { NGC } 4192 \\
\text { NGC } 4194 \\
\text { NGC } 4206 \\
\text { NGC } 4212 \\
\text { NGC } 4216 \\
\text { NGC } 4237\end{array}$ & $\begin{array}{llll}12 & 03 & 51.6 \\
12 & 08 & 34.6 \\
12 & 10 & 13.1 \\
12 & 11 & 13.9 \\
12 & 11 & 15.4 \\
12 & 11 & 41.7 \\
12 & 12 & 43.7 \\
12 & 13 & 06.4 \\
12 & 13 & 20.3 \\
12 & 14 & 38.2\end{array}$ & $\begin{array}{l}+525923 \\
+504551 \\
+110830 \\
+134217 \\
+151023 \\
+544821 \\
+131810 \\
+141045 \\
+132538 \\
+153608\end{array}$ & $\begin{array}{l}\text { SAB(s)b } \\
\text { SAB(s)b sp } \\
\text { SB(rs)dm } \\
\text { SAB(rs)cd } \\
\text { SAB(s)ab } \\
\text { IBm pec } \\
\text { SA(s)bc } \\
\text { SAbc } \\
\text { SAB(s)b } \\
\text { SAB(rs)bc }\end{array}$ & $\begin{array}{l}11.91 \\
11.18 \\
11.38 \\
12.26 \\
10.29 \\
12.55 \\
13.10 \\
11.54 \\
10.26 \\
11.98\end{array}$ & $\begin{array}{r}896 \\
825 \\
297 \\
2097 \\
-129 \\
2528 \\
701 \\
2027 \\
15 \\
945\end{array}$ & $\begin{array}{l}19.7 \\
18.1 \\
20.0 \\
20.0 \\
20.0 \\
52.6 \\
20.0 \\
20.0 \\
20.0 \\
20.0\end{array}$ & $\begin{array}{l}3.16 \\
6.92 \\
5.01 \\
2.45 \\
9.55 \\
2.45 \\
5.25 \\
3.02 \\
8.32 \\
2.29\end{array}$ & $\begin{array}{l}18.1 \\
36.5 \\
29.1 \\
14.3 \\
55.6 \\
37.5 \\
30.5 \\
17.6 \\
48.4 \\
13.3\end{array}$ & $\begin{array}{l}c, d, f \\
f \\
f \\
c, d, f\end{array}$ \\
\hline
\end{tabular}


TABLE 1. (continued)

\begin{tabular}{|c|c|c|c|c|c|c|c|c|c|}
\hline $\begin{array}{c}\text { Galaxy } \\
\text { (1) }\end{array}$ & $\begin{array}{c}\alpha(1950) \\
\text { h m s } \\
(2)\end{array}$ & $\begin{array}{c}\delta(1950) \\
0, " \\
(3)\end{array}$ & $\begin{array}{l}\text { Type } \\
\text { (4) }\end{array}$ & $\begin{array}{l}{ }^{\mathrm{B}_{\mathrm{T}}}{ }^{\circ} \\
(5)\end{array}$ & $\begin{array}{c}V_{\text {sun }} \\
(\mathrm{km} / \mathrm{sec}) \\
(6)\end{array}$ & $\begin{array}{l}\text { Dist. } \\
(\mathrm{Mpc}) \\
(7)\end{array}$ & $\begin{array}{c}\mathrm{D}_{25} \\
(() \\
(8)\end{array}$ & $\begin{array}{c}\mathrm{D}_{25} \\
(\mathbf{k p c}) \\
(9)\end{array}$ & $\begin{array}{l}\text { Alt. } \\
\text { Ref. * } \\
(10)\end{array}$ \\
\hline $\begin{array}{l}\text { NGC } 4254 \\
\text { NGC } 4293 \\
\text { NGC } 4294 \\
\text { NGC } 4298 \\
\text { NGC } 4302 \\
\text { NGC } 4299 \\
\text { NGC } 4303 \\
\text { NGC } 4321 \\
\text { NGC } 4351 \\
\text { NGC } 4380\end{array}$ & $\begin{array}{lll}12 & 16 & 16.9 \\
12 & 18 & 41.1 \\
12 & 18 & 44.8 \\
12 & 19 & 00.4 \\
12 & 19 & 10.2 \\
12 & 18 & 08.0 \\
12 & 19 & 21.4 \\
12 & 20 & 23.2 \\
12 & 21 & 29.5 \\
12 & 22 & 49.6\end{array}$ & $\begin{array}{l}+144146 \\
+183936 \\
+114718 \\
+145303 \\
+145243 \\
+114653 \\
+044458 \\
+160600 \\
+122901 \\
+101733\end{array}$ & $\begin{array}{l}\text { SA(s)c } \\
(R) S B(s) 0 / a \\
\text { SB(s)cd } \\
\text { SA(rs)c } \\
\text { Sc sp } \\
\text { SAB(s)dm } \\
\text { SAB(rs)bc } \\
\text { SAB(s)bc } \\
\text { SB(rs)ab pec } \\
\text { SA(rs)b }\end{array}$ & $\begin{array}{r}10.18 \\
10.77 \\
12.10 \\
11.70 \\
12.67 \\
12.66 \\
9.97 \\
9.86 \\
12.47 \\
13.02\end{array}$ & $\begin{array}{r}2400 \\
882 \\
415 \\
1116 \\
1118 \\
212 \\
1599 \\
1610 \\
2388 \\
964\end{array}$ & $\begin{array}{l}20.0 \\
20.0 \\
20.0 \\
20.0 \\
20.0 \\
20.0 \\
20.0 \\
20.0 \\
20.0 \\
20.0\end{array}$ & $\begin{array}{l}5.37 \\
6.03 \\
3.09 \\
3.16 \\
5.25 \\
1.74 \\
6.03 \\
6.92 \\
2.04 \\
3.72\end{array}$ & $\begin{array}{l}31.2 \\
35.1 \\
18.0 \\
18.4 \\
30.5 \\
10.1 \\
35.1 \\
40.3 \\
11.9 \\
21.6\end{array}$ & $\begin{array}{l}f \\
f \\
f \\
f \\
c, d, f \\
f \\
f \\
f \\
f \\
c, d, f\end{array}$ \\
\hline $\begin{array}{l}\text { NGC } 4394 \\
\text { NGC } 4413 \\
\text { NGC } 4419 \\
\text { NGC } 4424 \\
\text { NGC } 4438 \\
\text { NGC } 4450 \\
\text { NGC } 4459 \\
\text { NGC } 4498 \\
\text { NGC } 4501 \\
\text { NGC } 4519\end{array}$ & $\begin{array}{lll}12 & 23 & 24.7 \\
12 & 23 & 59.7 \\
12 & 24 & 25.1 \\
12 & 24 & 39.0 \\
12 & 25 & 13.5 \\
12 & 25 & 58.0 \\
12 & 26 & 28.3 \\
12 & 29 & 08.8 \\
12 & 29 & 28.1 \\
12 & 30 & 58.1\end{array}$ & $\begin{array}{l}+182930 \\
+125311 \\
+151928 \\
+094151 \\
+131711 \\
+172140 \\
+141520 \\
+170746 \\
+144150 \\
+085548\end{array}$ & $\begin{array}{l}\text { (R)SB(r)b } \\
\text { (R)SB(rs)b } \\
\text { SB(s)a sp } \\
\text { SB(s)a } \\
\text { SA(s)0/a pec } \\
\text { SA(s)ab } \\
\text { SA(s)0+ } \\
\text { SAB(s)cd } \\
\text { SA(rs)b } \\
\text { SB(rs)d }\end{array}$ & \begin{tabular}{r|}
11.51 \\
13.27 \\
11.07 \\
11.84 \\
10.39 \\
10.62 \\
11.07 \\
12.42 \\
9.86 \\
12.30
\end{tabular} & $\begin{array}{r}772 \\
94 \\
-273 \\
450 \\
259 \\
2048 \\
1111 \\
1506 \\
2057 \\
1170\end{array}$ & $\begin{array}{l}20.0 \\
20.0 \\
20.0 \\
20.0 \\
20.0 \\
20.0 \\
20.0 \\
20.0 \\
20.0 \\
20.0\end{array}$ & $\begin{array}{l}3.89 \\
2.45 \\
3.39 \\
3.72 \\
9.33 \\
4.79 \\
3.80 \\
3.24 \\
6.92 \\
3.09\end{array}$ & \begin{tabular}{l|}
22.6 \\
14.3 \\
19.7 \\
21.6 \\
54.3 \\
27.9 \\
22.1 \\
18.8 \\
40.3 \\
18.0
\end{tabular} & $\begin{array}{l}\text { f } \\
\text { c,d,f } \\
c, d, f \\
\text { f } \\
\text { f } \\
\text { f } \\
\text { e } \\
\text { c,d,f } \\
\text { f } \\
\text { f }\end{array}$ \\
\hline $\begin{array}{l}\text { NGC } 4522 \\
\text { NGC } 4526 \\
\text { NGC } 4527 \\
\text { NGC } 4532 \\
\text { NGC } 4535 \\
\text { NGC } 4536 \\
\text { NGC } 4548 \\
\text { NGC } 4561 \\
\text { NGC } 4567 \\
\text { NGC } 4569\end{array}$ & $\begin{array}{lll}12 & 31 & 07.8 \\
12 & 31 & 30.4 \\
12 & 31 & 35.5 \\
12 & 31 & 46.7 \\
12 & 31 & 47.9 \\
12 & 31 & 53.5 \\
12 & 32 & 55.1 \\
12 & 33 & 38.4 \\
12 & 34 & 01.1 \\
12 & 34 & 18.7\end{array}$ & $\begin{array}{l}+092702 \\
+075833 \\
+025545 \\
+064443 \\
+082825 \\
+022750 \\
+144620 \\
+193556 \\
+113201 \\
+132618\end{array}$ & $\begin{array}{l}\text { SB(s)cd sp } \\
\text { SAB(s)0 } \\
\text { SAB(s)bc } \\
\text { IBm } \\
\text { SAB(s)c } \\
\text { SAB(rs)bc } \\
\text { SB(rs)b } \\
\text { SB(rs)dm } \\
\text { SA(rs)bc } \\
\text { SAB(rs)ab }\end{array}$ & $\begin{array}{r}12.98 \\
10.18 \\
10.73 \\
11.76 \\
10.35 \\
10.50 \\
10.71 \\
12.44 \\
11.75 \\
9.80\end{array}$ & $\begin{array}{r}2331 \\
450 \\
1730 \\
2159 \\
1946 \\
1927 \\
468 \\
1474 \\
2199 \\
-312\end{array}$ & $\begin{array}{l}20.0 \\
20.0 \\
20.0 \\
20.0 \\
20.0 \\
20.0 \\
20.0 \\
20.0 \\
20.0 \\
20.0\end{array}$ & $\begin{array}{l}3.72 \\
7.24 \\
6.31 \\
2.88 \\
6.76 \\
7.41 \\
5.37 \\
1.51 \\
2.95 \\
9.55\end{array}$ & \begin{tabular}{|r|}
21.6 \\
42.1 \\
36.7 \\
16.8 \\
39.3 \\
43.1 \\
31.2 \\
8.8 \\
17.2 \\
55.6
\end{tabular} & $\begin{array}{l}c, d, f \\
\text { f } \\
\text { f } \\
\text { f } \\
\text { f } \\
\text { f } \\
\text { f } \\
\text { c,d,f } \\
\text { f } \\
\text { f }\end{array}$ \\
\hline $\begin{array}{l}\text { NGC } 4571 \\
\text { NGC } 4579 \\
\text { NGC } 4639 \\
\text { NGC } 4647 \\
\text { NGC } 4651 \\
\text { NGC } 4654 \\
\text { NGC } 4689 \\
\text { NGC } 4710 \\
\text { NGC } 4713 \\
\text { NGC } 4736\end{array}$ & $\begin{array}{lll}12 & 34 & 25.5 \\
12 & 35 & 12.6 \\
12 & 40 & 21.7 \\
12 & 41 & 01.1 \\
12 & 41 & 12.5 \\
12 & 41 & 25.7 \\
12 & 45 & 15.3 \\
12 & 47 & 09.0 \\
12 & 47 & 25.6 \\
12 & 48 & 32.4\end{array}$ & $\begin{array}{l}+142933 \\
+120540 \\
+133156 \\
+115121 \\
+164005 \\
+132358 \\
+140213 \\
+152615 \\
+053458 \\
+412328\end{array}$ & $\begin{array}{l}\text { SA(r)d } \\
\text { SAB(rs)b } \\
\text { SAB(rs)bc } \\
\text { SAB(rs)c } \\
\text { SA(rs)c } \\
\text { SAB(rs)cd } \\
\text { SA(rs)bc } \\
\text { SA(r)0+ sp } \\
\text { SAB(rs)d } \\
\text { (R)SA(r)ab }\end{array}$ & $\begin{array}{r}13.37 \\
10.33 \\
11.90 \\
11.64 \\
10.99 \\
10.75 \\
12.55 \\
11.25 \\
11.86 \\
8.58\end{array}$ & $\begin{array}{r}348 \\
1805 \\
963 \\
1358 \\
794 \\
1036 \\
1776 \\
1129 \\
664 \\
269\end{array}$ & $\begin{array}{r}20.0 \\
20.0 \\
20.0 \\
20.0 \\
20.0 \\
20.0 \\
20.0 \\
20.0 \\
20.0 \\
6.0\end{array}$ & $\begin{array}{r}3.80 \\
5.37 \\
2.88 \\
3.02 \\
3.80 \\
4.68 \\
3.98 \\
5.13 \\
2.82 \\
10.96\end{array}$ & $\begin{array}{l}22.1 \\
31.2 \\
16.8 \\
17.6 \\
22.1 \\
27.2 \\
23.2 \\
29.8 \\
16.4 \\
21.0\end{array}$ & $\begin{array}{l}\text { c,d,f } \\
\text { f } \\
\text { f } \\
\text { f } \\
\text { f } \\
\text { f } \\
\text { c,d,f } \\
\text { f } \\
\text { f } \\
\text { e }\end{array}$ \\
\hline $\begin{array}{l}\text { NGC } 4808 \\
\text { NGC } 4826 \\
\text { NGC } 5005 \\
\text { NGC } 5194 \\
\text { NGC } 5248 \\
\text { NGC } 5631 \\
\text { NGC } 5907 \\
\text { NGC } 6240 \\
\text { NGC } 6643 \\
\text { NGC } 6946\end{array}$ & $\begin{array}{lll}12 & 53 & 17.0 \\
12 & 54 & 16.9 \\
13 & 08 & 37.6 \\
13 & 27 & 46.9 \\
13 & 35 & 02.4 \\
14 & 25 & 00.0 \\
15 & 14 & 34.8 \\
16 & 50 & 27.6 \\
18 & 21 & 13.3 \\
20 & 33 & 48.8\end{array}$ & $\begin{array}{l}+043428 \\
+215718 \\
+371925 \\
+472716 \\
+090823 \\
+564800 \\
+563033 \\
+022906 \\
+743243 \\
+595850\end{array}$ & $\begin{array}{l}\text { SA(s)cd } \\
\text { (R)SA(rs)ab } \\
\text { SAB(rs)bc } \\
\text { SA(s)bc pec } \\
\text { SAB(rs)bc } \\
\text { SA(s)0 } \\
\text { SA(s)c sp } \\
\text { I0 pec } \\
\text { SA(rs)c } \\
\text { SAB(rs)cd }\end{array}$ & $\begin{array}{r}12.03 \\
8.96 \\
10.19 \\
8.62 \\
10.49 \\
12.22 \\
10.08 \\
13.80 \\
11.07 \\
8.49\end{array}$ & $\begin{array}{r}773 \\
397 \\
1015 \\
460 \\
1146 \\
2144 \\
592 \\
7503 \\
1482 \\
46\end{array}$ & $\begin{array}{r}20.0 \\
7.5 \\
21.4 \\
9.6 \\
22.0 \\
46.2 \\
15.6 \\
151.9 \\
34.7 \\
10.1\end{array}$ & $\begin{array}{r}2.69 \\
9.33 \\
5.37 \\
10.96 \\
6.46 \\
2.19 \\
12.30 \\
2.19 \\
3.89 \\
10.96\end{array}$ & $\begin{array}{l}15.6 \\
20.5 \\
33.4 \\
30.6 \\
41.4 \\
29.4 \\
55.8 \\
96.8 \\
39.3 \\
32.2\end{array}$ & $\begin{array}{l}\text { c,f } \\
\mathrm{e}\end{array}$ \\
\hline $\begin{array}{l}\text { NGC } 6951 \\
\text { NGC } 7217 \\
\text { NGC } 7331 \\
\text { NGC } 7479 \\
\text { NGC } 7541 \\
\text { NGC } 7625 \\
\text { Arp } 220 \\
\text { IC } 342 \\
\text { IC } 356 \\
\text { Mrk } 231\end{array}$ & $\begin{array}{lll}20 & 36 & 37.7 \\
22 & 05 & 37.6 \\
22 & 34 & 47.7 \\
23 & 02 & 26.8 \\
23 & 12 & 10.3 \\
23 & 18 & 00.6 \\
15 & 32 & 47.3 \\
03 & 41 & 58.6 \\
04 & 02 & 34.5 \\
12 & 54 & 05.0\end{array}$ & $\begin{array}{l}+655548 \\
+310653 \\
+340935 \\
+120306 \\
+041543 \\
+165715 \\
+234006 \\
+675626 \\
+694046 \\
+570841\end{array}$ & $\begin{array}{l}\text { SAB(rs)bc } \\
(R) S A(r) a b \\
\text { SA(s)bc } \\
\text { SB(s)c } \\
\text { SB(rs)bc pec } \\
\text { SA(rs)a pec } \\
\text { Pec } \\
\text { SAB(rs)cd } \\
\text { SA(s)ab pec } \\
\text { SA(rs)c pec }\end{array}$ & $\begin{array}{r}11.25 \\
10.49 \\
9.51 \\
11.33 \\
11.85 \\
12.47 \\
14.02 \\
7.86 \\
10.35 \\
13.86\end{array}$ & $\begin{array}{r}1343 \\
946 \\
826 \\
2385 \\
2672 \\
1637 \\
5400 \\
32 \\
822 \\
12430\end{array}$ & $\begin{array}{r}32.5 \\
24.5 \\
22.1 \\
52.1 \\
57.2 \\
37.3 \\
110.2 \\
4.5 \\
20.3 \\
251.1\end{array}$ & $\begin{array}{r}3.80 \\
3.72 \\
10.72 \\
4.07 \\
3.47 \\
1.78 \\
2.00 \\
17.78 \\
5.25 \\
1.74\end{array}$ & $\begin{array}{r}36.0 \\
26.6 \\
68.9 \\
61.7 \\
57.7 \\
19.3 \\
64.1 \\
23.6 \\
31.0 \\
127.1\end{array}$ & $\begin{array}{l}\text { b,c,d,g } \\
e\end{array}$ \\
\hline
\end{tabular}


*See Young et al. (1995) for alternate references.

Col. (1) Galaxy name-NGC, IC, Mrk, or Arp designation.

Col. (2)-(3) Right Ascension and Declination (1950.0 coordinates) from Dressel and Condon (1976), unless alternate reference ' $a$ ' is noted in Column (10).

Col. (4) Morphological type from RC2, unless alternate reference ' $b$ ' is noted in Column (10).

Col. (5) Total blue magnitude, $\mathrm{B}_{\mathrm{T}}{ }^{\circ}$, corrected for galactic and internal absorption from RC2, unless alternate reference ' $c$ ' is noted in Column (10).

Col. (6) Heliocentric velocity from RC2, unless alternate reference ' $d$ ' is noted in Column (10).

Col. (7) Distance to the galaxy. For most galaxies, the distance was calculated from the Hubble Law after applying the solar motion correction to the velocity (RC2, column 29), and using $\mathrm{H}_{\mathrm{o}}=50 \mathrm{~km} \mathrm{~s}^{-1} \mathrm{Mpc}^{-1}$. The exceptions include a small number of nearby galaxies for which the alternate reference 'e' is noted in Column (10), and Virgo cluster members, assumed to be located at a distance of $20.0 \mathrm{Mpc}$ [see alternate reference ' $\mathrm{f}$ ' in Column (10)].

Col. (8) Optical angular diameter measured in arcminutes out to the $25 \mathrm{mag} \mathrm{arcsec}-2$ isophote from $\mathrm{RC} 2$, unless alternate reference ' $\mathrm{g}$ ' is noted in Column (10).

Col. (9) Linear diameter in kiloparsecs, calculated using Columns (7) and (8).

Col. (10) Alternate References:

$\mathrm{a}=$ Coordinates from RC2, Lonsdale et al. (1985), Sanders et al. (1986), Tacconi and Young (1987), Bothun et al. (1989), Giovanelli and Haynes (1989), or Goldsmith and Young (1989).

$b=$ Morphological type from UGC or Tully (1988).

$\mathrm{c}=\mathrm{B}_{\mathrm{T}}$ from UGC and corrected for galactic and internal absorption as in RC2, or $\mathbf{B}_{\mathrm{T}}{ }^{\circ}$ from de Vaucouleurs, de Vaucouleurs and Buta (1981) or Tacconi and Young (1987).

d =Heliocentric velocity from Huchtmeier et al. (1983), Tully (1988), Thronson et al. (1989), or Bothun et al. (1989).

$\mathrm{e}=$ For nearby galaxies, distances are not derived from the Hubble Law. These distances are from Rogstad and Shostak (1972), Sandage and Tammann

(1975), Bosma et al. (1977), Hunter, Gallagher, and Rautenkranz (1982), Tacconi and Young (1985, 1987), Thronson et al. (1989), or Young et al. (1989). $\mathrm{f}=$ Galaxies in the Virgo cluster are taken to be at a distance of $20.0 \mathrm{Mpc}$, following Kenney and Young (1988).

$\mathrm{g}=\mathrm{D}_{25}$ from UGC, Tully (1988), Tacconi and Young (1987), or Bothun et al. (1989).

foreground stars and cosmic rays from the flux-calibrated images, using IRAF. Calibrated $\mathrm{H} \alpha+[\mathrm{N} \mathrm{II}]$ emission line fluxes were determined over the area in which $\mathrm{H}$ II regions were detected in each image. Table 2 lists the observed $\mathrm{H} \alpha$ $+[\mathrm{N} \mathrm{II}]$ fluxes for the program galaxies.

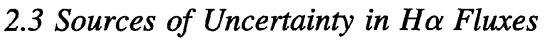

Possible sources of error in the $\mathrm{H} \alpha$ fluxes include (1) contamination by $[\mathrm{N} \mathrm{II}]$ emission, since the $\mathrm{H} \alpha$ interference

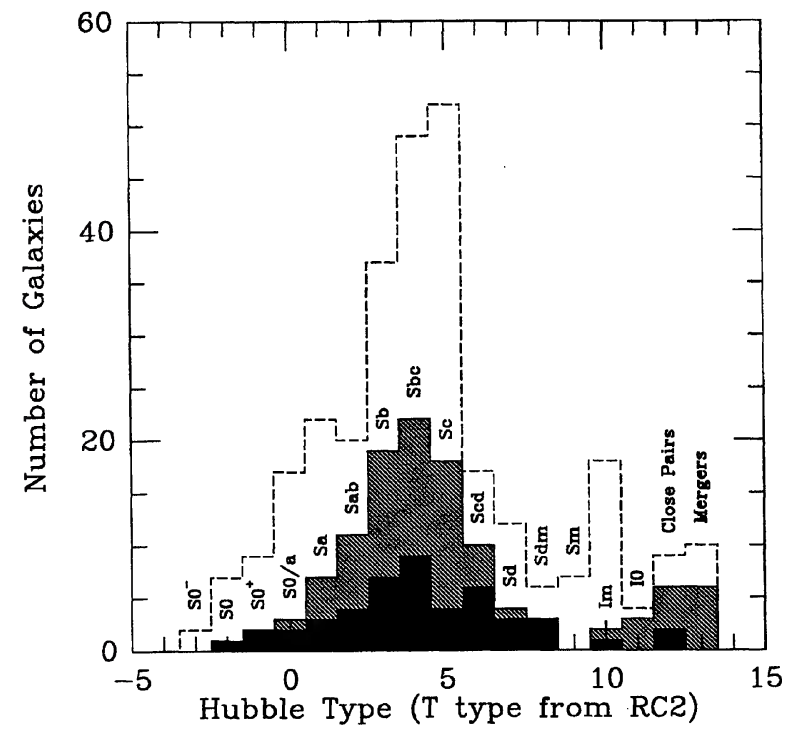

FIG. 1. The distribution of morphological types for the 120 galaxies in the $\mathrm{H} \alpha$ imaging survey (grey histogram), and for the 43 of these galaxies in the Virgo Cluster (black histogram). Morphological types were taken from the RC2 (see Table 1). The dashed histogram indicates the type distribution for the 300 galaxies in the FCRAO Extragalactic CO Survey, from which the galaxies in this imaging study were chosen. filters are $\sim 80 \mathrm{~A}$ wide and therefore include the satellite [N II] lines at $\lambda \lambda 6548,6584,(2)$ determination of the continuum level to subtract from the observed $\mathrm{H} \alpha$ interference filter image, (3) absolute calibration uncertainties, and (4) extinction, both Galactic and internal to the galaxies themselves. Additionally, some $\mathrm{H} \alpha$ emission could arise from an active nucleus, as opposed to on-going star formation. Each of these possibilities will be discussed in turn in this section.

\subsection{1 [N II] emission}

The $[\mathrm{N} \mathrm{I}] / \mathrm{H} \alpha$ ratio is likely to be high in the nuclei of spiral galaxies where $\mathrm{H} \alpha$ absorption is strongest and [N II] is in emission (Rubin \& Ford 1986). However, the galaxies in our sample are not as nearby as M31 and M33, and Rubin \& Ford (1986) show that the nuclear $\mathrm{H} \alpha$ absorption is overshadowed by disk $\mathrm{H} \alpha$ emission in more distant galaxies observed with a fixed aperture. Indeed, Kennicutt \& Kent (1983) find that nuclear [N II] emission rarely dominates the integrated photometry of spiral galaxies. They also find, from a compilation of published $\mathrm{H}$ II region spectrophotometry for 14 spiral and 7 irregular galaxies, that global [N II] $\mathrm{H} \alpha$ variations introduce only a small dispersion in the observed equivalent widths ( $\pm 5 \%$ to $20 \%$ in the extremes). Thus, the $[\mathrm{N} \mathrm{II}]$ lines in these galaxies generally contribute a small fraction of the observed $\mathrm{H} \alpha+[\mathrm{N}$ II] emission line flux. We have made no correction for this emission, and hereafter we will refer to the $\mathrm{H} \alpha+[\mathrm{N} \mathrm{II}]$ measurements as $\mathrm{H} \alpha$ fluxes.

One effect which may tend to compensate for the contribution of the $[\mathrm{N} \mathrm{II}]$ lines to the observed flux is related to the fact that the broad $R$-band filter, which was used for most of the continuum observations, covers a wavelength range which includes the $\mathrm{H} \alpha$ and [N II] lines. This 1110 A wide continuum filter has a bandwidth which is $\sim 14$ times larger than the bandwidth of the $\sim 80$ A wide interference filters, 
TABLE 2. Observational parameters.

\begin{tabular}{|c|c|c|c|c|c|c|c|c|c|}
\hline $\begin{array}{c}\text { Galaxy } \\
\text { (1) }\end{array}$ & $\begin{array}{l}\text { Obs. Date } \\
\text { (m/d/yr) } \\
\text { (2) }\end{array}$ & (3) & $\begin{array}{c}\mathbf{R} \\
\text { Airmass } \\
\text { (4) }\end{array}$ & $\begin{array}{c}\mathbf{R} \\
\text { Image } \\
(\mathrm{sec}) \\
(5)\end{array}$ & $\begin{array}{c}\mathrm{H} \alpha \\
\text { Airmass } \\
\text { (6) }\end{array}$ & $\begin{array}{c}\text { H } \alpha \\
\text { Image } \\
(\mathrm{sec}) \\
(7)\end{array}$ & $\begin{array}{c}\mathrm{H \alpha} \\
\text { Filter } \\
(8)\end{array}$ & Calibrator & $\begin{array}{c}\log F(H \alpha) \\
\left(\mathrm{erg} / \mathrm{cm}^{2} / \mathrm{s}\right) \\
(10)\end{array}$ \\
\hline $\begin{array}{l}\text { NGC } 157 \\
\text { NGC } 253 \\
\text { NGC } 278 \\
\text { NGC } 488 \\
\text { NGC } 520 \\
\text { NGC } 628 \\
\text { NGC } 660 \\
\text { NGC } 891 \\
\text { NGC } 925 \\
\text { NGC } 972\end{array}$ & $\begin{array}{l}12 / 09 / 87 \\
12 / 03 / 88 \\
12 / 11 / 87 \\
12 / 12 / 87 \\
10 / 25 / 86 \\
12 / 03 / 88 \\
10 / 26 / 86 \\
12 / 10 / 87 \\
12 / 01 / 88 \\
02 / 25 / 89\end{array}$ & $\begin{array}{c}\text { T13 } \\
\text { RCA3 } \\
\text { T13 } \\
\text { T13 } \\
\text { T12 } \\
\text { RCA3 } \\
\text { T12 } \\
\text { T13 } \\
\text { RCA3 } \\
\text { TEK1 }\end{array}$ & $\begin{array}{l}1.35 \\
1.86 \\
1.07 \\
1.17 \\
1.14 \\
1.05 \\
1.07 \\
1.05 \\
1.01 \\
1.85\end{array}$ & $\begin{array}{c}500 \\
200 \\
170 \\
200 \\
2000 * \\
450 \\
1100 * \\
600 \\
300 \\
300\end{array}$ & $\begin{array}{l}1.38 \\
1.90 \\
1.07 \\
1.15 \\
1.18 \\
1.08 \\
1.05 \\
1.04 \\
1.04 \\
2.25\end{array}$ & $\begin{array}{l}4000 \\
3600 \\
1600 \\
2000 \\
4000 \\
3000 \\
4500 \\
2500 \\
3000 \\
2700\end{array}$ & $\begin{array}{l}2 \\
1 \\
1 \\
2 \\
2 \\
1 \\
2 \\
1 \\
1 \\
2\end{array}$ & $\begin{array}{l}\text { Feige } 110 \\
\text { Feige } 110 \\
\text { Feige } 110 \\
\text { Feige } 110 \\
\text { G191B2B } \\
\text { Feige } 110 \\
\text { G191B2B } \\
\text { Feige 110 } \\
\text { G191B2B } \\
\text { G191B2B }\end{array}$ & $\begin{array}{l}-11.11 \\
-10.19 \\
-11.17 \\
-12.29 \\
-12.40 \\
-10.91 \\
-11.64 \\
-11.68 \\
-11.10 \\
-11.51\end{array}$ \\
\hline $\begin{array}{l}\text { NGC } 1055 \\
\text { NGC } 1068 \\
\text { NGC } 1084 \\
\text { NGC } 1087 \\
\text { NGC } 1569 \\
\text { NGC } 1614 \\
\text { NGC } 1961 \\
\text { NGC } 2146 \\
\text { NGC } 2276 \\
\text { NGC } 2336\end{array}$ & $\begin{array}{l}12 / 10 / 87 \\
10 / 25 / 86 \\
12 / 01 / 88 \\
12 / 03 / 88 \\
12 / 11 / 87 \\
12 / 09 / 87 \\
02 / 26 / 89 \\
10 / 25 / 86 \\
12 / 01 / 88 \\
12 / 03 / 88\end{array}$ & $\begin{array}{c}\text { TI3 } \\
\text { TI2 } \\
\text { RCA3 } \\
\text { RCA3 } \\
\text { TI3 } \\
\text { TI3 } \\
\text { TEK1 } \\
\text { TI2 } \\
\text { RCA3 } \\
\text { RCA3 }\end{array}$ & $\begin{array}{l}1.27 \\
1.20 \\
1.32 \\
1.20 \\
1.19 \\
1.34 \\
1.36 \\
1.46 \\
1.71 \\
1.58\end{array}$ & $\begin{array}{c}500 \\
40 * \\
300 \\
400 \\
125 \\
100 \\
300 \\
900 \\
300 \\
300\end{array}$ & $\begin{array}{l}1.25 \\
1.20 \\
1.31 \\
1.24 \\
1.21 \\
1.32 \\
1.37 \\
1.45 \\
1.70 \\
1.55\end{array}$ & $\begin{array}{r}5000 \\
80 \\
1800 \\
3600 \\
2500 \\
1000 \\
3000 \\
3600 \\
2700 \\
3000\end{array}$ & $\begin{array}{l}1 \\
2 \\
2 \\
2 \\
1 \\
3 \\
3 \\
1 \\
2 \\
2\end{array}$ & $\begin{array}{l}\text { Feige } 110 \\
\text { G191B2B } \\
\text { G191B2B } \\
\text { Feige } 110 \\
\text { Feige } 110 \\
\text { Feige } 110 \\
\text { G191B2B } \\
\text { G191B2B } \\
\text { G191B2B } \\
\text { Feige } 110\end{array}$ & $\begin{array}{l}-12.03 \\
-10.34 \\
-11.14 \\
-11.36 \\
-10.64 \\
-11.84 \\
-11.52 \\
-11.27 \\
-11.26 \\
-11.02\end{array}$ \\
\hline $\begin{array}{l}\text { NGC } 2339 \\
\text { NGC } 2683 \\
\text { NGC } 2775 \\
\text { NGC } 2798 / 9 \\
\text { NGC } 2841 \\
\text { NGC } 2903 \\
\text { NGC } 2976 \\
\text { NGC } 3034 \\
\text { NGC } 3077 \\
\text { NGC } 3079\end{array}$ & $\begin{array}{l}02 / 27 / 89 \\
12 / 12 / 87 \\
12 / 03 / 88 \\
12 / 03 / 88 \\
12 / 02 / 88 \\
12 / 02 / 88 \\
12 / 01 / 88 \\
12 / 01 / 88 \\
03 / 23 / 87 \\
10 / 26 / 86\end{array}$ & $\begin{array}{c}\text { TEK1 } \\
\text { TI3 } \\
\text { RCA3 } \\
\text { RCA3 } \\
\text { RCA3 } \\
\text { RCA3 } \\
\text { RCA3 } \\
\text { RCA3 } \\
\text { TI2 } \\
\text { T12 }\end{array}$ & $\begin{array}{l}1.08 \\
1.08 \\
1.43 \\
1.06 \\
1.06 \\
1.08 \\
1.24 \\
1.42 \\
1.31 \\
1.58\end{array}$ & $\begin{array}{l}300 \\
300 \\
300 \\
200 \\
120 \\
300 \\
300 \\
100 \\
1300 * \\
1500 *\end{array}$ & $\begin{array}{l}1.05 \\
1.12 \\
1.35 \\
1.04 \\
1.06 \\
1.07 \\
1.24 \\
1.39 \\
1.38 \\
1.46\end{array}$ & $\begin{array}{l}3000 \\
4500 \\
3600 \\
3000 \\
1800 \\
2500 \\
1900 \\
1200 \\
1200 \\
3600\end{array}$ & $\begin{array}{l}2 \\
1 \\
2 \\
2 \\
1 \\
1 \\
1 \\
1 \\
1 \\
2\end{array}$ & $\begin{array}{c}\text { G191B2B } \\
\text { G191B2B } \\
\text { Feige 110 } \\
\text { Feige 110 } \\
\text { G191B2B } \\
\text { G191B2B } \\
\text { G191B2B } \\
\text { G191B2B } \\
\text { BD+82015 } \\
\text { G191B2B }\end{array}$ & $\begin{array}{l}-11.65 \\
-11.23 \\
-11.63 \\
-11.67 \\
-11.42 \\
-10.67 \\
-11.04 \\
-10.04 \\
-11.22 \\
-11.47\end{array}$ \\
\hline $\begin{array}{l}\text { NGC } 3147 \\
\text { NGC } 3184 \\
\text { NGC } 3310 \\
\text { NGC } 3351 \\
\text { NGC } 3504 \\
\text { NGC } 3521 \\
\text { NGC } 3556 \\
\text { NGC } 3593 \\
\text { NGC } 3623 \\
\text { NGC } 3627\end{array}$ & $\begin{array}{l}12 / 08 / 87 \\
12 / 11 / 87 \\
12 / 08 / 87 \\
03 / 26 / 88 \\
12 / 03 / 88 \\
12 / 11 / 87 \\
12 / 09 / 87 \\
02 / 27 / 89 \\
12 / 11 / 87 \\
12 / 10 / 87\end{array}$ & $\begin{array}{c}\text { T13 } \\
\text { T13 } \\
\text { T13 } \\
\text { T12 } \\
\text { RCA3 } \\
\text { TI3 } \\
\text { T13 } \\
\text { TEK1 } \\
\text { TI3 } \\
\text { T13 }\end{array}$ & $\begin{array}{l}1.40 \\
1.44 \\
1.08 \\
1.40 \\
1.12 \\
1.19 \\
1.61 \\
1.35 \\
1.25 \\
1.20\end{array}$ & $\begin{array}{r}300 \\
450 \\
200 \\
200 \\
150 \\
100 \\
750 \\
450 \\
70 \\
180\end{array}$ & $\begin{array}{l}1.35 \\
1.39 \\
1.08 \\
1.34 \\
1.11 \\
1.20 \\
1.41 \\
1.23 \\
1.28 \\
1.12\end{array}$ & $\begin{array}{r}4100 \\
4500 \\
2000 \\
2500 \\
750 \\
300 \\
5000 \\
4000 \\
1400 \\
1800\end{array}$ & $\begin{array}{l}2 \\
1 \\
1 \\
1 \\
2 \\
1 \\
1 \\
1 \\
1 \\
1\end{array}$ & $\begin{array}{l}\text { Feige } 110 \\
\text { Feige } 110 \\
\text { Feige } 110 \\
\text { G191B2B } \\
\text { Feige } 110 \\
\text { Feige } 110 \\
\text { Feige } 110 \\
\text { G191B2B } \\
\text { Feige } 110 \\
\text { Feige } 110\end{array}$ & $\begin{array}{l}-11.81 \\
-11.12 \\
-10.81 \\
-11.09 \\
-11.40 \\
-10.81 \\
-11.21 \\
-11.73 \\
-11.43 \\
-10.75\end{array}$ \\
\hline $\begin{array}{l}\text { NGC } 3631 \\
\text { NGC } 3675 \\
\text { NGC } 3690 / \\
\text { IC } 694 \\
\text { NGC } 3718 \\
\text { NGC } 3810 \\
\text { NGC } 4038 / 9 \\
\text { NGC } 4041 \\
\text { NGC } 4064 \\
\text { NGC } 4088\end{array}$ & $\begin{array}{l}12 / 02 / 88 \\
02 / 27 / 89 \\
12 / 11 / 87 \\
12 / 03 / 88 \\
12 / 09 / 87 \\
12 / 12 / 87 \\
02 / 27 / 89 \\
04 / 01 / 89 \\
12 / 10 / 87\end{array}$ & $\begin{array}{c}\text { RCA3 } \\
\text { TEK1 } \\
\text { T13 } \\
\text { RCA3 } \\
\text { T13 } \\
\text { TI3 } \\
\text { TEK1 } \\
\text { TEK1 } \\
\text { T13 }\end{array}$ & $\begin{array}{l}1.12 \\
1.61 \\
1.38 \\
\\
1.12 \\
1.35 \\
1.68 \\
1.24 \\
1.38 \\
1.14\end{array}$ & $\begin{array}{l}300 \\
200 \\
150 \\
\\
300 \\
450 \\
300 \\
400 \\
420 \\
300\end{array}$ & $\begin{array}{l}1.15 \\
1.35 \\
1.61 \\
1.21 \\
1.27 \\
1.08\end{array}$ & $\begin{array}{r}3000 \\
3000 \\
1500 \\
3000 \\
3600 \\
600 \\
3000 \\
4000 \\
3300\end{array}$ & $\begin{array}{l}2 \\
1 \\
2 \\
2 \\
1 \\
1\end{array}$ & $\begin{array}{l}\text { G191B2B } \\
\text { G191B2B } \\
\text { Feige 110 } \\
\text { Feige 110 } \\
\text { Feige 110 } \\
\text { Feige 110 } \\
\text { G191B2B } \\
\text { G191B2B } \\
\text { Feige 110 }\end{array}$ & $\begin{array}{l}-11.13 \\
-11.21 \\
-11.09 \\
\\
-12.54 \\
-11.15 \\
-10.79 \\
-11.25 \\
-12.11 \\
-11.17\end{array}$ \\
\hline $\begin{array}{l}\text { NGC } 4102 \\
\text { NGC } 4157 \\
\text { NGC } 4178 \\
\text { NGC } 4189 \\
\text { NGC } 4192 \\
\text { NGC } 4194 \\
\text { NGC } 4206 \\
\text { NGC } 4212 \\
\text { NGC } 4216 \\
\text { NGC } 4237\end{array}$ & $\begin{array}{l}12 / 12 / 87 \\
12 / 12 / 87 \\
03 / 23 / 87 \\
03 / 26 / 88 \\
03 / 31 / 89 \\
12 / 11 / 87 \\
04 / 02 / 89 \\
03 / 22 / 87 \\
04 / 01 / 89 \\
03 / 30 / 89\end{array}$ & $\begin{array}{c}\text { TI3 } \\
\text { TI3 } \\
\text { TI2 } \\
\text { TI2 } \\
\text { TEK1 } \\
\text { TI3 } \\
\text { TEK1 } \\
\text { TI2 } \\
\text { TEK1 } \\
\text { TEK1 }\end{array}$ & $\begin{array}{l}1.38 \\
1.25 \\
1.60 \\
1.49 \\
1.64 \\
1.26 \\
1.88 \\
1.05 \\
1.14 \\
1.77\end{array}$ & $\begin{array}{c}80 \\
300 \\
3300 * \\
450 \\
420 \\
125 \\
420 \\
3000 * * \\
250 \\
420\end{array}$ & $\begin{array}{l}1.25 \\
1.22 \\
1.28 \\
1.30 \\
1.44 \\
1.20 \\
1.59 \\
1.09 \\
1.10 \\
1.52\end{array}$ & $\begin{array}{r}600 \\
4500 \\
3900 \\
4500 \\
4000 \\
1250 \\
4000 \\
3000 \\
2500 \\
4000\end{array}$ & $\begin{array}{l}1 \\
1 \\
1 \\
2 \\
1 \\
3 \\
1 \\
1 \\
1 \\
1\end{array}$ & $\begin{array}{l}\text { Feige } 110 \\
\text { Feige } 110 \\
\text { BD+82015 } \\
\text { G191B2B } \\
\text { G191B2B } \\
\text { Feige 110 } \\
\text { G191B2B } \\
\text { BD+82015 } \\
\text { G191B2B } \\
\text { G191B2B }\end{array}$ & $\begin{array}{l}-11.72 \\
-11.59 \\
-11.59 \\
-11.90 \\
-11.43 \\
-11.70 \\
-12.04 \\
-11.73 \\
-11.52 \\
-12.15\end{array}$ \\
\hline
\end{tabular}


TABLE 2. (continued)

\begin{tabular}{|c|c|c|c|c|c|c|c|c|c|}
\hline (1) & $\begin{array}{l}\text { Obs. Date } \\
(\mathrm{m} / \mathrm{d} / \mathrm{yr}) \\
(2)\end{array}$ & (3) & $\begin{array}{c}\text { R } \\
\text { Airmass } \\
\text { (4) }\end{array}$ & $\begin{array}{c}\mathbf{R} \\
\text { Image } \\
\text { (sec) } \\
\text { (5) }\end{array}$ & $\begin{array}{c}\mathrm{H \alpha} \\
\text { Airmass } \\
\text { (6) }\end{array}$ & $\begin{array}{c}\mathrm{H} \alpha \\
\text { Image } \\
(\mathrm{sec}) \\
(7)\end{array}$ & $\begin{array}{c}\mathrm{H} \alpha \\
\text { Filter } \\
\text { (8) }\end{array}$ & (9) & $\begin{array}{c}\log \mathrm{F}(\mathrm{H} \alpha) \\
\left(\mathrm{erg} / \mathrm{cm}^{2} / \mathrm{s}\right) \\
(10)\end{array}$ \\
\hline $\begin{array}{l}\text { NGC } 4254 \\
\text { NGC } 4293 \\
\text { NGC } 4294 \\
\text { NGC } 4298 / \\
\text { NGC } 4302 \\
\text { NGC } 4299 \\
\text { NGC } 4303 \\
\text { NGC } 4321 \\
\text { NGC } 4351 \\
\text { NGC } 4380\end{array}$ & $\begin{array}{l}03 / 24 / 87 \\
03 / 25 / 88 \\
03 / 31 / 89 \\
03 / 31 / 89 \\
03 / 30 / 89 \\
03 / 29 / 88 \\
03 / 24 / 87 \\
03 / 31 / 89 \\
04 / 01 / 89\end{array}$ & $\begin{array}{c}\text { T12 } \\
\text { TI2 } \\
\text { TEK1 } \\
\text { TEK1 } \\
\\
\text { TEK1 } \\
\text { TI2 } \\
\text { TI2 } \\
\text { TEK1 } \\
\text { TEK1 }\end{array}$ & $\begin{array}{l}1.31 \\
1.17 \\
1.26 \\
1.07 \\
\\
1.30 \\
1.25 \\
1.84 \\
1.08 \\
1.10\end{array}$ & $\begin{array}{l}1000 * \\
300 \\
420 \\
420 \\
420 \\
200 \\
900 * \\
420 \\
420\end{array}$ & $\begin{array}{l}1.21 \\
1.32 \\
1.17 \\
1.06 \\
\\
\\
1.17 \\
1.16 \\
1.59 \\
1.13 \\
1.09\end{array}$ & $\begin{array}{l}3000 \\
3600 \\
4000 \\
4000 \\
\\
4000 \\
2000 \\
2000 \\
4000 \\
4000\end{array}$ & $\begin{array}{l}1 \\
2 \\
2 \\
2 \\
1\end{array}$ & $\begin{array}{l}\text { BD+82015 } \\
\text { G191B2B } \\
\text { G191B2B } \\
\text { G191B2B } \\
\\
\text { G191B2B } \\
\text { G191B2B } \\
\text { BD+82015 } \\
\text { G191B2B } \\
\text { G191B2B }\end{array}$ & $\begin{array}{l}-10.89 \\
-11.92 \\
-11.71 \\
-11.74 \\
\\
-11.62 \\
-10.66 \\
-11.09 \\
-12.43 \\
-12.28\end{array}$ \\
\hline $\begin{array}{l}\text { NGC } 4394 \\
\text { NGC } 4413 \\
\text { NGC } 4419 \\
\text { NGC } 4424 \\
\text { NGC } 4438 \\
\text { NGC } 4450 \\
\text { NGC } 4459 \\
\text { NGC } 4498 \\
\text { NGC } 4501 \\
\text { NGC } 4519\end{array}$ & $\begin{array}{l}03 / 30 / 89 \\
03 / 26 / 88 \\
03 / 26 / 87 \\
04 / 02 / 89 \\
04 / 02 / 89 \\
04 / 01 / 89 \\
03 / 30 / 89 \\
04 / 01 / 89 \\
03 / 29 / 88 \\
03 / 26 / 88\end{array}$ & $\begin{array}{l}\text { TEK1 } \\
\text { TI2 } \\
\text { TI2 } \\
\text { TEK1 } \\
\text { TEK1 } \\
\text { TEK1 } \\
\text { TEK1 } \\
\text { TEK1 } \\
\text { TI2 } \\
\text { TI2 }\end{array}$ & $\begin{array}{l}1.05 \\
1.07 \\
1.83 \\
1.40 \\
1.06 \\
1.06 \\
1.06 \\
1.25 \\
1.89 \\
1.13\end{array}$ & $\begin{array}{l}420 \\
900 \\
600 * \\
420 \\
420 \\
300 \\
250 \\
420 \\
250 \\
600\end{array}$ & $\begin{array}{l}1.05 \\
1.08 \\
1.61 \\
1.28 \\
1.50 \\
1.11 \\
1.11 \\
1.43 \\
1.70 \\
1.15\end{array}$ & $\begin{array}{l}4000 \\
4500 \\
1200 \\
4000 \\
3000 \\
3000 \\
3000 \\
4000 \\
2000 \\
3600\end{array}$ & $\begin{array}{l}1 \\
1 \\
1 \\
1 \\
1 \\
2 \\
2 \\
2 \\
2 \\
2\end{array}$ & $\begin{array}{c}\text { G191B2B } \\
\text { G191B2B } \\
\text { HZ44 } \\
\text { G191B2B } \\
\text { G191B2B } \\
\text { G191B2B } \\
\text { G191B2B } \\
\text { G191B2B } \\
\text { G191B2B } \\
\text { G191B2B }\end{array}$ & $\begin{array}{l}-11.97 \\
-12.16 \\
-11.81 \\
-12.10 \\
-11.63 \\
-12.18 \\
-12.39 \\
-12.02 \\
-11.14 \\
-11.74\end{array}$ \\
\hline $\begin{array}{l}\text { NGC } 4522 \\
\text { NGC } 4526 \\
\text { NGC } 4527 \\
\text { NGC } 4532 \\
\text { NGC } 4535 \\
\text { NGC } 4536 \\
\text { NGC } 4548 \\
\text { NGC } 4561 \\
\text { NGC } 4567 / 8 \\
\text { NGC } 4569\end{array}$ & $\begin{array}{l}04 / 02 / 89 \\
03 / 25 / 88 \\
03 / 25 / 87 \\
03 / 26 / 88 \\
03 / 29 / 88 \\
03 / 30 / 89 \\
03 / 29 / 88 \\
03 / 26 / 87 \\
03 / 31 / 89 \\
03 / 25 / 88\end{array}$ & $\begin{array}{l}\text { TEK1 } \\
\text { TI2 } \\
\text { TI2 } \\
\text { TI2 } \\
\text { TI2 } \\
\text { TEK1 } \\
\text { TI2 } \\
\text { TI2 } \\
\text { TEK1 } \\
\text { TI2 }\end{array}$ & $\begin{array}{l}1.16 \\
1.16 \\
1.23 \\
1.23 \\
1.67 \\
1.29 \\
1.05 \\
1.03 \\
1.23 \\
1.15\end{array}$ & $\begin{array}{l}420 \\
120 \\
450 * \\
900 \\
300 \\
400 \\
300 \\
500 \\
420 \\
150\end{array}$ & $\begin{array}{l}1.12 \\
1.17 \\
1.22 \\
1.15 \\
1.46 \\
1.45 \\
1.06 \\
1.31 \\
1.40 \\
1.10\end{array}$ & $\begin{array}{l}4000 \\
2000 \\
2500 \\
3600 \\
2500 \\
4000 \\
2700 \\
6600 \\
4000 \\
3000\end{array}$ & $\begin{array}{l}2 \\
1 \\
2 \\
2 \\
2 \\
2 \\
1 \\
2 \\
2 \\
1\end{array}$ & $\begin{array}{c}\text { G191B2B } \\
\text { G191B2B } \\
\text { BD+82015 } \\
\text { G191B2B } \\
\text { G191B2B } \\
\text { G191B2B } \\
\text { G191B2B } \\
\text { HZA4 } \\
\text { G191B2B } \\
\text { G191B2B }\end{array}$ & $\begin{array}{l}-12.29 \\
-11.23 \\
-11.28 \\
-11.35 \\
-11.26 \\
-11.34 \\
-12.07 \\
-11.98 \\
-11.38 \\
-11.24\end{array}$ \\
\hline $\begin{array}{l}\text { NGC } 4571 \\
\text { NGC } 4579 \\
\text { NGC } 4639 \\
\text { NGC } 4647 \\
\text { NGC } 4651 \\
\text { NGC } 4654 \\
\text { NGC } 4689 \\
\text { NGC } 4710 \\
\text { NGC } 4713 \\
\text { NGC } 4736\end{array}$ & $\begin{array}{l}03 / 23 / 87 \\
03 / 29 / 88 \\
03 / 31 / 89 \\
03 / 30 / 89 \\
03 / 23 / 87 \\
03 / 26 / 87 \\
03 / 25 / 87 \\
03 / 26 / 87 \\
03 / 26 / 87 \\
03 / 30 / 88\end{array}$ & $\begin{array}{c}\text { TI2 } \\
\text { TI2 } \\
\text { TEK1 } \\
\text { TEK1 } \\
\text { TI2 } \\
\text { T12 } \\
\text { TI2 } \\
\text { TI2 } \\
\text { T12 } \\
\text { T12 }\end{array}$ & $\begin{array}{l}1.09 \\
1.07 \\
1.66 \\
1.51 \\
2.01 \\
2.14 \\
1.06 \\
1.36 \\
1.18 \\
1.25\end{array}$ & $\begin{array}{c}2500 * \\
250 \\
420 \\
350 \\
900 * \\
500 \\
2500 * \\
300 \\
800 \\
90\end{array}$ & $\begin{array}{l}1.05 \\
1.06 \\
2.26 \\
1.87 \\
1.68 \\
1.74 \\
1.29 \\
1.11 \\
1.12 \\
1.22\end{array}$ & $\begin{array}{l}4500 \\
1500 \\
4000 \\
4000 \\
2500 \\
4000 \\
4000 \\
1850 \\
3800 \\
1500\end{array}$ & $\begin{array}{l}1 \\
2 \\
1 \\
2 \\
1 \\
2 \\
2 \\
2 \\
1 \\
1\end{array}$ & $\begin{array}{c}\text { BD+82015 } \\
\text { G191B2B } \\
\text { G191B2B } \\
\text { G191B2B } \\
\text { BD+82015 } \\
\text { HZA4 } \\
\text { BD+82015 } \\
\text { HZ44 } \\
\text { HZ44 } \\
\text { G191B2B }\end{array}$ & $\begin{array}{l}-11.91 \\
-11.49 \\
-11.79 \\
-11.67 \\
-11.62 \\
-11.50 \\
-11.84 \\
-11.39 \\
-11.52 \\
-10.27\end{array}$ \\
\hline $\begin{array}{l}\text { NGC } 4808 \\
\text { NGC } 4826 \\
\text { NGC } 5005 \\
\text { NGC } 5194 \\
\text { NGC } 5248 \\
\text { NGC } 5631 \\
\text { NGC } 5907 \\
\text { NGC } 6240 \\
\text { NGC } 6643 \\
\text { NGC } 6946\end{array}$ & $\begin{array}{l}03 / 23 / 87 \\
02 / 27 / 89 \\
02 / 27 / 89 \\
02 / 26 / 89 \\
02 / 27 / 89 \\
04 / 02 / 89 \\
03 / 30 / 88 \\
03 / 29 / 88 \\
03 / 30 / 88 \\
12 / 02 / 88\end{array}$ & $\begin{array}{l}\text { TI2 } \\
\text { TEK1 } \\
\text { TEK1 } \\
\text { TEK1 } \\
\text { TEK1 } \\
\text { TEK1 } \\
\text { T12 } \\
\text { T12 } \\
\text { T12 } \\
\text { RCA3 }\end{array}$ & $\begin{array}{l}1.20 \\
1.10 \\
1.01 \\
1.50 \\
1.17 \\
1.27 \\
1.10 \\
1.44 \\
1.39 \\
1.26\end{array}$ & $\begin{array}{c}1500 * \\
120 \\
120 \\
300 \\
300 \\
300 \\
1000 \\
400 \\
450 \\
300\end{array}$ & $\begin{array}{l}1.27 \\
1.07 \\
1.02 \\
1.38 \\
1.16 \\
1.31 \\
1.11 \\
1.36 \\
1.38 \\
1.31\end{array}$ & $\begin{array}{l}4000 \\
1500 \\
1500 \\
3000 \\
3600 \\
1500 \\
5900 \\
1500 \\
1800 \\
1500\end{array}$ & $\begin{array}{l}1 \\
1 \\
2 \\
1 \\
2 \\
2 \\
1 \\
1 \\
5 \\
2 \\
1\end{array}$ & $\begin{array}{l}\text { BD+82015 } \\
\text { G191B2B } \\
\text { G191B2B } \\
\text { G191B2B } \\
\text { G191B2B } \\
\text { G191B2B } \\
\text { G191B2B } \\
\text { G191B2B } \\
\text { G191B2B } \\
\text { G191B2B }\end{array}$ & $\begin{array}{r}-11.63 \\
-11.08 \\
-11.38 \\
-10.33 \\
-11.15 \\
<-17.00 \\
-11.48 \\
-11.64 \\
-11.41 \\
-10.47\end{array}$ \\
\hline $\begin{array}{l}\text { NGC } 6951 \\
\text { NGC } 7217 \\
\text { NGC } 7331 \\
\text { NGC } 7479 \\
\text { NGC } 7541 \\
\text { NGC } 7625 \\
\text { Arp } 220 \\
\text { IC } 342 \\
\text { IC } 356 \\
\text { Mrk } 231\end{array}$ & $\begin{array}{l}12 / 03 / 88 \\
12 / 01 / 88 \\
12 / 10 / 87 \\
10 / 25 / 86 \\
10 / 26 / 86 \\
12 / 01 / 88 \\
03 / 25 / 88 \\
12 / 02 / 88 \\
12 / 08 / 87 \\
03 / 30 / 88\end{array}$ & $\begin{array}{c}\text { RCA3 } \\
\text { RCA3 } \\
\text { TI3 } \\
\text { TI2 } \\
\text { TI2 } \\
\text { RCA3 } \\
\text { TI2 } \\
\text { RCA3 } \\
\text { TI3 } \\
\text { TI2 }\end{array}$ & $\begin{array}{l}1.33 \\
1.07 \\
1.03 \\
1.08 \\
1.18 \\
1.11 \\
1.05 \\
1.27 \\
1.28 \\
1.20\end{array}$ & $\begin{array}{c}300 \\
150 \\
100 \\
1200 \\
2000 * \\
300 \\
900 \\
100 \\
300 \\
150\end{array}$ & $\begin{array}{l}1.39 \\
1.12 \\
1.06 \\
1.10 \\
1.14 \\
1.17 \\
1.06 \\
1.29 \\
1.29 \\
1.19\end{array}$ & $\begin{array}{r}3000 \\
1800 \\
600 \\
3600 \\
4500 \\
2500 \\
4200 \\
800 \\
5400 \\
300\end{array}$ & $\begin{array}{l}2 \\
2 \\
1 \\
2 \\
2 \\
2 \\
4 \\
1 \\
1 \\
6\end{array}$ & $\begin{array}{l}\text { Feige } 110 \\
\text { Feige } 110 \\
\text { Feige 110 } \\
\text { G191B2B } \\
\text { G191B2B } \\
\text { Feige 110 } \\
\text { G191B2B } \\
\text { G191B2B } \\
\text { Feige 110 } \\
\text { G191B2B }\end{array}$ & $\begin{array}{l}-11.67 \\
-11.66 \\
-11.10 \\
-11.57 \\
-11.60 \\
-11.76 \\
-12.70 \\
-10.93 \\
-11.83 \\
-11.73\end{array}$ \\
\hline
\end{tabular}


Notes to Table 2

Col. (1) Galaxy name-NGC, IC, Mrk, or Arp designation.

Col. (2) Date of the imaging observations.

Col. (3) CCD used for the observations on the now-retired \#1-0.9 $\mathrm{m}$ telescope at KPNO. The TI CCDs were operated in a $2 \times 2$ pixel-summed mode. The CCD characteristics are as follows:

\begin{tabular}{lccc}
\hline \hline CCD & Number of Pixels & Pixel Scale & Field \\
\hline T12 & $396 \times 396$ & $0.86^{\prime \prime} /$ pixel & $5.7^{\prime} \times 2.7^{\prime}$ \\
TI3 & $396 \times 396$ & $0.86^{\prime \prime} /$ pixel & $5.7^{\prime} \times 5.7^{\prime}$ \\
RCA3 & $508 \times 320$ & $0.86^{\prime \prime} /$ pixel & $7.3^{\prime}(\mathrm{E}-\mathrm{W}) \times 4.6^{\prime}(\mathrm{N}-\mathrm{S})$ \\
TEK1 & $512 \times 512$ & $0.77^{\prime \prime} /$ pixel & $6.6^{\prime} \times 6.6^{\prime}$ \\
\hline \hline
\end{tabular}

Col. (4) The airmass of the observations made using the R-band filter. The airmass correction used as part of the flux calibration of the R-band image is given by $10^{0.4 K_{\lambda} \mathrm{x}}$, where $\mathrm{x}$ is the airmass and $\mathrm{K}_{\lambda}$ is the extinction at wavelength $\lambda$ given by the standard extinction curve for Kitt Peak (Barnes and Hayes 1984). For the broad R-band filter used for most of the continuum observations, $K_{\lambda}$ has a value of 0.101 . For galaxies in which a narrow R-band filter was used to image the continuum, $K_{\lambda}$ has a value of 0.073 ; galaxies for which this is the case have longer $R$-band integration times and are indicated with an asterisk in column 5 .

Col. (5) Integration time, in seconds, for the R-band continuum image. The broad R-band filter has a central wavelength of $6470 \mathrm{~A}$ and a width of $1110 \mathrm{~A}$. Longer integrations were made using a narrow R-band filter for some galaxies, indicated by an asterisk following the time. The narrow R-band filter has a central wavelength of 7024 A and a width of 380 A. For NGC 4212, indicated by a double asterisk (**), the continuum was observed through the filter indicated as $\mathrm{H} \alpha-6$ in the note to Column (8).

Col. (6) The airmass of the observations made using the $\mathrm{H} \alpha$ interference filter. The airmass correction used as $\mathrm{p}$ art of the flux calibration of the H $\alpha$ image is given by $10^{0.4 \mathrm{~K}_{\lambda} \mathrm{x}}$, where $\mathrm{x}$ is the airmass and $\mathrm{K}_{\lambda}$ is the extinction at wavelength $\lambda$ given by the standard extinction curve for Kitt Peak (Barnes and Hayes 1984). For the $\mathrm{H} \alpha$ interference filters used for the imaging observations, $K_{\lambda}$ has a value ranging between 0.095 and 0.082 depending on the central wavelength of the filter. Individual values of $K_{\lambda}$ are given for each $H \alpha$ filter in the note to column (8).

Col. (7) Integration time, in seconds, for the $\mathrm{H} \alpha$ interference filter image.

Col. (8) The $\mathrm{H} \alpha$ filters used for the imaging observations depend on the velocity of the galaxy, and include the following:

\begin{tabular}{ccccc}
\hline \hline $\begin{array}{c}\mathrm{H} \alpha \\
\text { filter }\end{array}$ & $\begin{array}{c}\text { Central } \\
\text { Wavelength } \\
\text { (A) }\end{array}$ & $\begin{array}{c}\text { Filter } \\
\text { Width } \\
(\mathrm{A})\end{array}$ & $\begin{array}{c}\text { Velocity Range } \\
(\mathrm{km} / \mathrm{sec})\end{array}$ & $\begin{array}{c}\text { Extinction } \\
\text { Coefficient } \\
\mathrm{K}_{\lambda}\end{array}$ \\
\hline 1 & 6563 & 80 & $0 \pm 1600$ & 0.095 \\
2 & 6608 & 76 & $1965 \pm 1590$ & 0.093 \\
3 & 6649 & 70 & $3880 \pm 1580$ & 0.091 \\
4 & 6693 & 81 & $5942 \pm 1850$ & 0.089 \\
5 & 6744 & 86 & $8274 \pm 1965$ & 0.087 \\
6 & 6826 & 80 & $12,022 \pm 1828$ & 0.082 \\
\hline \hline
\end{tabular}

Col. (9) Standard star used for flux calibration of the $\mathrm{H} \alpha$ and R-band continuum emission. Fluxes were determined from the KPNO IRS Standard Star Manual of Barnes and Hayes (1984).

Col. (10) Logarithm of the observed $\mathrm{H} \alpha+[\mathrm{NII}]$ flux in units of $\mathrm{erg} \mathrm{cm}^{-2} \mathrm{~s}^{-1}$. No corrections for extinction have been made. The uncertainty in $\mathrm{F}(\mathrm{H} \alpha)$ is estimated to be $\sim 20 \%$. For the galaxy pairs-NGC 2798/2799, Arp 299 (NGC 3690/IC 694), NGC 4038/4039, NGC 4298/4302, NGC 4567/4568-the flux listed is that for both galaxies. For the galaxies with the largest angular sizes (i.e., NGC 253 and IC 342), the fluxes listed are for the inner 6' region only.

and a transmissivity at $\mathrm{H} \alpha$ which is similar to that of the interference filters. Thus, the continuum filter detects not only the red continuum, but also $\sim 7 \%$ of the $\mathrm{H} \alpha$ line emission which is detected in the interference filter images. As a result, when the continuum image is subtracted from the interference filter image, there is a slight oversubtraction of the line emission. However, this oversubtraction of the line emission will tend to balance the presence of the [N II] lines in the first place, so no correction for this effect was made. A larger source of uncertainty is the actual determination of the continuum level, which can lead to a $\pm 20 \%$ uncertainty in the global $\mathrm{H} \alpha$ flux, as discussed below.

\subsubsection{Determination of the continuum level}

Determination of the continuum level in the $\mathrm{H} \alpha$ interference filter image can introduce an error in the $\mathrm{H} \alpha$ flux, because a single scale factor is applied to the continuum image.
(While this is not the ideal situation, the only way to improve upon it is to obtain a spectrum at every location and determine the continuum level at every pixel.) In general, we find that there is a $\pm 5 \%$ error in the determination of the continuum level, which can lead to an uncertainty of up to $\pm 20 \%$ in the global $\mathrm{H} \alpha$ flux for low surface brightness extended emission.

\subsubsection{Absolute calibration}

The absolute calibration of the emission line images depends on the repeatability of individual standard star measurements and the airmass correction based on the Kitt Peak standard extinction curve. From observations of the same standard stars at different airmasses and on different nights, we find that the calibration measurements are repeatable with high accuracy, and that the uncertainty in the absolute calibration is $\pm 5 \%$. All of the observations presented here were taken on photometric nights. 


\subsubsection{Extinction}

The $\mathrm{H} \alpha$ fluxes listed in Table 2 have not been corrected for Galactic extinction, but that correction has been applied to the $\mathrm{H} \alpha$ luminosities listed in Table 3 (see Sec. 3.1). Although no correction for extinction within the galaxies has been made for the entire sample, Kennicutt \& Kent (1983) suggest that the extinction within the galaxies in their sample is typically $\sim 1 \mathrm{mag}$. This indicates that the $\mathrm{H} \alpha$ fluxes and luminosities we measure are lower limits to the intrinsic $\mathrm{H} \alpha$ fluxes and luminosities, and therefore lower limits to the derived rates of high mass star formation.

The extinction of the $\mathrm{H} \alpha$ emission in a galaxy will tend to be higher for galaxies which are more highly inclined to the line of sight. In Fig. 3, we show the distribution of galaxy inclinations as a function of morphological type for the galaxies we have imaged in $\mathrm{H} \alpha$. This figure illustrates that most of the galaxies in our study have inclinations less than $70^{\circ}$, although a wide range of inclinations was included.

We have derived internal extinction corrections for five of the most heavily reddened galaxies in the present sample: NGC 520, NGC 660, NGC 1068, NGC 2146, and M82. These corrections are based on near-IR imaging of [S III] $\lambda$ 9532, which suffers less extinction than does the $\mathrm{H} \alpha$ (Waller et al. 1988; Young et al. 1988). The amount of extinction was derived from the $[\mathrm{S} \mathrm{HI}] / \mathrm{H} \alpha$ ratio, conservatively assuming an intrinsic (unreddened) ratio of 0.5; the intrinsic [S III]/H $\alpha$ ratio is 0.4 in Orion (Lester et al. 1979) and 0.33 in a sample of 8 compact H II regions (Dennefeld \& Stasinska 1985). Thus, the adopted value of 0.5 for the unreddened [S III]/H $\alpha$ ratio may result in underestimating the extinction. Extinction maps derived in this way for the heavily obscured central regions of these five galaxies lead to global extinction corrections which range from a factor of 1.7 in NGC 1068 to a factor of 26 in NGC 520, consistent with independent measurements of the extinction in the central regions (Benvenuti et al. 1975).

Calculations by Rubin (1988) indicate that the unreddened $[\mathrm{S} \mathrm{III}] / \mathrm{H} \alpha$ ratio is approximately constant over a range of a factor of $\sim 2$ in temperature and density and $\sim 10$ in metallicity. Waller et al. (1992) find that the unreddened ratio depends on metal abundance (varying by a factor of $\sim 3$ from Galactic to Magellanic metallicity), with a dispersion of only $25 \%$ for a given metal abundance. The results presented here do not include the extinction corrections for NGC 520, $660,1068,2146$, and M82 except where noted.

\subsection{Comparison with $\mathrm{H \alpha}$ Fluxes in the Literature}

There are several published surveys of $\mathrm{H} \alpha$ emission in galaxies (Cohen 1976; Bagnuolo 1976; Huchra 1977; Bushouse 1987; Pogge \& Eskridge 1987), the largest of which are by Kennicutt \& Kent (1983; hereafter KK) and Kennicutt et al. (1987; hereafter K87). Figure 4 shows a comparison of the $\mathrm{H} \alpha$ fluxes measured by us with those of $\mathrm{KK}$ and $\mathrm{K} 87$ for the 45 galaxies in common between these samples. The KK measurements were made using large aperture ( $1^{\prime}$ to $\left.7^{\prime}\right)$ photometry, while the $\mathrm{K} 87$ fluxes were obtained from images taken with either a video camera or a CCD. All of the measurements were made with filters which also include the [N II] lines.

The mean ratio of the flux we measure to the flux in the other studies is $1.2 \pm 0.2$. This indicates that the data sets are consistent at a level which is within the uncertainties in the individual fluxes.

\section{GALAXY LUMINOSITIES AND MASSES}

\subsection{H $\alpha$ Luminosities}

The $\mathrm{H} \alpha$ fluxes derived from the CCD imaging have been used to derive $\mathrm{H} \alpha$ luminosities for the program galaxies. The $\mathrm{H} \alpha$ luminosity is given by

$$
L(\mathrm{H} \alpha)=4 \pi D^{2} f(\mathrm{H} \alpha) C,
$$

where $D$ is the distance to the galaxy, $f(\mathrm{H} \alpha)$ is the observed $\mathrm{H} \alpha$ flux listed in Table 2, and $C$ is the correction for extinction in our Galaxy. This extinction, in magnitudes, is assumed to be of the form $A(\mathrm{H} \alpha)=0.08[\csc |b|-1]$, where $b$ is the Galactic latitude of the galaxy; the parameter $C$ in Eq. (1) is given by $C=10^{[0.4 \mathrm{~A}(\mathrm{H} \alpha)]}$. Thus, the $\mathrm{H} \alpha$ luminosity expressed in solar luminosities is given by

$$
L(\mathrm{H} \alpha)=3.13 \times 10^{16} D^{2} f(\mathrm{H} \alpha) C,
$$

where $D$ is in units of $\mathrm{Mpc}, f(\mathrm{H} \alpha)$ is in units of ergs $\mathrm{cm}^{-2} \mathrm{~s}^{-1}$. The resulting $\mathrm{H} \alpha$ luminosities are listed in Table 3.

\subsection{CO Fluxes and Molecular Gas Masses}

Observations of the ${ }^{12} \mathrm{CO} J=1 \rightarrow 0$ emission were made as part of the FCRAO Extragalactic CO Survey using the $14 \mathrm{~m}$ telescope of the Five College Radio Astronomy Observatory between 1980 November and 1992 November. At the frequency of the $\mathrm{CO}$ line $(115.271203 \mathrm{GHz})$, the telescope has a half-power beam width of $45^{\prime \prime}$. The data are presented elsewhere (Young et al. 1995), but a brief description of the relevant details is included here. The $\mathrm{CO}$ observations were made along the major axis of each galaxy at $45^{\prime \prime}$ spacing out to the radius at which the emission fell below $I_{\mathrm{CO}}$ $=1 K\left(T_{A}^{*}\right) \mathrm{km} \mathrm{s}^{-1}$, or if no emission was detected, out to $1 / 2$ of the optical radius. Global CO fluxes were derived by modeling the radial dependence of the $\mathrm{CO}$ emission in each galaxy, and determining the flux of the model which best fit the observations when convolved with a $45^{\prime \prime}$ Gaussian beam. The global flux derived in this way is not very sensitive to the particular $\mathrm{CO}$ distribution assumed.

The uncertainties in the global $\mathrm{CO}$ fluxes are estimated to be $\pm 30 \%$, based on a comparison of major axis maps with fully sampled $\mathrm{CO}$ observations of galaxies (cf. Kenney \& Young 1988). An independent determination of the global CO flux uncertainties, derived from a comparison of the measurements from the FCRAO Extragalactic CO Survey with measurements for the same galaxies from other studies published in the literature, indicates that the global $\mathrm{CO}$ fluxes are accurate to $\pm 40 \%$ (Young et al. 1995). The CO fluxes for the galaxies we have imaged in $\mathrm{H} \alpha$ are listed in Table 3.

The $\mathrm{H}_{2}$ masses were derived from the $\mathrm{CO}$ fluxes using the conversion factor $N\left(\mathrm{H}_{2}\right) / I_{\mathrm{CO}}=2.8 \times 10^{20} \mathrm{H}_{2} \mathrm{~cm}^{-2}\left[K\left(T_{R}\right)\right.$ 
TABLE 3. Global galaxy fluxes, luminosities, and masses.

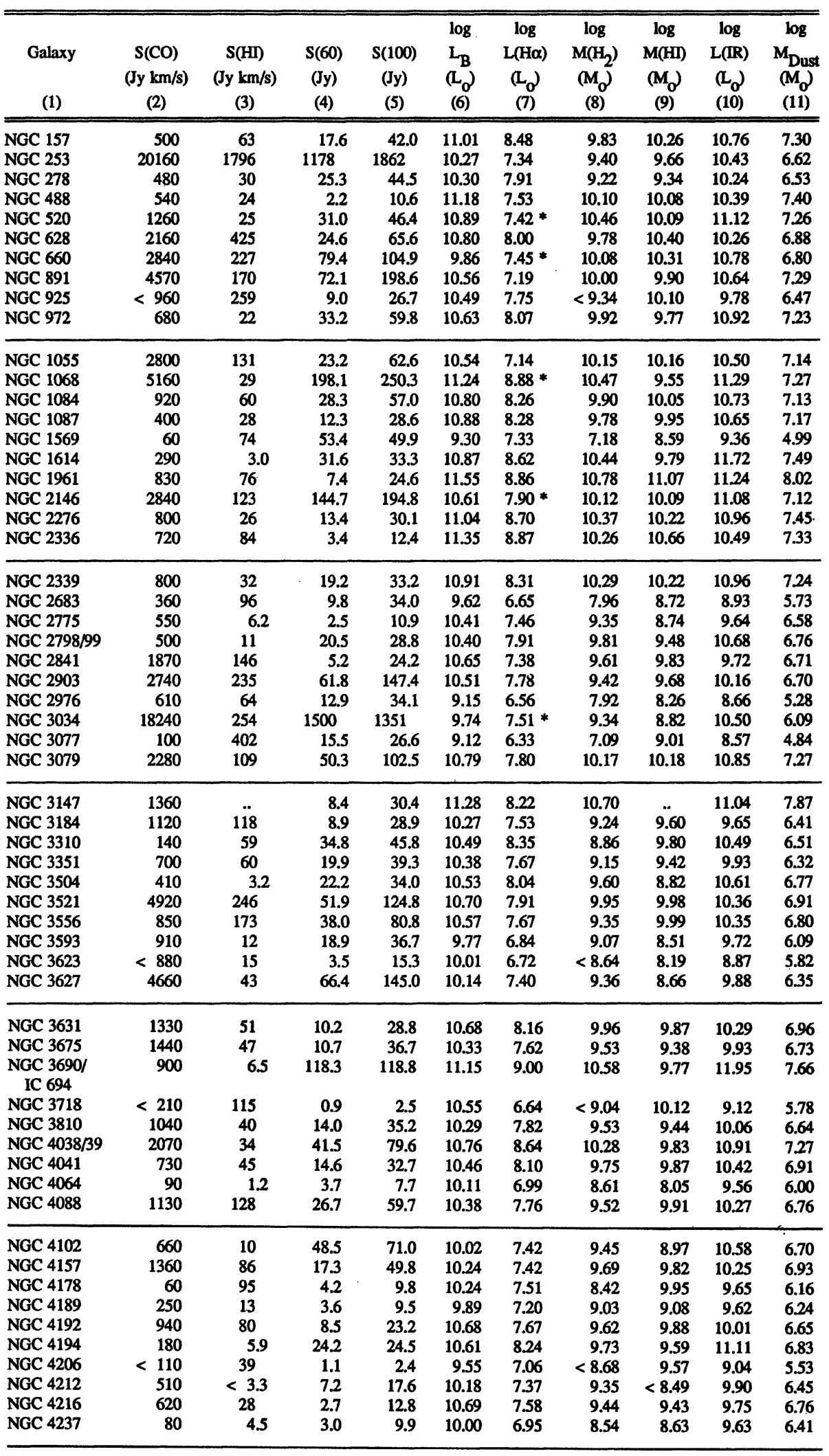


TABLE 3. (continued)

\begin{tabular}{|c|c|c|c|c|c|c|c|c|c|c|}
\hline $\begin{array}{c}\text { Galaxy } \\
\text { (1) }\end{array}$ & $\begin{array}{c}S(C O) \\
(J y k m / s) \\
(2)\end{array}$ & $\begin{array}{c}\text { S(HI) } \\
(\text { Jy } \mathbf{k m} / \mathrm{s}) \\
(3)\end{array}$ & $\begin{array}{c}S(60) \\
(J y) \\
(4)\end{array}$ & $\begin{array}{c}\mathbf{S}(100) \\
(\mathbf{J y}) \\
(5)\end{array}$ & $\begin{array}{c}\log \\
\mathrm{L}_{B} \\
\left(\mathrm{~L}_{0}\right) \\
(6)\end{array}$ & $\begin{array}{c}\log \\
\mathrm{L}(\mathrm{H} \alpha) \\
\left(\mathrm{L}_{0}\right) \\
(7)\end{array}$ & $\begin{array}{c}\log \\
M\left(\mathrm{H}_{2}\right) \\
\left(\mathrm{M}_{0}\right) \\
(8)\end{array}$ & $\begin{array}{c}\log \\
M(H) \\
\left(M_{0}\right) \\
(9)\end{array}$ & $\begin{array}{c}\log \\
\mathrm{L}(\mathrm{IR}) \\
\left(\mathrm{L}_{0}\right) \\
(10) \\
\end{array}$ & $\begin{array}{c}\log \\
M_{\text {Dust }} \\
\left(M_{0}\right) \\
(11)\end{array}$ \\
\hline $\begin{array}{l}\text { NGC } 4254 \\
\text { NGC } 4293 \\
\text { NGC } 4294 \\
\text { NGC } 4298 / \\
\text { NGC } 4302 \\
\text { NGC } 4299 \\
\text { NGC } 4303 \\
\text { NGC } 4321 \\
\text { NGC } 4351 \\
\text { NGC } 4380\end{array}$ & $\begin{array}{r}3000 \\
270 \\
<\quad 35 \\
1280 \\
<\quad 35 \\
2280 \\
3340 \\
<\quad 70 \\
<120\end{array}$ & $\begin{array}{c}12 \\
76 \\
50 \\
3.4 \\
2.5\end{array}$ & $\begin{array}{c}2.7 \\
37.9 \\
25.6 \\
0.79 \\
0.89\end{array}$ & $\begin{array}{r}4.9 \\
78.6 \\
69.2 \\
2.4 \\
3.1\end{array}$ & $\begin{array}{r}10.72 \\
10.49 \\
9.95 \\
10.26 \\
\\
\\
9.73 \\
10.81 \\
10.85 \\
9.81 \\
9.59\end{array}$ & $\begin{array}{l}7.48 \\
8.44 \\
8.01 \\
6.67 \\
6.82\end{array}$ & $\begin{array}{r}10.12 \\
9.07 \\
<8.19 \\
9.75 \\
\\
<8.19 \\
10.00 \\
10.17 \\
<8.48 \\
<8.72\end{array}$ & $\begin{array}{r}9.86 \\
<8.88 \\
9.33 \\
9.56 \\
\\
9.06 \\
9.85 \\
9.67 \\
8.51 \\
8.37\end{array}$ & $\begin{array}{r}10.60 \\
9.72 \\
9.46 \\
10.01 \\
\\
9.39 \\
10.57 \\
10.49 \\
9.02 \\
9.13\end{array}$ & $\begin{array}{l}7.17 \\
6.21 \\
5.84 \\
6.93 \\
\\
5.71 \\
7.00 \\
7.11 \\
5.73 \\
5.94\end{array}$ \\
\hline $\begin{array}{l}\text { NGC } 4394 \\
\text { NGC } 4413 \\
\text { NGC } 4419 \\
\text { NGC } 4424 \\
\text { NGC } 4438 \\
\text { NGC } 4450 \\
\text { NGC } 4459 \\
\text { NGC } 4498 \\
\text { NGC } 4501 \\
\text { NGC } 4519\end{array}$ & $\begin{array}{r}280 \\
<\quad 30 \\
920 \\
60 \\
210 \\
450 \\
<160 \\
<120 \\
2220 \\
<120\end{array}$ & $\begin{array}{c}7 \\
3 \\
2.0 \\
3 \\
11 \\
4 \\
<\quad 3.3 \\
12 \\
30 \\
41\end{array}$ & $\begin{array}{l}0.98 \\
1.2 \\
8.0 \\
3.2 \\
5.0 \\
1.8 \\
1.9 \\
1.3 \\
20.4 \\
4.0\end{array}$ & $\begin{array}{r}4.7 \\
3.4 \\
15.8 \\
5.9 \\
5.9 \\
12.0 \\
8.4 \\
5.4 \\
4.2 \\
43.0 \\
7.1\end{array}$ & $\begin{array}{r}10.19 \\
9.49 \\
10.37 \\
10.06 \\
10.64 \\
10.55 \\
10.37 \\
9.83 \\
10.85 \\
9.98\end{array}$ & $\begin{array}{l}7.13 \\
6.94 \\
7.29 \\
7.00 \\
7.47 \\
6.92 \\
6.71 \\
7.08 \\
7.96 \\
7.36\end{array}$ & $\begin{array}{r}9.09 \\
<8.12 \\
9.61 \\
8.39 \\
8.97 \\
9.30 \\
<8.85 \\
<8.72 \\
9.99 \\
<8.74\end{array}$ & $\begin{array}{r}8.84 \\
8.39 \\
8.28 \\
8.44 \\
9.02 \\
8.62 \\
<8.49 \\
9.05 \\
9.45 \\
9.59\end{array}$ & $\begin{array}{r}9.32 \\
9.18 \\
9.88 \\
9.47 \\
9.74 \\
9.57 \\
9.38 \\
9.26 \\
10.44 \\
9.55\end{array}$ & $\begin{array}{l}6.33 \\
5.86 \\
6.27 \\
5.79 \\
6.27 \\
6.58 \\
6.03 \\
6.01 \\
7.17 \\
5.85\end{array}$ \\
\hline $\begin{array}{l}\text { NGC } 4522 \\
\text { NGC } 4526 \\
\text { NGC } 4527 \\
\text { NGC } 4532 \\
\text { NGC } 4535 \\
\text { NGC } 4536 \\
\text { NGC } 4548 \\
\text { NGC } 4561 \\
\text { NGC } 4567 / \\
\text { NGC } 4568 \\
\text { NGC } 4569\end{array}$ & $\begin{array}{r}<130 \\
<\quad 90 \\
1800 \\
<\quad 60 \\
1570 \\
740 \\
540 \\
<120 \\
1550 \\
1500\end{array}$ & $\begin{array}{c}7.0 \\
31 \\
105 \\
42 \\
73 \\
75 \\
13 \\
25 \\
21 \\
7.0\end{array}$ & $\begin{array}{r}1.5 \\
5.8 \\
33.7 \\
9.6 \\
9.6 \\
12.2 \\
31.6 \\
2.6 \\
1.3 \\
21.2 \\
\\
\\
10.8\end{array}$ & $\begin{array}{r}4.2 \\
15.5 \\
66.2 \\
15.9 \\
33.1 \\
45.8 \\
12.4 \\
3.0 \\
36.6\end{array}$ & $\begin{array}{r}9.60 \\
10.72 \\
10.50 \\
10.09 \\
10.65 \\
10.59 \\
10.51 \\
9.82 \\
10.52 \\
\end{array}$ & $\begin{array}{l}6.81 \\
7.87 \\
7.82 \\
7.75 \\
7.84 \\
7.76 \\
7.03 \\
7.12 \\
7.72\end{array}$ & $\begin{array}{r}<8.76 \\
<8.60 \\
9.90 \\
<8.42 \\
9.84 \\
9.51 \\
9.38 \\
<8.72 \\
9.83 \\
\\
\\
9.82\end{array}$ & $\begin{array}{r}8.82 \\
9.47 \\
10.00 \\
9.60 \\
9.84 \\
9.85 \\
9.08 \\
9.37 \\
9.31\end{array}$ & $\begin{array}{r}9.27 \\
9.84 \\
10.50 \\
9.92 \\
10.16 \\
10.41 \\
9.74 \\
9.13 \\
10.40 \\
\\
\\
10.08\end{array}$ & $\begin{array}{l}5.91 \\
6.46 \\
6.89 \\
6.15 \\
6.80 \\
6.52 \\
6.75 \\
5.65 \\
7.03\end{array}$ \\
\hline $\begin{array}{l}\text { NGC } 4571 \\
\text { NGC } 4579 \\
\text { NGC } 4639 \\
\text { NGC } 4647 \\
\text { NGC } 4651 \\
\text { NGC } 4654 \\
\text { NGC } 4689 \\
\text { NGC } 4710 \\
\text { NGC } 4713 \\
\text { NGC } 4736\end{array}$ & $\begin{array}{r}380 \\
910 \\
<\quad 70 \\
600 \\
350 \\
730 \\
710 \\
200 \\
<\quad 70 \\
2560\end{array}$ & $\begin{array}{c}12 \\
8.1 \\
13 \\
6.6 \\
50 \\
47 \\
7.0 \\
<2.1 \\
41 \\
89\end{array}$ & $\begin{array}{r}1.6 \\
6.0 \\
1.8 \\
5.9 \\
6.2 \\
14.0 \\
3.0 \\
6.2 \\
5.1 \\
73.6\end{array}$ & $\begin{array}{r}6.4 \\
21.1 \\
5.1 \\
16.6 \\
16.8 \\
38.2 \\
10.1 \\
13.0 \\
11.1 \\
135.3\end{array}$ & $\begin{array}{r}9.45 \\
10.66 \\
10.03 \\
10.14 \\
10.40 \\
10.49 \\
9.77 \\
10.29 \\
10.05 \\
10.40\end{array}$ & $\begin{array}{l}7.19 \\
7.61 \\
7.31 \\
7.43 \\
7.48 \\
7.60 \\
7.26 \\
7.71 \\
7.58 \\
7.87\end{array}$ & $\begin{array}{r}9.22 \\
9.60 \\
<8.49 \\
9.42 \\
9.19 \\
9.51 \\
9.49 \\
8.94 \\
<8.49 \\
9.09\end{array}$ & $\begin{array}{r}9.05 \\
8.88 \\
9.10 \\
8.79 \\
9.67 \\
9.65 \\
8.82 \\
<8.30 \\
9.59 \\
8.96\end{array}$ & $\begin{array}{r}9.44 \\
9.96 \\
9.35 \\
9.86 \\
9.87 \\
10.23 \\
9.64 \\
9.79 \\
9.71 \\
9.86\end{array}$ & $\begin{array}{l}6.37 \\
6.77 \\
6.01 \\
6.53 \\
6.51 \\
6.86 \\
6.43 \\
6.23 \\
6.17 \\
6.19\end{array}$ \\
\hline $\begin{array}{l}\text { NGC } 4808 \\
\text { NGC } 4826 \\
\text { NGC } 5005 \\
\text { NGC } 5194 \\
\text { NGC } 5248 \\
\text { NGC } 5631 \\
\text { NGC } 5907 \\
\text { NGC } 6240 \\
\text { NGC } 6643 \\
\text { NGC } 6946\end{array}$ & $\begin{array}{r}<100 \\
2170 \\
1260 \\
9450 \\
1190 \\
\ddot{1230} \\
300 \\
420 \\
12370\end{array}$ & $\begin{array}{c}85 \\
42 \\
19 \\
244 \\
94 \\
7.6 \\
220 \\
.0 \\
39 \\
872\end{array}$ & $\begin{array}{c}7.0 \\
40.0 \\
22.1 \\
128 \\
21.2 \\
\ddot{10.4} \\
22.4 \\
11.5 \\
161.3\end{array}$ & $\begin{array}{c}16.4 \\
77.4 \\
63.9 \\
292 \\
49.3 \\
\ddot{4} .8 \\
26.9 \\
32.9 \\
344.4\end{array}$ & $\begin{array}{r}9.98 \\
10.36 \\
10.78 \\
10.71 \\
10.68 \\
10.63 \\
10.55 \\
11.04 \\
10.84 \\
10.80\end{array}$ & $\begin{array}{c}7.47 \\
7.17 \\
7.78 \\
8.13 \\
8.03 \\
< \\
7.41 \\
9.26 \\
8.20 \\
8.16\end{array}$ & $\begin{array}{r}<8.64 \\
9.13 \\
9.80 \\
9.98 \\
9.80 \\
\ddot{9} .52 \\
10.88 \\
9.75 \\
10.14\end{array}$ & $\begin{array}{r}9.91 \\
8.74 \\
9.32 \\
9.72 \\
10.03 \\
9.58 \\
10.10 \\
\ddot{10.04} \\
10.32\end{array}$ & $\begin{array}{r}9.87 \\
9.72 \\
10.51 \\
10.49 \\
10.43 \\
. \ddot{0} \\
10.08 \\
12.00 \\
10.64 \\
10.61\end{array}$ & $\begin{array}{c}6.40 \\
6.09 \\
7.18 \\
6.99 \\
6.96 \\
.0 \\
7.05 \\
7.92 \\
7.31 \\
7.06\end{array}$ \\
\hline $\begin{array}{l}\text { NGC } 6951 \\
\text { NGC } 7217 \\
\text { NGC } 7331 \\
\text { NGC } 7479 \\
\text { NGC } 7541 \\
\text { NGC } 7625 \\
\text { Arp } 220 \\
\text { IC } 342 \\
\text { IC } 356 \\
\text { Mrk 231 }\end{array}$ & $\begin{array}{r}1440 \\
440 \\
4160 \\
1050 \\
650 \\
270 \\
570 \\
29220 \\
1200 \\
100\end{array}$ & $\begin{array}{r}35 \\
16 \\
177 \\
32 \\
42 \\
18 \\
. \ddot{357} \\
104 \\
<1.5\end{array}$ & $\begin{array}{r}16.4 \\
5.8 \\
41.6 \\
14.4 \\
20.2 \\
9.1 \\
106.6 \\
302.0 \\
6.8 \\
33.3\end{array}$ & \begin{tabular}{r|}
43.6 \\
20.4 \\
10.7 \\
25.8 \\
41.8 \\
17.7 \\
117.8 \\
661.7 \\
28.8 \\
30.8
\end{tabular} & $\begin{array}{l}10.72 \\
10.77 \\
11.08 \\
11.09 \\
10.97 \\
10.35 \\
10.67 \\
10.35 \\
10.67 \\
11.45\end{array}$ & $\begin{array}{l}7.94 \\
7.68 \\
8.14 \\
8.37 \\
8.42 \\
7.90 \\
7.89 * \\
7.01 \\
7.39 \\
9.57\end{array}$ & $\begin{array}{r}10.22 \\
9.46 \\
10.35 \\
10.50 \\
10.37 \\
9.62 \\
10.88 \\
9.81 \\
9.74 \\
10.85\end{array}$ & $\begin{array}{r}9.94 \\
9.35 \\
10.31 \\
10.32 \\
10.51 \\
9.78 \\
. \ddot{10} \\
10.23 \\
10.00 \\
<10.35\end{array}$ & $\begin{array}{l}10.71 \\
10.12 \\
10.79 \\
10.94 \\
11.21 \\
10.47 \\
12.40 \\
10.19 \\
10.11 \\
12.61\end{array}$ & $\begin{array}{l}7.33 \\
6.93 \\
7.44 \\
7.25 \\
7.64 \\
6.85 \\
8.22 \\
6.66 \\
7.04 \\
8.22\end{array}$ \\
\hline
\end{tabular}


Col. (1) Galaxy name-NGC, IC, Mrk, or Arp designation.

Notes to Table 3

Col. (2) Global CO flux, in units of Jy km/sec, from the FCRAO Extragalactic CO Survey (Young et al. 1995). The CO flux is that of the model distribution which best matches the observed distribution of $\mathrm{CO}$ integrated intensities when sampled with a $45^{\prime \prime}$ Gaussian beam. The models are truncated at the radius of the outer edge of the telescope beam for the outermost $\mathrm{CO}$ observation. Corrections for source-beam coupling are applied. Uncertainties arising from incomplete sampling of the disk are typically $\sim 20 \%$. The overall $1 \sigma$ uncertainty in the global CO flux is $\sim 30 \%$. Upper limits are $2 \sigma$.

Col. (3) Global HI flux, in units of Jy km/sec, from the compilation of Huchtmeier et al. (1983). Upper limits are $2 \sigma$.

Col. (4) IRAS flux density at $60 \mu \mathrm{m}$. For the galaxies smaller than 8', the flux densities listed are those derived from one-dimensional coaddition of the IRAS data, or Addscans, using the post-November 1988 calibration. (The November 1988 calibration corrected for a systematic error between the point source and Addscan flux densities, thereby placing the Addscan flux densities on the Point Source Catalogue scale.) For the galaxies larger than $8^{\prime}$, the flux densities listed are based on the IRAS Survey two-dimensional coadded flux densities of Rice et al. (1988). An additional calibration factor of 1.18 has been applied to the $60 \mu \mathrm{m}$ data of Rice et al., since the one-dimensional Addscan flux densities are 1.18 times higher than the two-dimensional Survey coadded flux densities for galaxies between 5' and 8' in size (see Devereux and Young 1990, Appendix). The $1 \sigma$ uncertainty in the flux densities is $\sim 10 \%$.

Col. (5) IRAS flux density at $100 \mu \mathrm{m}$. For the galaxies smaller than $8^{\prime}$, the flux densities listed are those derived from one-dimensional coaddition of the IRAS data, or Addscans, using the post-November 1988 calibration. For the galaxies larger than $8^{\prime}$, the flux densities listed are based on the IRAS Survey two-dimensional coadded flux densities of Rice et al. (1988), with no additional calibration factor since the one- and two-dimensional coadded flux densities agree at $100 \mu \mathrm{m}$ for galaxies between 5' and 8' in size (see Devereux and Young 1990, Appendix). To $1 \sigma$ uncertainty in the flux densities is $\sim 10 \%$.

Col. (6) Logarithm of the blue luminosity in units of $\mathrm{L}_{0}$, derived from the blue magnitude corrected for Galactic and internal absorption, as listed in Table $\mathrm{I}$, and assuming $\mathrm{M}_{\mathrm{B}}(\mathrm{Sun})=+5.48$.

Col. (7) Logarithm of the $\mathrm{H} \alpha$ luminosity in units of $\mathrm{L}_{0}$, corrected for foreground Galactic extinction. The luminosity is derived from the distance D and the extinction-corrected $\mathrm{H} \alpha$ flux $[\mathrm{f}(\mathrm{H} \alpha)]$, where $\mathrm{L}(\mathrm{H} \alpha)=4 \pi \mathrm{D}^{2} \mathrm{f}(\mathrm{H} \alpha)$. The Galactic extinction correction in magnitudes is of the form $\mathrm{A}(\mathrm{mag})=0.08(\mathrm{csc}|\mathrm{b}|-1)$, where $\mathrm{b}$ is the Galactic latitude of each galaxy. The values listed include no corrections for extinction internal to the galaxies in question. Galaxies indicated by an asterisk are those for which internal extinction corrections have been determined from [SIII] imaging (Young, Kleinmann, and Allen 1988). The corrected $\mathrm{H} \alpha$ luminosities for these galaxies, in the same units as the values in column 7 of this Table, are: NGC 520 (8.83), NGC 660 (9.32), NGC 1068 (9.12), NGC 2146 (8.72), NGC 3034 (8.12), and Arp 220 (9.68).

Col. (8) Logarithm of the global molecular gas mass in units of $\mathrm{M}_{0}$, computed from the global CO flux [S(CO)] from Col. (2), and assuming a CO $\rightarrow \mathrm{H}_{2}$ conversion constant of $\mathrm{N}\left(\mathrm{H}_{2}\right) / \mathrm{I}(\mathrm{CO})=2.8 \times 10^{20} \mathrm{H}_{2} / \mathrm{cm}^{2} /\left[\mathrm{K}\left(\mathrm{T}_{\mathrm{R}}\right) \mathrm{km} / \mathrm{sec}\right]$ (Bloemen et al. 1986). For this value of the conversion factor, the $\mathrm{H}_{2}$ masses are given by $\mathrm{M}\left(\mathrm{H}_{2}\right)=1.1 \times 10^{4} \mathrm{D}^{2} \mathrm{~S}(\mathrm{CO})$, where $\mathrm{D}$ is the distance in $\mathrm{Mpc}$. Upper limits are $2 \sigma$.

Col. (9) Logarithm of the atomic gas mass in units of $\mathrm{M}_{0}$, computed from the global $\mathrm{HI}$ flux in Col. (3). The HI mass is given by $\mathrm{M}(\mathrm{HI}) 2.36 \times 10^{5}$ $\mathrm{D}^{2} \mathrm{~S}(\mathrm{HI})$, where $\mathrm{S}(\mathrm{HI})$ is the $\mathrm{HI}$ flux in Jy $\mathrm{km} / \mathrm{sec}$, and $\mathrm{D}$ is the distance in Mpc. Upper limits are $2 \sigma$.

Col. (10) Logarithm of the far-infrared luminosity from $\sim 1$ to $500 \mu \mathrm{m}$ in units of $L_{0}$, computed from the IRAS 60 and $100 \mu \mathrm{m}$ flux densities. The IR luminosity is given by $\mathrm{L}(\mathrm{IR})=3.75 \times 10^{5} \mathrm{D}^{2} \mathrm{C}[2.58 \mathrm{~S}(60)+\mathrm{S}(100)]$, where $\mathrm{D}$ is the distance in $\mathrm{Mpc}, \mathrm{S}(60)$ and $\mathrm{S}(100)$ are the IRAS flux densities listed in Columns (4) and (5) in Jy. The constant $C$ corrects for the flux missed shortward of $40 \mu \mathrm{m}$ and longward of $120 \mu \mathrm{m}$, and is a function of the $\mathrm{S}(60) / \mathrm{S}(100)$ ratio; the values of C are given in Table B.1 of Catalogued Galaxies in the IRAS Survey (1985). The $1 \sigma$ uncertainty in the luminosities is $\sim 10 \%$.

Col. (11) Logarithm of the warm dust mass in units of $\mathrm{M}_{\mathrm{o}}$, computed from the $100 \mu \mathrm{m}$ flux density, the dust temperature ( $\left.\mathrm{T}_{\text {dust }}\right)$ implied by the ratio of 60 to $100 \mu \mathrm{m}$ flux densities, and the grain properties (size, density, and emissivity) given by Hildebrand (1983). The mass of warm dust emitting at $100 \mu \mathrm{m}$ is given by $M_{\text {dust }}=4.78 \mathrm{~S}(100) \mathrm{D}^{2}\left[\exp \left(143.88 / \mathrm{T}_{\text {dust }}\right)-1\right]$, where $\mathrm{T}_{\text {dust }}$ is in ${ }^{\circ} \mathrm{K}$ and $\mathrm{D}$ is in $\mathrm{Mpc}$.

$\left.\mathrm{km} \mathrm{s}^{-1}\right]^{-1}$ (Bloemen et al. 1986). Kenney \& Young (1989) show that this value of the conversion factor leads to $\mathrm{H}_{2}$ masses in solar units given by

$$
M\left(\mathrm{H}_{2}\right)=1.1 \times 10^{4} D^{2} S(\mathrm{CO}),
$$

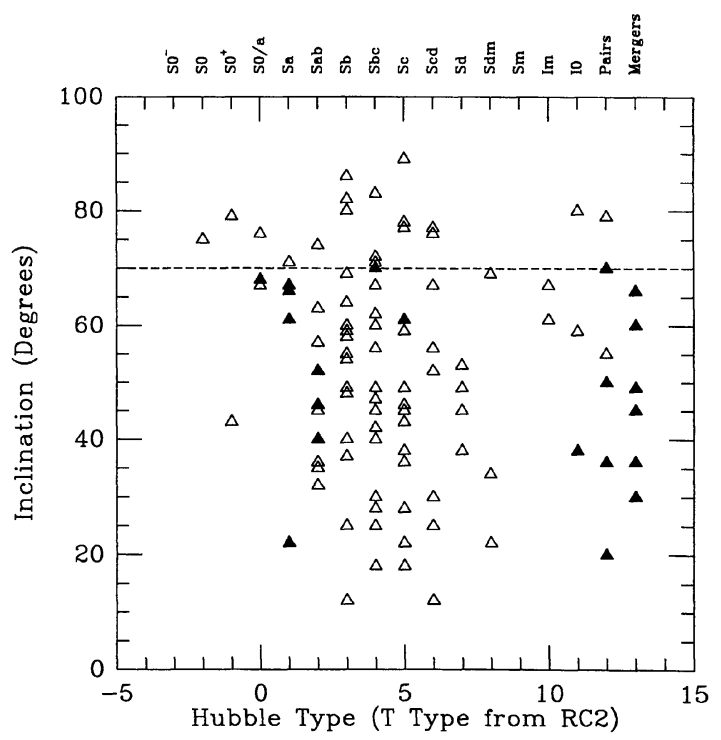

FIG. 3. Galaxy inclinations, plotted as a function of morphological type, for the 120 galaxies in the $\mathrm{H} \alpha$ imaging survey. Solid symbols indicate the galaxies classified as peculiar. The inclinations, listed in Table 4, were derived following Roberts (1969) -also see note to column 4 in Table 4. where $D$ is the distance in $\mathrm{Mpc}$ and $S(\mathrm{CO})$ is the $\mathrm{CO}$ flux in Jy $\mathrm{km} \mathrm{s}^{-1}$.

There has been much discussion in the literature of the accuracy of molecular gas masses for galaxies. Of interest here are the global $\mathrm{H}_{2}$ masses in luminous spiral galaxies.

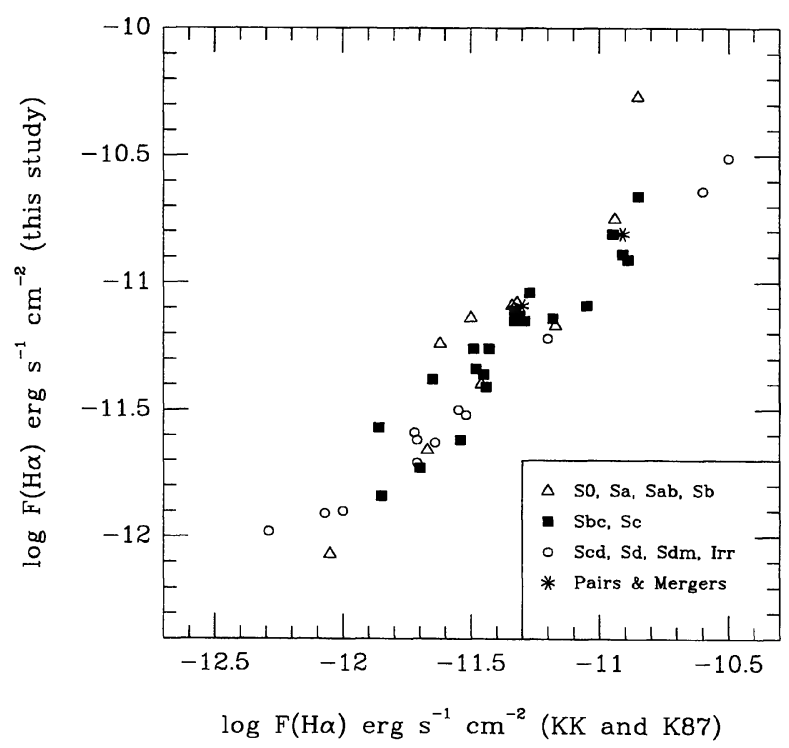

FIG. 4. Comparison of $\mathrm{H} \alpha$ fluxes for 45 galaxies in the present study which overlap with the galaxies studied by Kennicutt \& Kent (1983) and Kennicutt et al. (1987). Galaxies are coded by morphological type as indicated. 
With regard to the absolute accuracy of the Milky Way $\mathrm{CO} \rightarrow \mathrm{H}_{2}$ conversion factor when applied to luminous spiral galaxies, Young \& Scoville (1991; cf. Fig. 1) have shown that the molecular clouds which have been spatially resolved in M31 (Vogel et al. 1987) and M33 (Wilson \& Scoville 1990) have similar ratios of CO luminosity to virial mass as clouds in the inner and outer Milky Way (Scoville et al. 1987; Solomon et al. 1987). This implies that the $\mathrm{CO} \rightarrow \mathrm{H}_{2}$ conversion factor for the Milky Way also applies to the molecular clouds in the Sb galaxy M31 and the Scd galaxy M33.

In this paper, we adopt $\pm 30 \%$ as the uncertainty in the global $\mathrm{H}_{2}$ masses for luminous spiral galaxies. The molecular gas masses for the low metallicity irregular galaxies may be considerably more uncertain (cf. Maloney \& Black 1988). The global molecular gas masses for the galaxies in this sample are listed in Table 3.

\subsection{H I Fluxes and Atomic Gas Masses}

$\mathrm{H}$ I fluxes for the galaxies in our sample have been taken from the compilation of Huchtmeier et al. $(1983,1988)$, provided that the measurement refers to the global $\mathrm{H}$ I flux of the galaxy. The specific references for the $\mathrm{H}$ I measurements are listed elsewhere (Young et al., in preparation). The mass of atomic hydrogen in solar masses is given by

$$
M(\mathrm{H} \mathrm{I})=2.36 \times 10^{5} D^{2} S(\mathrm{H} \mathrm{I}),
$$

where $D$ is the distance in $\mathrm{Mpc}$ and $S(\mathrm{HI})$ is the $\mathrm{HI}$ flux in $\mathrm{Jy} \mathrm{km} \mathrm{s}^{-1}$. The global $\mathrm{H}$ I fluxes and atomic gas masses are listed in Table 3. The uncertainties in these values are of order 30\% (cf. Huchtmeier et al. 1983, 1988), similar to the uncertainties in the molecular gas masses.

\subsection{IRAS Flux Densities, Far-IR Luminosities, and Warm Dust Masses}

The infrared luminosities used here are based on coadded IRAS flux densities at 60 and $100 \mu \mathrm{m}$ using the postNovember 1988 calibration. For galaxies smaller than $8^{\prime}$ in diameter, we have used the one-dimensional coadded IRAS data, or addscans to determine the flux densities. For galaxies $8^{\prime}$ in diameter and larger, we have used the two-dimensional coadded IRAS Survey flux densities reported by Rice et al. (1988). An additional calibration factor of 1.18 has been applied to the $60 \mu \mathrm{m}$ data of Rice $e t$ al., since one-dimensional Addscan flux densities are 1.18 times higher than the twodimensional Survey coadded flux densities for galaxies between $5^{\prime}$ and $8^{\prime}$ in diameter (see Young et al. 1989; Devereux \& Young 1990b, Appendix). The IRAS 60 and 100 $\mu \mathrm{m}$ flux densities for the galaxies in the present sample are given in Table 3.

The far-IR luminosities from $\sim 1$ to $500 \mu \mathrm{m}$ have been derived using the flux densities determined from the IRAS data. The far-IR luminosity is given by Lonsdale et al. (1985) as

$$
\begin{aligned}
L(\mathrm{IR})= & 4 \pi D^{2}\left[1 . 2 6 C \left\{2.58 \times 10^{-14} S(60)+1.0\right.\right. \\
& \left.\left.\times 10^{-14} S(100)\right\}\right],
\end{aligned}
$$

where $D$ is the distance, the factor 1.26 corrects for the gap between the 60 and $100 \mu \mathrm{m}$ bandpasses and the filter response, and the constant $C$ is a correction factor for the flux longward of $120 \mu \mathrm{m}$ and shortward of $40 \mu \mathrm{m}$. The values of $C$ are a function of the $S(60) / S(100)$ ratio; they are taken from Table B.1 of Catalogued Galaxies in the IRAS Survey (1985). In solar units, Eq. (5) becomes

$$
L(\mathrm{IR})=3.75 \times 10^{5} D^{2} C[2.58 S(60)+S(100)],
$$

where $D$ is in Mpc, $S(60)$ and $S(100)$ are in Jy as listed in Table 3. The far-IR luminosities are presented in Table 3.

We have also used the IRAS flux densities to estimate the mass of warm dust in each galaxy. Following the analysis of Hildebrand (1983) and assuming a single temperature component, the mass of warm dust is given by

$$
\mathscr{M}_{\text {Dust }}=(4 / 3) a \rho Q_{100}\left[S_{100} D^{2} / B(100, T)\right],
$$

where $a$ is the weighted grain size, $\rho$ is the grain density, $Q_{100}$ is the $100 \mu \mathrm{m}$ grain emissivity, $S_{100}$ is the flux density at $100 \mu \mathrm{m}$, and $B(100, T)$ is the intensity of the blackbody of temperature $T$ at $100 \mu \mathrm{m}$. Using the grain size, density, and emissivity given by Hildebrand (1983), (4/3) $a \rho Q_{100}=0.04$ $\mathrm{g} \mathrm{cm}^{-2}$. The mass of $100 \mu \mathrm{m}$ emitting dust then becomes

$$
\mathscr{M}_{\text {Dust }}=4.78 S(100) D^{2}\left[\exp \left(143.88 / T_{\text {dust }}\right)^{-1}\right] \text {, }
$$

where the dust mass is in solar masses, $S(100)$ is in Jy, $D$ is the distance in $\mathrm{Mpc}$, and $T_{\text {dust }}$ is the warm dust temperature in K. As discussed in Young et al. $(1986 \mathrm{~b}, 1989)$ and Devereux \& Young (1990b), IRAS is sensitive to warm dust with temperatures $\geqslant 25 \mathrm{~K}$ but not to colder dust with temperatures $\leqslant 20 \mathrm{~K}$ emitting predominantly at wavelengths beyond $100 \mu \mathrm{m}$. Thus, the dust mass we derive will be referred to as the "warm dust mass." These dust masses are listed in Table 3.

\section{STAR FORMATION IN GALAXIES}

\subsection{Global Ho Luminosities and Surface Brightnesses}

The $\mathrm{H} \alpha$ luminosities we derive span more than three orders of magnitude, as do the optical areas of the galaxies. If all galaxies produced the same $\mathrm{H} \alpha$ luminosity per unit surface area, the $\mathrm{H} \alpha$ luminosities would scale linearly with optical area, in the absence of extinction. Figure 5(a) shows the $\mathrm{H} \alpha$ luminosities for the galaxies in our sample plotted versus the face-on optical areas. There is considerable scatter in the $\mathrm{H} \alpha$ luminosities of galaxies of a given surface area, although in general the larger galaxies are more luminous. The solid triangles represent the 22 galaxies whose type classifications include the designation of peculiar (see Table 1). The dashed line represents the mean $\mathrm{H} \alpha$ surface brightness for all of the galaxies in the sample. The data in Fig. 5 indicate that peculiar galaxies exhibit the full range of $\mathrm{H} \alpha$ surface brightness found among the more normal galaxies.

The $\mathrm{H} \alpha$ luminosities versus optical areas are also shown in Fig. 5(b), this time with the highly inclined galaxies indicated. In this figure, the dashed line is a fit to the galaxies with inclinations less than $70^{\circ}$, while the dotted line represents a fit to the galaxies with inclinations greater than $70^{\circ}$. The difference in these two fits indicates that the more face-on galaxies have $\mathrm{H} \alpha$ surface brightnesses $\sim 3$ times 

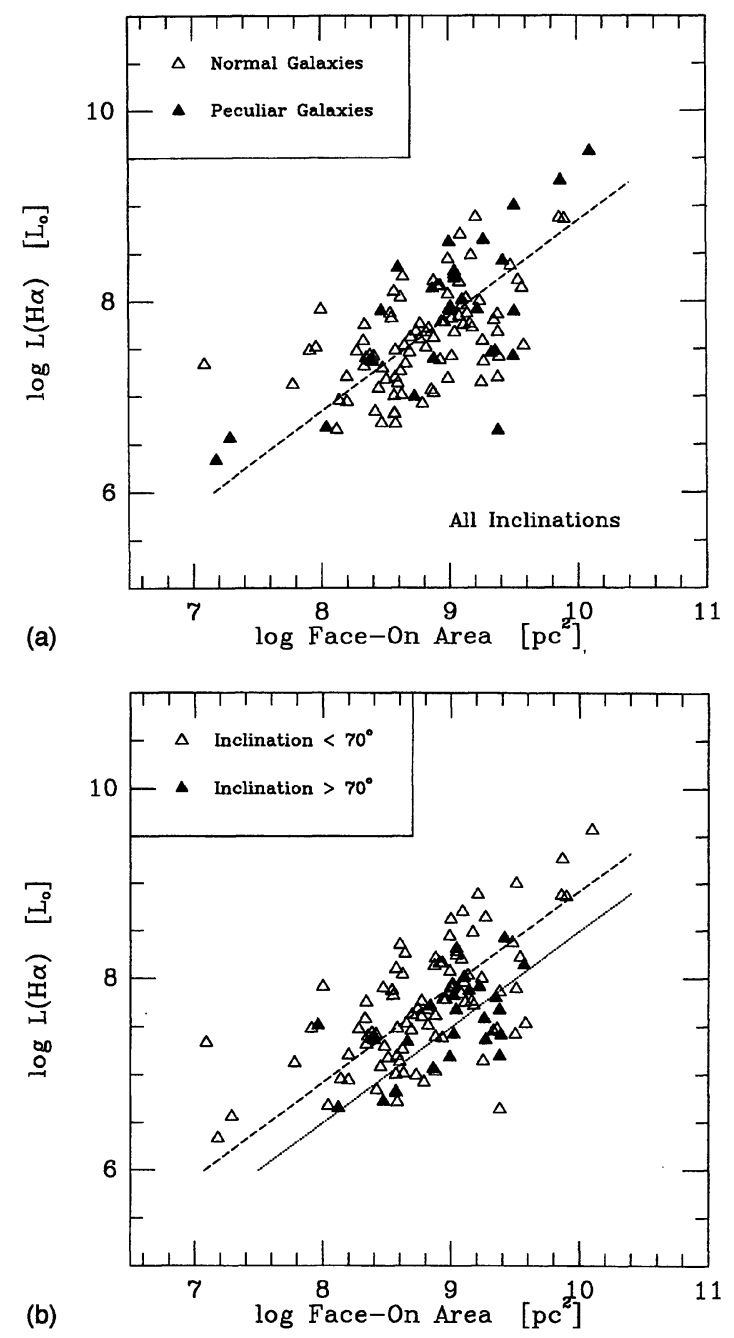

FIG. 5. H $\alpha$ luminosities for the galaxies in the imaging survey, plotted versus the face-on area of the galaxy. Galaxy areas were derived from $D_{25}$ given in Table 1 (from RC2). The solid symbols represent galaxies which are classified as peculiar (see Table 1). The dashed line represents the mean $\mathrm{H} \alpha$ surface brightness for all of the galaxies in the sample. (b) Same as (a), where the solid symbols represent galaxies with inclinations greater than $70^{\circ}$, while open symbols represent the more face-on galaxies. The dotted line represents the mean $\mathrm{H} \alpha$ surface brightness of the highly inclined galaxies, while the dashed line represents the mean $\mathrm{H} \alpha$ surface brightness of the less inclined galaxies. The more face-on galaxies have a mean $\mathrm{H} \alpha$ surface brightness which is a factor of 3 times higher than that of the highly inclined galaxies. The highly inclined galaxy (filled triangle) with the highest $\mathrm{H} \alpha$ surface brightness represents the edge-on galaxy M82.

greater in the mean than the highly inclined galaxies. Therefore, some of the scatter in Fig. 5 is related to extinction, some is related to measurement uncertainties, and some is related to real variations in the $\mathrm{H} \alpha$ surface brightnesses among galaxies.

In order to determine the approximate galaxy inclination above which the extinction causes a significant reduction in the $\mathrm{H} \alpha$ luminosity, we show in Fig. 6 the $\mathrm{H} \alpha$ luminosity per unit face-on surface area, or the $\mathrm{H} \alpha$ surface brightness, as a function of the galaxy inclination. Although there is a large scatter in the observed $\mathrm{H} \alpha$ surface brightnesses of galaxies at each inclination, there is a trend of decreasing $\mathrm{H} \alpha$ surface

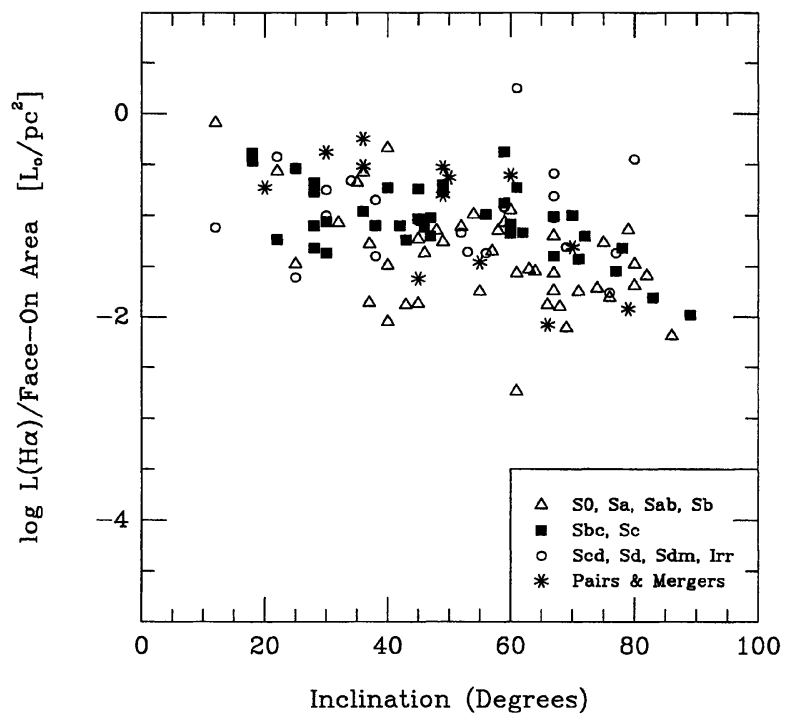

FIG. 6. $\mathrm{H} \alpha$ surface brightnesses plotted as a function of the galaxy inclination. The inclinations, listed in Table 4, were derived following Roberts (1969). Galaxies are coded by morphological type as indicated. Note the trend of decreasing $\mathrm{H} \alpha$ surface brightness with increasing inclination.

brightness for the highly inclined galaxies, with the most pronounced effect for galaxies with inclinations greater than $70^{\circ}$. In the discussion which follows, galaxies with inclinations greater than $70^{\circ}$ are referred to as the "highly inclined" galaxies.

In order to investigate the intrinsic variations in the $\mathrm{H} \alpha$ surface brightnesses among galaxies, it is necessary to exclude the highly inclined galaxies. In Fig. 7(a), we show the $\mathrm{H} \alpha$ luminosities versus the face-on optical areas of the 96 systems with inclinations less than $70^{\circ}$. The dashed line in the figure represents the fit to the $\mathrm{H} \alpha$ surface brightnesses of these galaxies; the galaxies classified as peculiar are indicated with filled symbols. We find no significant difference in the $\mathrm{H} \alpha$ surface brightnesses of the peculiar galaxies when compared with the more normal galaxies in the sample.

In order to investigate the morphological type dependence of the $\mathrm{H} \alpha$ surface brightnesses, in Fig. 7(b) we show the $\mathrm{H} \alpha$ luminosities versus face-on optical areas as in Fig. 7(a), where the data are now coded by galaxy type. Each panel of Fig. 7(b) displays the data for the 96 galaxies with $i<70^{\circ}$; the dotted line represents the fit for all 96 galaxies, the large symbols represent the type indicated, and the small dots represent the remaining galaxies in the sample. Although there is considerable scatter in the observed $\mathrm{H} \alpha$ surface brightnesses within each type, it is clear that the early-type spiral galaxies tend to have lower than average $\mathrm{H} \alpha$ surface brightnesses, while the latest-type spirals and the interacting systems have higher than average $\mathrm{H} \alpha$ surface brightnesses. In the mean, the $\mathrm{H} \alpha$ luminosity per unit face-on surface area is a factor of 2.6 times lower among the early-type spiral galaxies in our sample relative to the later-type spirals and interacting systems.

We have compared the $\mathrm{H} \alpha$ surface brightnesses along the Hubble sequence with the UV surface brightnesses found by Deharveng et al. (1994), and both studies agree that the Sd-Ir 

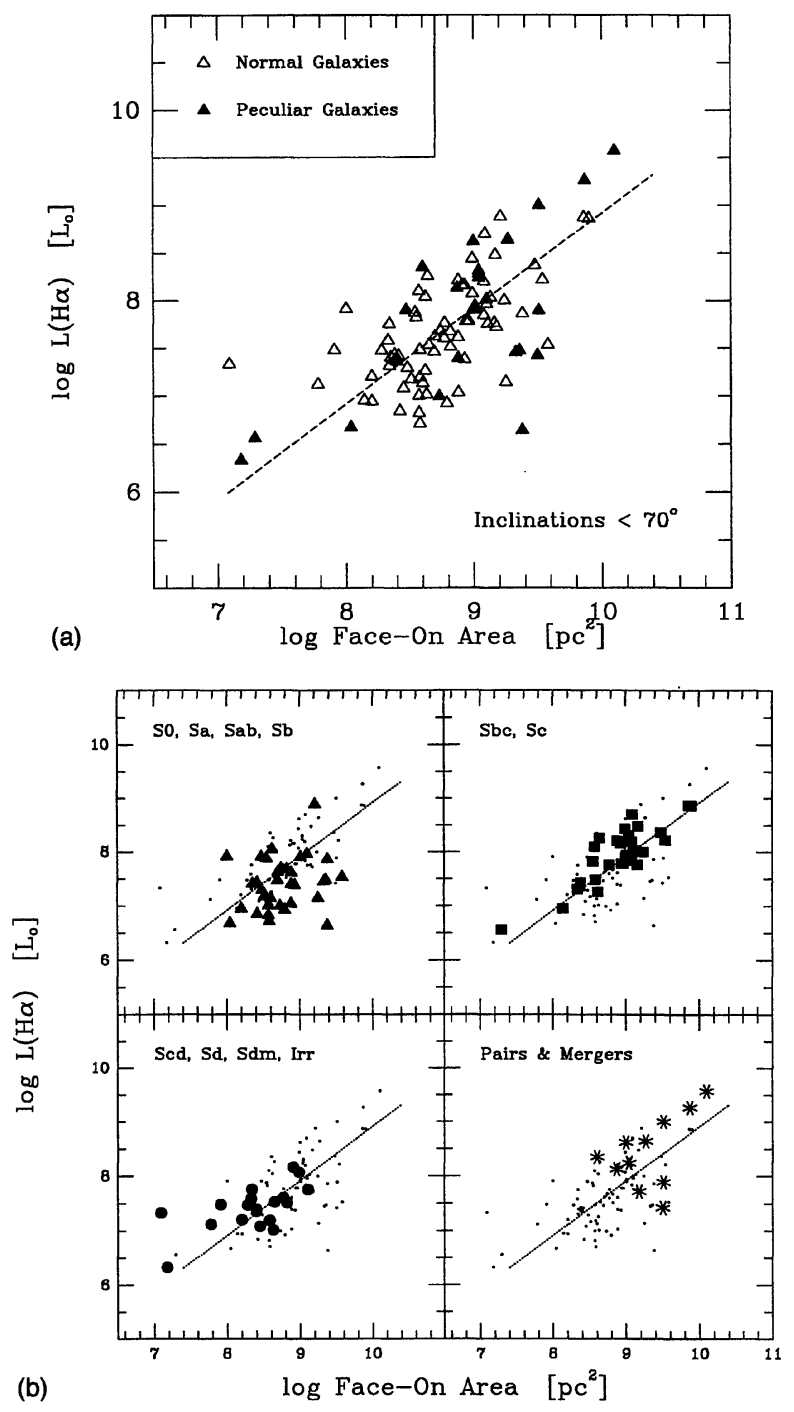

galaxies have $\mathrm{H} \alpha$ and UV surface brightnesses about 1.3-1.4 times higher than for Sbc-Scd galaxies, and about 3 times higher than for $\mathrm{Sa}-\mathrm{Sb}$ galaxies, respectively. These mean values are sample dependent, as Deharveng et al. noted; when we exclude the peculiar galaxies in our sample, the difference in the mean $\mathrm{H} \alpha$ surface brightness between the Sd-Ir and $\mathrm{Sa}-\mathrm{Sb}$ types decreases from a factor of 3 to a factor of 2.5. In this work, the $\mathrm{H} \alpha$ surface brightness variation with morphology is significant at about the $3-4 \sigma$ level. Thus, there is a small difference in the mean $\mathrm{H} \alpha$ surface brightness as a function of type for spiral galaxies along the Hubble sequence, such that the late-type galaxies have a mean surface brightness about a factor of 2-3 times higher than the early types.

Histograms of the $\mathrm{H} \alpha$ surface brightnesses as a function of type are shown in Fig. 7(c). Each panel illustrates the $\mathrm{H} \alpha$ surface brightnesses for the entire sample (dotted line) along with the surface brightnesses within each type category (hatched area) and the mean value for that type (solid dot). From this figure, it is clear that each type exhibits a broad range of surface brightnesses, and that the change in the

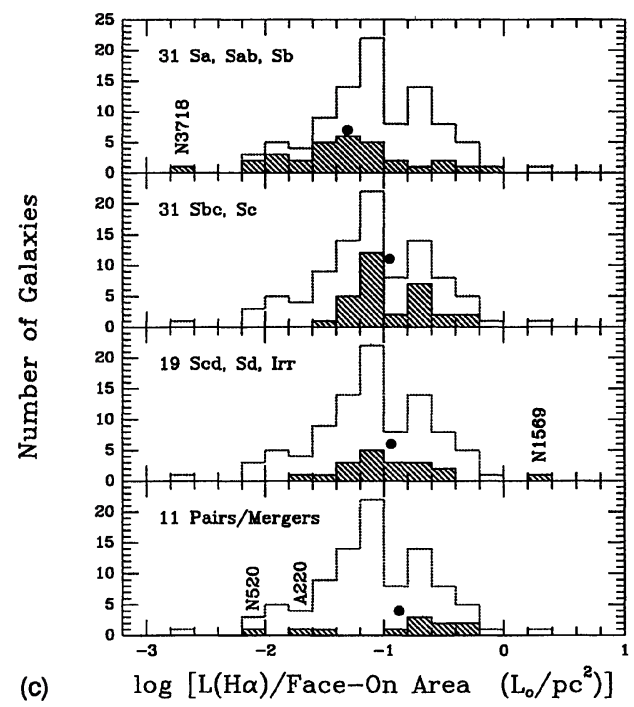

FIG. 7. (a) $\mathrm{H} \alpha$ luminosities for the 96 galaxies in the imaging survey with inclinations $<70^{\circ}$, plotted vs the face-on area of the galaxy. Galaxy areas were derived from $D_{25}$ given in Table 1 (from RC2). Galaxies are coded as peculiar according to Table 1 . The dashed line represents the mean $\mathrm{H} \alpha$ surface brightness for these 96 less inclined galaxies. (b) $\mathrm{H} \alpha$ luminosities versus the face-on areas of the galaxies with inclinations $<70^{\circ}$ for four distinct morphological categories. The large symbols in each panel represent the type indicated, while the small dots represent the remainder of the galaxies with $i<70^{\circ}$. The dotted line in each panel represents the mean $\mathrm{H} \alpha$ surface brightness for the 96 galaxies with $i<70^{\circ}$, as in 7(a). (c) $\mathrm{H} \alpha$ surface brightnesses for galaxies with inclinations $<70^{\circ}$ for four distinct morphological categories. The dotted histogram represents the distribution of surface brightnesses for all of the galaxies with inclinations $<70^{\circ}$, the hatched histogram represents the $\mathrm{H} \alpha$ surface brightnesses for the type indicated, and the solid dot represents the mean value for that type. Several galaxies with extreme values are indicated.

mean $\mathrm{H} \alpha$ surface brightness with type arises primarily from some early-type spiral galaxies with very low surface brightnesses.

This difference in $\mathrm{H} \alpha$ surface brightness as a function of type has led to the understanding that early-type spiral galaxies have less star formation than the later types (cf. Kennicutt 1983; Sandage 1986; Deharveng et al. 1994). We note, however, that the early-type spiral galaxies are by no means lacking in star formation. In fact, there are numerous Sa galaxies with higher $\mathrm{H} \alpha$ luminosities than Sc galaxies of the same size. One reason this $\mathrm{H} \alpha$ emission may have been overlooked in the past is that the highest $\mathrm{H} \alpha$ surface brightnesses are often found in the central region where the overall light distribution is dominated by the nuclear bulge (as in NGC $660,2146,3351,3504$, and 4826 , for example). Whereas photographic studies may have missed the star formation in the central regions of galaxies, the advent of CCD imaging systems has now made the separation of the disk and bulge feasible, revealing the $\mathrm{H}$ II regions which were previously hidden from view. 


\subsection{Global High-Mass Star Formation Rates from $\mathrm{H \alpha}$ Luminosities}

The use of integrated Balmer line luminosities as an indicator of the current star forming activity in galaxies has been discussed extensively in the literature. The underlying assumption which is necessary in order to relate the $\mathrm{H} \alpha$ luminosity to the rate of formation of high mass stars is the form of the initial mass function (IMF). Kennicutt (1983) shows that for an adopted IMF where $\psi(m)$ is proportional to $m^{-1.4}$ for $0.1 \leqslant m \leqslant 1 \mathscr{C}_{0}$ and $m^{-2.5}$ for $1<m \leqslant 100 \mathscr{C}_{0}$, the relationship between the star formation rate (SFR) and the $\mathrm{H} \alpha$ luminosity reduces to a simple expression. The SFR for stars over $10 \mathscr{M}_{0}$, and the total SFR, respectively, are then given by

$$
\begin{aligned}
& \operatorname{SFR}\left(\geqslant 10 \mathscr{M}_{0}\right)=5.45 \times 10^{-9} L(\mathrm{H} \alpha)\left[\mathscr{M}_{0} / \mathrm{yr}\right], \\
& \operatorname{SFR}(\text { total })=3.42 \times 10^{-8} L(\mathrm{H} \alpha)\left[\mathscr{C}_{0} / \mathrm{yr}\right],
\end{aligned}
$$

for $L(\mathrm{H} \alpha)$ in units of solar luminosities. For the $\mathrm{H} \alpha$ luminosities of the galaxies in this study, which range from $10^{6}$ to $10^{10} L_{0}$, the SFR for stars greater than $10 \mathscr{M}_{0}$ is between $5 \times 10^{-3}$ and $50 \mathscr{C}_{0} \mathrm{yr}^{-1}$, while the total SFR for stars with masses from 0.1 to $100 \mathscr{M}_{0}$ is estimated to be between 0.03 and $300 \mathscr{M}_{0} \mathrm{yr}^{-1}$.

\subsection{Comparison of $\mathrm{H} \alpha$ and IR Luminosities}

There is little question that the observed $\mathrm{H} \alpha$ luminosity in spiral galaxies is a consequence of the ionization of the interstellar medium by the UV radiation which is produced by the young high mass stars, and is an indication of the high mass star formation rate. While the initial purpose of our study was to determine the distribution of star formation within spiral galaxies, one important use of the $\mathrm{H} \alpha$ luminosities we derive is to test the extent to which the IR luminosity for the same galaxies also traces the rate of high mass star formation.

There has been much discussion recently of the farinfrared luminosity as a tracer of the rate of high mass star formation in galaxies. Numerous authors have argued that high mass ionizing stars provide the energy which heats the dust in galaxies (Telesco \& Harper 1980; Lonsdale et al. 1985; Young et al. 1984, 1986, 1989; Sanders \& Mirabel 1985; Helou et al. 1985; De Jong et al. 1985; Gavazzi et al. 1986; Sanders et al. 1986, 1987; Solomon \& Sage 1988; Tinney et al. 1990; Devereux \& Young 1990a), while others have argued that low mass nonionizing stars are also important for heating the dust in galaxies (De Jong 1984; Lonsdale \& Helou 1987; Bothun et al. 1989; Rand, et al. 1992; Smith et al. 1994). The critical question is, of course, whether the IR luminosities measured by IRAS can be used to trace the high mass star formation rates for thousands of galaxies.

In Fig. 8, we compare the $\mathrm{H} \alpha$ luminosity with the IR luminosity for the 120 galaxies in our sample, with the galaxies coded by morphological type. The diagonal lines in Fig. 8 represent the ratios of ionizing flux to bolometric luminosity expected for ionizing stars ranging in spectral type from $\mathrm{O} 5$ to B5. For this sample of galaxies, the $\mathrm{H} \alpha$ and IR luminosities are consistent with the values of $L(\mathrm{H} \alpha) / L(\mathrm{IR})$ which would be produced by OB stars (Panagia 1973; De-

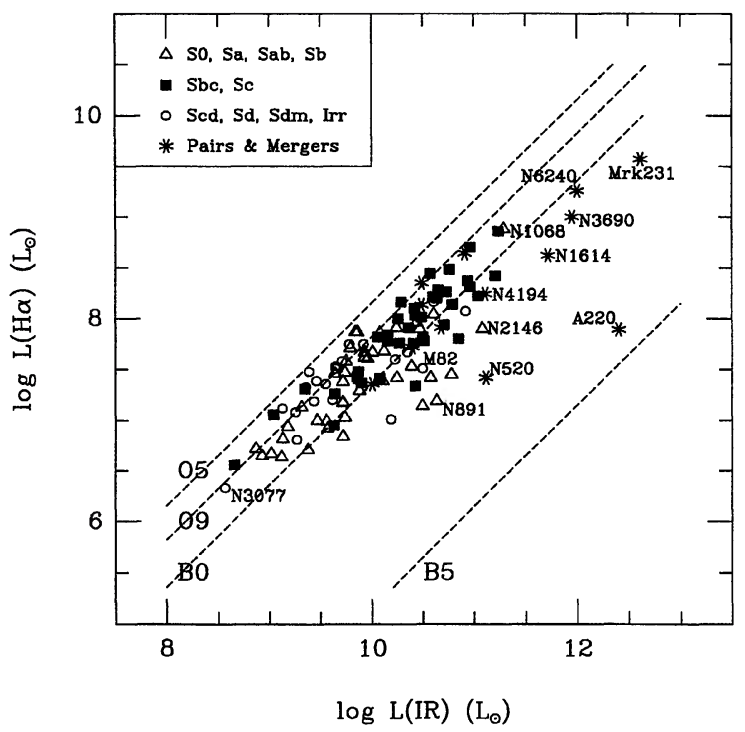

FIG. 8. Comparison of the $\mathrm{H} \alpha$ and far-infrared luminosities for the galaxies in the present study, with all inclinations included. Galaxies are coded by type as indicated. The diagonal dashed lines represent the expected relation between the $\mathrm{H} \alpha$ and bolometric luminosity for O5 $\left(40 \mathscr{L}_{0}\right)$, O9 $\left(20 \mathscr{M}_{0}\right)$, B0 $\left(16 \mathscr{M}_{0}\right)$, and B5 $\left(6 \mathscr{M}_{0}\right)$ stars, following Panagia (1973), as presented by Devereux \& Young (1990a). The lowest values of the ratio of $\mathrm{H} \alpha$ luminosity to far-IR luminosity are found in the systems which suffer the most severe extinction-the edge-on galaxy NGC 891, and the galaxies with obvious dust lanes near the nucleus, NGC 520 and Arp 220.

vereux \& Young 1990a) assuming that photons shortward of the Lyman limit ionize the hydrogen while the bolometric luminosity of the star is radiated in the infrared (WynnWilliams \& Becklin 1974).

One important result illustrated in Fig. 8 is that there is no significant difference in the regions where the early- and late-type spiral galaxies are located. This same point has been made by Devereux \& Young (1990a) from a comparison of the $\mathrm{H} \alpha$ and IR luminosities for the spiral galaxies in the study of Kennicutt \& Kent (1983). If the low mass, nonionizing stars were primarily responsible for heating the dust which is radiating at 60 and $100 \mu \mathrm{m}$, then one would expect the galaxies with large bulges (and large numbers of lowmass stars) to have higher IR luminosities for a given amount of disk star formation than the galaxies without bulges. This is not observed to be the case.

What is perhaps most striking from Fig. 8 is that the observed ratios of $L(\mathrm{H} \alpha) / L(\mathrm{IR})$ are remarkably similar from galaxy to galaxy, in spite of the wide range of properties and diversity of environments among the galaxies included in our study. This is further illustrated in Fig. 9, which is a histogram of the ratio of $\mathrm{H} \alpha$ to far-infrared luminosity for all of the galaxies in the sample. The solid portion of the histogram represents the galaxies with the greatest reddening-those with inclinations greater than $70^{\circ}$, and four merger candidates with obscured nuclei (NGC 520, NGC 660, NGC 2146, and Arp 220). The dashed lines near the top of Fig. 9 represent the ratios of $\mathrm{H} \alpha$ to far-IR luminosity for stars of the stellar spectral types indicated. It appears that the peak of the histogram of $L(\mathrm{H} \alpha) / L(\mathrm{IR})$ values corresponds to stars of spectral type O9-B0, or masses between 16 and $20 \mathscr{C}_{0}$. 


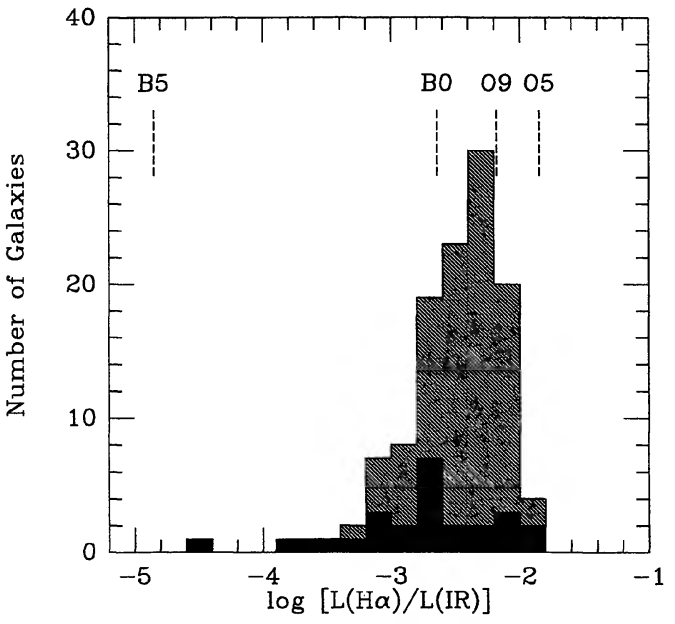

FIG. 9. Histogram of the ratio of $\mathrm{H} \alpha$ to far-infrared luminosity for all of the galaxies in this study (grey area), and for the galaxies with inclinations greater than $70^{\circ}$ (black area). Also included in the solid histogram are four peculiar galaxies which suffer severe extinction near the nuclei-NGC 520, 660,2146 , and Arp 220. The luminosity ratios expected for high mass stars are indicated by the dashed lines near the top.

Figures 10(a) and 10(b) show the ratio $L(\mathrm{H} \alpha) / L(\mathrm{IR})$ as a function of morphological type for the entire sample. It is clear that there is little difference among spiral galaxies of types Sab-Scd in the observed ratio of $\mathrm{H} \alpha$ to far-IR luminosity. The morphological categories which seem to be characterized by slightly elevated values of $L(\mathrm{H} \alpha) / L(\mathrm{IR})$ include types Sd-Im; conversely, the categories with slightly lower values of $L(\mathrm{H} \alpha) / L(\mathrm{IR})$ include types SO-Sa.

There are several reasons why one might observe a difference in the $L(\mathrm{H} \alpha) / L(\mathrm{IR})$ ratio between SO-Sa galaxies and Sd-Ir galaxies. Some of this tendency could be due to increased $\mathrm{H} \alpha$ absorption in the early-type galaxies by the underlying stellar population. Additional effects may be the result of metallicity variations along the Hubble sequence. As described by Devereux \& Young (1990a), the ratio $L(\mathrm{H} \alpha) / L(\mathrm{IR})$ is extremely sensitive to the mass of the highest mass stars which form, since the $\mathrm{H} \alpha$ to far-IR luminosity ratio spans more than a factor of 1000 for O5 through B5 stars. Therefore, it is reasonable to expect that the $L(\mathrm{H} \alpha)$ / $L(\mathrm{IR})$ ratio will also be sensitive to metallicity, since higher mass stars may form in regions of lower metallicity, according to the Jean's criterion. It is well known that Sd, Sdm, and Im galaxies are metal poor (cf. Pagel \& Edmunds 1981), so that stars of higher mass are likely to be forming in these systems. Another possibility is that the stars which form in the low metallicity systems have higher effective temperatures without an increase in the effective mass (cf. GarciaVargas 1995). Lastly, in regions of lower metallicity there is less dust present to absorb the bolometric luminosity, and this could lead to lower far-IR luminosities per unit $\mathrm{H} \alpha$ luminosity. Any of these possibilities could account for the slightly higher $L(\mathrm{H} \alpha) / L(\mathrm{IR})$ ratio observed in the Sd-Irr galaxies relative to the SO-Sa galaxies indicated in Fig. 10 .

Figure 10 also indicates that the $L(\mathrm{H} \alpha) / L(\mathrm{IR})$ ratio in the pairs and mergers is slightly lower than for the spirals. There are several possible explanations for this effect. First, the $\mathrm{H} \alpha$
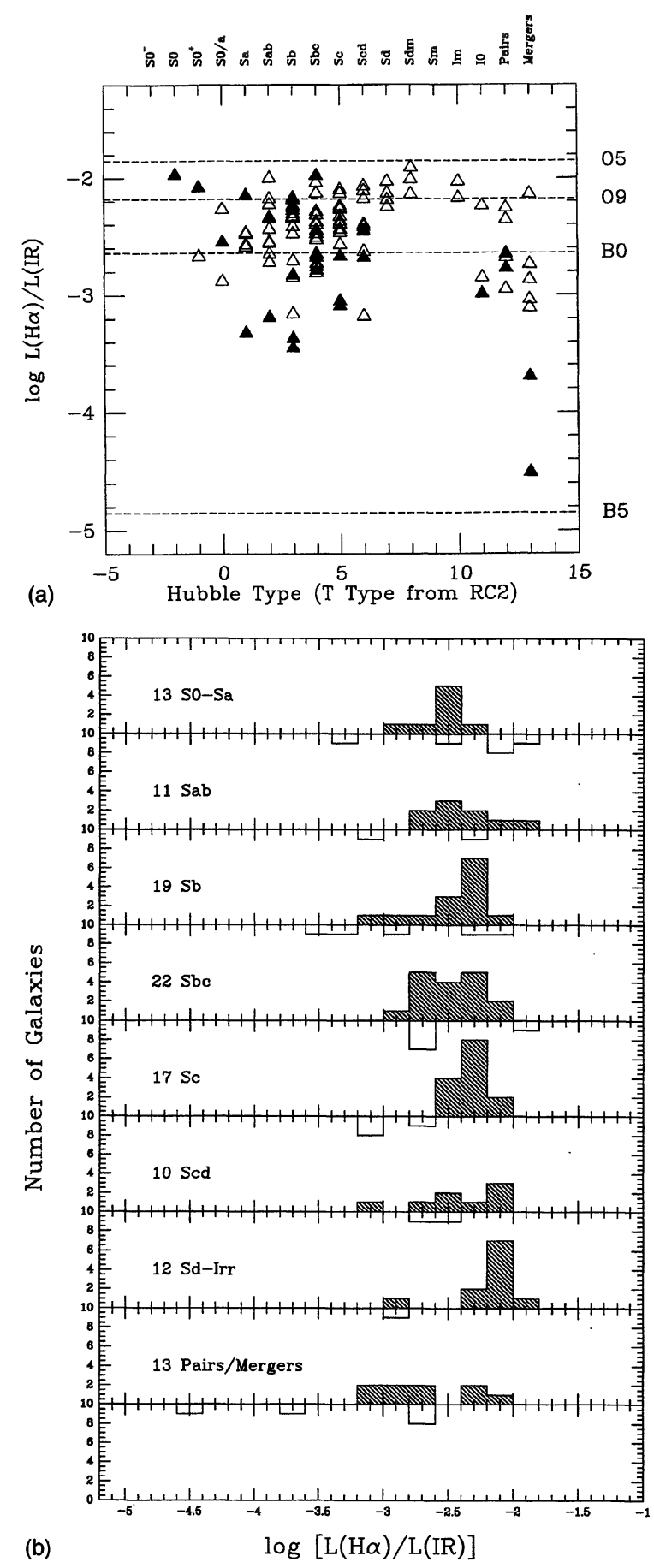

(b)

FIG. 10. (a) The ratio of $\mathrm{H} \alpha$ to far-infrared luminosity plotted as a function of morphological type. The galaxies with inclinations greater than $70^{\circ}$ are plotted as filled symbols. The ratios expected for high mass stars are indicated. Note that galaxies of types Sa through Scd exhibit similar ranges of observed values for the $\mathrm{H} \alpha$ to far-IR luminosity ratio. (b) Histograms of the ratio of $\mathrm{H} \alpha$ to far-infrared luminosity as a function of morphological type. The hatched histograms represent galaxies with inclinations $<70^{\circ}$, while the open histograms below represent the highly inclined and obscured galaxies. 
luminosity may be reduced because of extinction and geometry. Even though the highly inclined galaxies are indicated separately, there could be considerable extinction even in systems with smaller inclinations, partly because these galaxies have unusual morphologies and it is difficult to determine a meaningful inclination, and partly because there may be unusually high gas concentrations (and extinction) in the inner regions of these galaxies. Another trend which could be contributing to the lower $L(\mathrm{H} \alpha) / L(\mathrm{IR})$ ratios in the pairs and mergers is that these galaxies may have enhanced IR luminosities due to the presence of a nonthermal source in the nucleus.

\section{THE GLOBAL STAR FORMATION EFFICIENCY IN GALAXIES}

\subsection{Definition of the Star Formation Efficiency}

The star formation efficiency (SFE) is a measure of the yield of young high mass stars per unit mass of gas available to form those stars. In the following analysis, we investigate the global star formation efficiency measured in two different ways-first, using the $\mathrm{H} \alpha$ line luminosity to trace the yield of young stars, and second, using the far-infrared luminosity-both normalized by the global mass of molecular gas. We examine the trends in the global star formation efficiency as a function of morphology along the Hubble sequence and as a function of galaxy environment for both of the above measures of the SFE. One consequence of the comparisons we show is the illustration that the FIR luminosity is an equally good tracer of the high mass star formation rate as the $\mathrm{H} \alpha$ luminosity.

Table 4 presents the global star formation efficiencies for the galaxies in this study sorted by morphological type. The information in this table includes the galaxy type, inclination, and environment, as well as the global ratios $L(\mathrm{H} \alpha) / \mathscr{M}\left(\mathrm{H}_{2}\right), L(\mathrm{IR}) / \mathscr{M}\left(\mathrm{H}_{2}\right)$, and $S(60) / S(100)$. We remind the reader that the global molecular gas masses were derived from major axis $\mathrm{CO}$ observations (see Sec. 3.2), while the global $\mathrm{H} \alpha$ and IR luminosities are derived by integrating the flux of the entire galaxy. In a later paper, we will present the comparison of $\mathrm{H} \alpha$ and $\mathrm{CO}$ fluxes in the same $45^{\prime \prime}$ apertures within and among these galaxies.

Figure 11 shows a comparison of two different measures of the global star formation efficiency. This plot of $L(\mathrm{H} \alpha) / \mathscr{M}\left(\mathrm{H}_{2}\right)$ vs $L(\mathrm{IR}) / \mathscr{C}\left(\mathrm{H}_{2}\right)$ is presented with the galaxies coded by morphological type. There is a clear correlation between these two quantities for all morphologies, in that galaxies with elevated values of $L(\mathrm{H} \alpha) / \mathscr{C}\left(\mathrm{H}_{2}\right)$ also have elevated values of $L(\mathrm{H} \alpha) / \mathscr{C}\left(\mathrm{H}_{2}\right)$. Since the $\mathrm{H} \alpha$ luminosity in spiral galaxies is from $\mathrm{H} \mathrm{II}$ regions and associated with high mass star formation, the correlation of $L(\mathrm{H} \alpha) / \mathscr{M}\left(\mathrm{H}_{2}\right)$ with $L(\mathrm{IR}) / \mathscr{M}\left(\mathrm{H}_{2}\right)$ strongly supports the suggestion that the IR luminosity also traces high mass star formation.

\subsection{Dependence of the SFE on Morphological Type}

Figure 12 shows histograms of the global $L(\mathrm{H} \alpha) / \mathscr{M}\left(\mathrm{H}_{2}\right)$ ratio as a function of morphological type for the 96 galaxies in our sample with inclinations less than $70^{\circ}$. This figure illustrates that, for the galaxies in our sample, the mean rate of high mass star formation per unit mass of molecular gas shows little variation with morphological type for types $\mathrm{Sa}$ through Sc. For comparison, we also show the distributions of $L_{\mathrm{IR}} / \mathscr{U}\left(\mathrm{H}_{2}\right)$ for the same galaxies as a function of morphological type. These distributions also indicate a similar mean value of the star formation rate per unit mass of molecular gas from type to type for S0 through Scd galaxies. We conclude that Hubble type plays little role, if any, in determining the mean yield of high mass stars per unit mass of molecular gas in these galaxies.

Table 5 summarizes the mean values of the SFE for the galaxies in our sample.

Among the latest type spiral galaxies (Sd-Sm) and among the irregular galaxies, the mean ratios of both $L(\mathrm{H} \alpha) / \mathscr{M}\left(\mathrm{H}_{2}\right)$ and $L_{\mathrm{IR}} / \mathscr{M}\left(\mathrm{H}_{2}\right)$ are higher than for any of the other morphological categories. These galaxies tend to be characterized by low luminosities, small sizes, and low metallicities. It is possible that the molecular gas masses for these galaxies have been underestimated, leading to an overestimate of the mean ratios of both $L(\mathrm{H} \alpha) / \mathscr{M}\left(\mathrm{H}_{2}\right)$ and $L_{\mathrm{IR}} / \mathscr{U}\left(\mathrm{H}_{2}\right)$. However, independent of the $\mathrm{H}_{2}$ mass estimates, we note that the ratio of $L(\mathrm{H} \alpha) / L(\mathrm{IR})$ is elevated in the irregular galaxies [cf. Sec. 4.3 above and Figs. 10(a) and 10(b)]. Thus, the results of the comparison of the $\mathrm{H} \alpha$ luminosities, IR luminosities, and molecular gas masses indicates that the late type spirals (Sd-Sm) and the irregulars differ, in fact, when compared with the majority of the spiral galaxies.

One notable difference between the $L(\mathrm{H} \alpha) / \mathscr{Q} 6\left(\mathrm{H}_{2}\right)$ and the $L_{\mathrm{IR}} / \mathscr{C}\left(\mathrm{H}_{2}\right)$ distributions is that more extreme values in the ratio are found for $L(\mathrm{H} \alpha) / \mathscr{C}\left(\mathrm{H}_{2}\right)$ within each morphological type than for $L_{\mathrm{IR}} / \mathscr{C}\left(\mathrm{H}_{2}\right)$. Extinction within these galaxies will certainly introduce some additional dispersion in $L(\mathrm{H} \alpha) / \mathscr{C}\left(\mathrm{H}_{2}\right)$ relative to $L_{\mathrm{IR}} / \mathscr{U}\left(\mathrm{H}_{2}\right)$, especially since the more inclined galaxies in the sample (i.e., the $\mathrm{Sb}$ galaxy NGC 1055 with $i=69^{\circ}$ ) can suffer severe extinction. Thus, some of the observed scatter in $L(\mathrm{H} \alpha) / \mathscr{O}\left(\mathrm{H}_{2}\right)$ is a result of extinction, some of the scatter is a result of measurement uncertainties, and some reflects real differences in the star formation efficiency from galaxy to galaxy.

\subsection{Effects of Environment}

Galaxy environment has long been known to play an important role in the evolution of galaxies (Arp 1966; Toomre \& Toomre 1972; Larson \& Tinsley 1978). Recent studies of the effects of galaxy-galaxy interactions on the rate of star formation (Bushouse 1987; Kennicutt et al. 1987) and its efficiency in galaxies (Young et al. 1986a, 1968b; Sanders et al. 1986; Solomon \& Sage 1988; Tinney et al. 1990) show that both the rates and efficiencies of high mass star formation are elevated in interacting systems.

The sample of galaxies which we have imaged in $\mathrm{H} \alpha$ includes galaxies in a wide range of environments: mergers, close pairs, Virgo spirals, group members, field spirals, and isolated galaxies. Nine systems are classified as mergers, based on the presence of tidal tails or other morphological peculiarities (Schweizer et al. 1983; Joseph \& Wright 1985; Sanders et al. 1987), and an additional six close pairs are included (pairs in which the angular separation between members is $<3^{\prime}$, so both galaxies appear in the same CCD field). The ten isolated spiral galaxies in our study were cho- 
TABLE 4. Global galaxy properties as a function of morphological type.

\begin{tabular}{|c|c|c|c|c|c|c|c|}
\hline $\begin{array}{l}\text { Morphology } \\
\text { (1) }\end{array}$ & $\begin{array}{c}\text { Galaxy } \\
\text { (2) }\end{array}$ & $\begin{array}{l}\text { Type } \\
\text { (3) }\end{array}$ & $\begin{array}{l}\text { Inc. } \\
(9) \\
(4)\end{array}$ & (5) & $\begin{array}{c}\log \\
L(H \alpha) / M\left(H_{2}\right) \\
\left(L_{\alpha^{\prime}} / M_{0}\right) \\
(6)\end{array}$ & $\begin{array}{c}\log \\
L(I R) / M\left(H_{2}\right) \\
\left(L_{\sigma^{\prime}} / M_{0}\right) \\
(7)\end{array}$ & $\begin{array}{c}S(60) \\
S(100) \\
(8)\end{array}$ \\
\hline SO \& SO/a & $\begin{array}{l}\text { NGC } 3593 \\
\text { NGC } 4293 \\
\text { NGC } 4438 \\
\text { NGC } 4459 \\
\text { NGC } 4526 \\
\text { NGC } 4710\end{array}$ & $\begin{array}{c}\text { SA(s)0/a } \\
\text { (R)SB(s)0/a } \\
\text { SA(s)0/a pec } \\
\text { SA(s)0+ } \\
\text { SAB(S)0 } \\
\text { SA(r)0+ sp }\end{array}$ & $\begin{array}{l}67 \\
76 \\
68 \\
43 \\
75 \\
79\end{array}$ & $\begin{array}{l}\text { Field } \\
\text { Virgo } \\
\text { Virgo } \\
\text { Virgo } \\
\text { Virgo } \\
\text { Virgo }\end{array}$ & $\begin{array}{r}-2.23 \\
-1.90 \\
-1.50 \\
>-2.14 \\
>-0.73 \\
-1.24\end{array}$ & $\begin{array}{r}0.65 \\
0.65 \\
0.77 \\
>0.53 \\
>>1.24 \\
0.84\end{array}$ & $\begin{array}{l}0.52 \\
0.45 \\
0.42 \\
0.36 \\
0.38 \\
0.47\end{array}$ \\
\hline $\mathbf{S a}$ & $\begin{array}{l}\text { NGC } 3623 \\
\text { NGC } 3718 \\
\text { NGC } 4064 \\
\text { NGC } 4419 \\
\text { NGC } 4424 \\
\text { NGC } 7625\end{array}$ & $\begin{array}{c}\text { SAB(rs)a } \\
\text { SB(s)a pec } \\
\text { SB(s)a pec } \\
\text { SB(s)a sp } \\
\text { SB(s)a } \\
\text { SA(rs)a pec }\end{array}$ & $\begin{array}{l}71 \\
61 \\
67 \\
67 \\
61 \\
22\end{array}$ & $\begin{array}{l}\text { Group } \\
\text { Field } \\
\text { Virgo } \\
\text { Virgo } \\
\text { Virgo } \\
\text { Field }\end{array}$ & $\begin{array}{r}>-1.92 \\
>-2.40 \\
-1.62 \\
-2.32 \\
-1.39 \\
-1.72\end{array}$ & $\begin{array}{r}>0.23 \\
>0.08 \\
0.95 \\
0.27 \\
1.08 \\
0.85\end{array}$ & $\begin{array}{l}0.23 \\
0.36 \\
0.48 \\
0.50 \\
0.55 \\
0.51\end{array}$ \\
\hline Sab & $\begin{array}{c}\text { NGC } 2775 \\
\text { NGC } 3504 \\
\text { NGC } 4192 \\
\text { NGC } 4351 \\
\text { NGC } 4450 \\
\text { NGC } 4569 \\
\text { NGC } 4736 \\
\text { NGC } 4826 \\
\text { NGC } 7217 \\
\text { IC } 356\end{array}$ & $\begin{array}{c}\text { SA(r)ab } \\
\text { (R)SAB(s)ab } \\
\text { SAB(s)ab } \\
\text { SB(rs)ab pec } \\
\text { SA(s)ab } \\
\text { SAB(rs)ab } \\
\text { (R)SA(r)ab } \\
\text { (R)SA(rs)ab } \\
\text { (R)SA(r)ab } \\
\text { SA(s)ab pec }\end{array}$ & $\begin{array}{l}45 \\
36 \\
74 \\
46 \\
45 \\
63 \\
35 \\
57 \\
32 \\
40\end{array}$ & $\begin{array}{c}\text { Isolated } \\
\text { Field } \\
\text { Virgo } \\
\text { Virgo } \\
\text { Virgo } \\
\text { Virgo } \\
\text { Field } \\
\text { Field } \\
\text { Isolated } \\
\text { Field }\end{array}$ & \begin{tabular}{r|}
-1.89 \\
-1.56 \\
-1.95 \\
-1.81 \\
-2.38 \\
-1.96 \\
-1.22 \\
-1.96 \\
-1.78 \\
-2.35
\end{tabular} & $\begin{array}{r}0.29 \\
1.01 \\
0.39 \\
>0.54 \\
0.27 \\
0.27 \\
0.77 \\
0.59 \\
0.66 \\
0.37\end{array}$ & $\begin{array}{l}0.23 \\
0.65 \\
0.37 \\
0.33 \\
0.21 \\
0.40 \\
0.54 \\
0.52 \\
0.29 \\
0.24\end{array}$ \\
\hline $\mathbf{S b}$ & $\begin{array}{l}\text { NGC } 278 \\
\text { NGC } 488 \\
\text { NGC } 891 \\
\text { NGC } 1055 \\
\text { NGC } 1068 \\
\text { NGC } 2683 \\
\text { NGC } 2841 \\
\text { NGC } 3351 \\
\text { NGC } 3627 \\
\text { NGC } 3675 \\
\text { NGC } 4102 \\
\text { NGC } 4157 \\
\text { NGC } 4216 \\
\text { NGC } 4380 \\
\text { NGC } 4394 \\
\text { NGC } 4413 \\
\text { NGC } 4501 \\
\text { NGC } 4548 \\
\text { NGC } 4579\end{array}$ & $\begin{array}{c}\text { SAB(rs)b } \\
\text { SA(r)b } \\
\text { SA(s)b sp } \\
\text { SBb sp } \\
\text { (R)SA(rs)b } \\
\text { SA(rs)b } \\
\text { SA(r)b } \\
\text { SB(r)b } \\
\text { SAB(s)b } \\
\text { SA(s)b } \\
\text { SAB(s)b } \\
\text { SAB(s)b sp } \\
\text { SAB(s)b } \\
\text { SA(rs)b } \\
\text { (R)SB(r)b } \\
\text { (R)SB(rs)b } \\
\text { SA(rs)b } \\
\text { SB(rs)b } \\
\text { SAB(rs)b }\end{array}$ & $\begin{array}{l}12 \\
40 \\
86 \\
69 \\
40 \\
80 \\
64 \\
48 \\
60 \\
59 \\
54 \\
82 \\
80 \\
55 \\
25 \\
49 \\
58 \\
37 \\
37\end{array}$ & $\begin{array}{l}\text { Field } \\
\text { Field } \\
\text { Field } \\
\text { Field } \\
\text { Field } \\
\text { Field } \\
\text { Field } \\
\text { Field } \\
\text { Group } \\
\text { Field } \\
\text { Field } \\
\text { Field } \\
\text { Virgo } \\
\text { Virgo } \\
\text { Virgo } \\
\text { Virgo } \\
\text { Virgo } \\
\text { Virgo } \\
\text { Virgo }\end{array}$ & $\begin{array}{c}-1.31 \\
-2.56 \\
-2.81 \\
-3.01 \\
-1.59 * \\
-1.31 \\
-2.22 \\
-1.47 \\
-1.96 \\
-1.91 \\
-2.03 \\
-2.27 \\
-1.86 \\
>-1.90 \\
-1.96 \\
>-1.18 \\
-2.03 \\
-2.35 \\
-1.99\end{array}$ & $\begin{array}{r}1.03 \\
0.29 \\
0.64 \\
0.35 \\
0.83 \\
0.97 \\
0.11 \\
0.78 \\
0.52 \\
0.40 \\
1.13 \\
0.56 \\
0.31 \\
>0.41 \\
0.23 \\
>1.06 \\
0.45 \\
0.36 \\
0.36\end{array}$ & $\begin{array}{l}0.57 \\
0.21 \\
0.36 \\
0.37 \\
0.79 \\
0.29 \\
0.21 \\
0.51 \\
0.46 \\
0.29 \\
0.68 \\
0.35 \\
0.21 \\
0.29 \\
0.21 \\
0.35 \\
0.32 \\
0.21 \\
0.29\end{array}$ \\
\hline Sbc & $\begin{array}{l}\text { NGC } 157 \\
\text { NGC } 2336 \\
\text { NGC } 2339 \\
\text { NGC } 2903 \\
\text { NGC } 3147 \\
\text { NGC } 3521 \\
\text { NGC } 4041 \\
\text { NGC } 4088 \\
\text { NGC } 4206 \\
\text { NGC } 4212 \\
\text { NGC } 4237 \\
\text { NGC } 4303 \\
\text { NGC } 4321 \\
\text { NGC } 4527 \\
\text { NGC } 4536 \\
\text { NGC } 4639\end{array}$ & $\begin{array}{c}\text { SAB(rs)bc } \\
\text { SAB(r)bc } \\
\text { SAB(rs)bc } \\
\text { SAB(rs)bc } \\
\text { SA(rs)bc } \\
\text { SAB(rs)bc } \\
\text { SA(rs)bc } \\
\text { SAB(rs)bc } \\
\text { SA(s)bc } \\
\text { SAbc } \\
\text { SAB(rs)bc } \\
\text { SAB(rs)bc } \\
\text { SAB(s)bc } \\
\text { SAB(s)bc } \\
\text { SAB(rs)bc } \\
\text { SAB(rs)bc }\end{array}$ & $\begin{array}{l}49 \\
56 \\
40 \\
60 \\
28 \\
60 \\
18 \\
67 \\
83 \\
47 \\
47 \\
25 \\
28 \\
72 \\
67 \\
45\end{array}$ & $\begin{array}{c}\text { Isolated } \\
\text { Field } \\
\text { Field } \\
\text { Isolated } \\
\text { Isolated } \\
\text { Isolated } \\
\text { Field } \\
\text { Field } \\
\text { Virgo } \\
\text { Virgo } \\
\text { Virgo } \\
\text { Virgo } \\
\text { Virgo } \\
\text { Virgo } \\
\text { Virgo } \\
\text { Virgo }\end{array}$ & \begin{tabular}{r|}
-1.35 \\
-1.39 \\
-1.98 \\
-1.64 \\
-2.47 \\
-2.04 \\
-1.65 \\
-1.77 \\
$>-1.63$ \\
-1.98 \\
-1.59 \\
-1.56 \\
-2.16 \\
-2.08 \\
-1.75 \\
$>-1.18$
\end{tabular} & $\begin{array}{r}0.93 \\
0.23 \\
0.68 \\
0.74 \\
0.34 \\
0.41 \\
0.67 \\
0.74 \\
>0.35 \\
0.55 \\
1.09 \\
0.57 \\
0.32 \\
0.60 \\
0.90 \\
>0.86\end{array}$ & $\begin{array}{l}0.42 \\
0.27 \\
0.58 \\
0.42 \\
0.28 \\
0.42 \\
0.45 \\
0.45 \\
0.45 \\
0.41 \\
0.30 \\
0.48 \\
0.37 \\
0.51 \\
0.69 \\
0.36\end{array}$ \\
\hline
\end{tabular}


TABLE 4. (continued)

\begin{tabular}{|c|c|c|c|c|c|c|c|}
\hline $\begin{array}{c}\text { Morphology } \\
\text { (1) }\end{array}$ & $\begin{array}{c}\text { Galaxy } \\
\text { (2) }\end{array}$ & $\begin{array}{l}\text { Type } \\
\text { (3) }\end{array}$ & $\begin{array}{l}\text { Inc. } \\
\left({ }^{\circ}\right) \\
(4)\end{array}$ & $\begin{array}{l}\text { Env. } \\
\text { (5) }\end{array}$ & $\begin{array}{c}\log \\
L(H \alpha) / M\left(H_{2}\right) \\
\left(L_{\sigma_{0}} M_{0}\right) \\
(6)\end{array}$ & $\begin{array}{c}\log \\
\mathrm{L}(\mathrm{IR}) / \mathrm{M}\left(\mathrm{H}_{2}\right) \\
\left(\mathrm{L}_{\sigma} \mathrm{M}_{\mathrm{O}}\right) \\
(7)\end{array}$ & $\begin{array}{c}S(60) \\
S(100) \\
(8)\end{array}$ \\
\hline $\begin{array}{l}\text { Sb } \\
\text { continued }\end{array}$ & $\begin{array}{l}\text { NGC } 4689 \\
\text { NGC } 5005 \\
\text { NGC } 5248 \\
\text { NGC } 6951 \\
\text { NGC } 7331 \\
\text { NGC } 7541\end{array}$ & $\begin{array}{c}\text { SA(rs)bc } \\
\text { SAB(rs)bc } \\
\text { SAB(rs)bc } \\
\text { SAB(rs)bc } \\
\text { SA(s)bc } \\
\text { SB(rs)bc pec }\end{array}$ & $\begin{array}{l}30 \\
62 \\
42 \\
30 \\
71 \\
70\end{array}$ & $\begin{array}{l}\text { Virgo } \\
\text { Field } \\
\text { Field } \\
\text { Isolated } \\
\text { Field } \\
\text { Field }\end{array}$ & $\begin{array}{l}-2.24 \\
-2.02 \\
-1.77 \\
-2.28 \\
-2.21 \\
-1.95\end{array}$ & $\begin{array}{l}0.14 \\
0.71 \\
0.63 \\
0.48 \\
0.44 \\
0.84\end{array}$ & $\begin{array}{l}0.30 \\
0.35 \\
0.43 \\
0.38 \\
0.36 \\
0.48\end{array}$ \\
\hline Se & $\begin{array}{l}\text { NGC } 253 \\
\text { NGC } 628 \\
\text { NGC } 1084 \\
\text { NGC } 1087 \\
\text { NGC } 1961 \\
\text { NGC } 2276 \\
\text { NGC } 2976 \\
\text { NGC } 3079 \\
\text { NGC } 3631 \\
\text { NGC } 3810 \\
\text { NGC } 4254 \\
\text { NGC } 4535 \\
\text { NGC } 4647 \\
\text { NGC } 4651 \\
\text { NGC } 5907 \\
\text { NGC } 6643 \\
\text { NGC } 7479\end{array}$ & $\begin{array}{l}\text { SAB(s)c } \\
\text { SA(s)c } \\
\text { SA(s)c } \\
\text { SAB(rs)c } \\
\text { SAB(rs)c } \\
\text { SAB(rs)c } \\
\text { SAc pec } \\
\text { SB(s)c sp } \\
\text { SA(s)c } \\
\text { SA(rs)c } \\
\text { SA(s)c } \\
\text { SAB(s)c } \\
\text { SAB(rs)c } \\
\text { SA(rs)c } \\
\text { SA(s)c sp } \\
\text { SA(rs)c } \\
\text { SB(s)c }\end{array}$ & $\begin{array}{l}78 \\
22 \\
59 \\
49 \\
46 \\
18 \\
61 \\
77 \\
28 \\
45 \\
28 \\
43 \\
36 \\
46 \\
89 \\
59 \\
38\end{array}$ & $\begin{array}{l}\text { Field } \\
\text { Field } \\
\text { Field } \\
\text { Field } \\
\text { Field } \\
\text { Field } \\
\text { Field } \\
\text { Field } \\
\text { Field } \\
\text { Field } \\
\text { Virgo } \\
\text { Virgo } \\
\text { Virgo } \\
\text { Virgo } \\
\text { Field } \\
\text { Field } \\
\text { Isolated }\end{array}$ & $\begin{array}{l}-2.06 \\
-1.78 \\
-1.64 \\
-1.50 \\
-1.92 \\
-1.67 \\
-1.36 \\
-2.36 \\
-1.80 \\
-1.71 \\
-1.91 \\
-2.00 \\
-1.99 \\
-1.71 \\
-2.11 \\
-1.55 \\
-2.12\end{array}$ & $\begin{array}{l}1.03 \\
0.49 \\
0.83 \\
0.87 \\
0.46 \\
0.59 \\
0.75 \\
0.68 \\
0.33 \\
0.53 \\
0.48 \\
0.33 \\
0.44 \\
0.68 \\
0.57 \\
0.89 \\
0.45\end{array}$ & $\begin{array}{l}0.63 \\
0.37 \\
0.50 \\
0.43 \\
0.30 \\
0.45 \\
0.38 \\
0.49 \\
0.35 \\
0.40 \\
0.40 \\
0.37 \\
0.35 \\
0.37 \\
0.23 \\
0.35 \\
0.56\end{array}$ \\
\hline Sed & $\begin{array}{c}\text { NGC } 3184 \\
\text { NGC } 3556 \\
\text { NGC } 4189 \\
\text { NGC } 4294 \\
\text { NGC } 4498 \\
\text { NGC } 4522 \\
\text { NGC } 4654 \\
\text { NGC } 4808 \\
\text { NGC } 6946 \\
\text { IC } 342\end{array}$ & $\begin{array}{l}\text { SAB(rs)cd } \\
\text { SB(s)cd sp } \\
\text { SAB(rs)cd } \\
\text { SB(s)cd } \\
\text { SAB(s)cd } \\
\text { SB(s)cd sp } \\
\text { SAB(rs)cd } \\
\text { SA(s)cd } \\
\text { SAB(rs)cd } \\
\text { SAB(rs)cd }\end{array}$ & $\begin{array}{l}12 \\
77 \\
30 \\
67 \\
56 \\
76 \\
52 \\
67 \\
30 \\
25\end{array}$ & $\begin{array}{c}\text { Field } \\
\text { Isolated } \\
\text { Virgo } \\
\text { Virgo } \\
\text { Virgo } \\
\text { Virgo } \\
\text { Virgo } \\
\text { Virgo } \\
\text { Isolated } \\
\text { Field }\end{array}$ & $\begin{array}{r}-1.71 \\
-1.68 \\
-1.84 \\
>-0.80 \\
>-1.64 \\
>-1.95 \\
-1.91 \\
>-1.17 \\
-1.98 \\
-2.80\end{array}$ & $\begin{array}{r}0.41 \\
1.00 \\
0.59 \\
>1.27 \\
>0.54 \\
>0.51 \\
0.72 \\
>1.23 \\
0.47 \\
0.38\end{array}$ & $\begin{array}{l}0.31 \\
0.47 \\
0.38 \\
0.51 \\
0.31 \\
0.36 \\
0.37 \\
0.43 \\
0.47 \\
0.46\end{array}$ \\
\hline Sd & $\begin{array}{l}\text { NGC } 925 \\
\text { NGC } 4519 \\
\text { NGC } 4571 \\
\text { NGC } 4713\end{array}$ & $\begin{array}{l}\text { SAB(s)d } \\
\text { SB(rs)d } \\
\text { SA(r)d } \\
\text { SAB(rs)d }\end{array}$ & $\begin{array}{l}53 \\
45 \\
38 \\
49\end{array}$ & $\begin{array}{l}\text { Field } \\
\text { Virgo } \\
\text { Virgo } \\
\text { Virgo }\end{array}$ & $\begin{array}{r}>-1.59 \\
>-1.38 \\
-2.03 \\
>-0.91\end{array}$ & $\begin{array}{r}>0.44 \\
>0.82 \\
0.22 \\
>1.22\end{array}$ & $\begin{array}{l}0.34 \\
0.56 \\
0.24 \\
0.46\end{array}$ \\
\hline Sdm \& Sm & $\begin{array}{l}\text { NGC } 4178 \\
\text { NGC } 4299 \\
\text { NGC } 4561\end{array}$ & $\begin{array}{l}\text { SB(rs)dm } \\
\text { SAB(s)dm } \\
\text { SB(rs)dm }\end{array}$ & $\begin{array}{l}69 \\
22 \\
34\end{array}$ & $\begin{array}{l}\text { Virgo } \\
\text { Virgo } \\
\text { Virgo }\end{array}$ & $\begin{array}{r}-0.91 \\
>-0.71 \\
>-1.60\end{array}$ & $\begin{array}{r}1.22 \\
>1.20 \\
>0.41\end{array}$ & $\begin{array}{l}0.43 \\
0.55 \\
0.43\end{array}$ \\
\hline 10 & $\begin{array}{r}\text { NGC } 972 \\
\text { NGC } 3034 \\
\text { NGC } 3077\end{array}$ & $\begin{array}{c}10 \\
\text { I0 sp } \\
10 \text { pec }\end{array}$ & $\begin{array}{l}59 \\
80 \\
38\end{array}$ & $\begin{array}{l}\text { Field } \\
\text { Group } \\
\text { Group }\end{array}$ & $\begin{array}{l}-1.86 \\
-1.83 * \\
-0.76\end{array}$ & $\begin{array}{l}0.99 \\
1.16 \\
1.48\end{array}$ & $\begin{array}{l}0.55 \\
1.11 \\
0.58\end{array}$ \\
\hline Im & $\begin{array}{l}\text { NGC } 1569 \\
\text { NGC } 4532\end{array}$ & $\begin{array}{l}\mathrm{IBm} \\
\mathrm{IBm}\end{array}$ & $\begin{array}{l}61 \\
67\end{array}$ & $\begin{array}{l}\text { Field } \\
\text { Virgo }\end{array}$ & $\begin{array}{r}+0.15 \\
>-0.67\end{array}$ & $\begin{array}{r}2.18 \\
>1.49\end{array}$ & $\begin{array}{l}1.07 \\
0.60\end{array}$ \\
\hline $\begin{array}{l}\text { Isolated } \\
\text { Galaxies }\end{array}$ & $\begin{array}{l}\text { NGC } 157 \\
\text { NGC } 2775 \\
\text { NGC } 2903 \\
\text { NGC } 3147 \\
\text { NGC } 3521 \\
\text { NGC } 3556 \\
\text { NGC } 6946 \\
\text { NGC } 6951\end{array}$ & $\begin{array}{l}\text { SAB(rs)bc } \\
\text { SA(r)ab } \\
\text { SAB(rs)bc } \\
\text { SA(rs)bc } \\
\text { SAB(rs)bc } \\
\text { SB(s)cd sp } \\
\text { SAB(rs)cd } \\
\text { SAB(rs)bc }\end{array}$ & $\begin{array}{l}49 \\
45 \\
60 \\
28 \\
60 \\
77 \\
30 \\
30\end{array}$ & $\begin{array}{l}\text { Isolated } \\
\text { Isolated } \\
\text { Isolated } \\
\text { Isolated } \\
\text { Isolated } \\
\text { Isolated } \\
\text { Isolated } \\
\text { Isolated }\end{array}$ & $\begin{array}{l}-1.35 \\
-1.89 \\
-1.64 \\
-2.47 \\
-2.04 \\
-1.68 \\
-1.98 \\
-2.28\end{array}$ & $\begin{array}{l}0.93 \\
0.29 \\
0.74 \\
0.34 \\
0.41 \\
1.00 \\
0.47 \\
0.48\end{array}$ & $\begin{array}{l}0.42 \\
0.23 \\
0.42 \\
0.28 \\
0.42 \\
0.47 \\
0.47 \\
0.38\end{array}$ \\
\hline
\end{tabular}


TABLE 4. (continued)

\begin{tabular}{|c|c|c|c|c|c|c|c|}
\hline $\begin{array}{l}\text { Category } \\
\text { (1) }\end{array}$ & $\begin{array}{c}\text { Galaxy } \\
\text { (2) }\end{array}$ & $\begin{array}{l}\text { Type } \\
\text { (3) }\end{array}$ & $\begin{array}{l}\text { Inc. } \\
\left({ }^{\circ}\right) \\
(4)\end{array}$ & $\begin{array}{l}\text { Env. } \\
\text { (5) }\end{array}$ & $\begin{array}{c}\log \\
\mathrm{L}(\mathrm{H} \alpha) / \mathrm{M}\left(\mathrm{H}_{2}\right) \\
\left(\mathrm{L}_{\mathbf{o}^{\prime}} \mathrm{M}_{\mathrm{O}}\right) \\
(6)\end{array}$ & $\begin{array}{c}\log \\
L(\mathbb{R}) / M_{\left(H_{2}\right)} \\
\left(L_{\alpha_{0}} M_{0}\right) \\
(7)\end{array}$ & $\begin{array}{c}S(60) \\
S(100) \\
(8)\end{array}$ \\
\hline $\begin{array}{l}\text { Isolated } \\
\text { Galaxies }\end{array}$ & $\begin{array}{l}\text { NGC } 7217 \\
\text { NGC } 7479\end{array}$ & $\begin{array}{c}\text { (R)SA(r)ab } \\
\text { SB(s)c }\end{array}$ & $\begin{array}{l}32 \\
38\end{array}$ & $\begin{array}{l}\text { Isolated } \\
\text { Isolated }\end{array}$ & $\begin{array}{l}-1.78 \\
-2.12\end{array}$ & $\begin{array}{l}0.66 \\
0.45\end{array}$ & $\begin{array}{l}0.29 \\
0.56\end{array}$ \\
\hline Pairs & $\begin{array}{c}\text { NGC } 2798 / 99 \\
\text { NGC } 3690 / 1 C 694 \\
\text { NGC } 4038 / 39 \\
\text { NGC } 4298 / 4302 \\
\text { NGC } 4567 / 4568 \\
\text { NGC } 5194\end{array}$ & $\begin{array}{c}\text { SB(s)a pec } \\
\text { IBm pec } \\
\text { SB(s)m pec } \\
\text { SA(rs)c } \\
\text { SA(rs)bc } \\
\text { SA(s)bc pec }\end{array}$ & $\begin{array}{l}68 \\
34 \\
50 \\
67 \\
46 \\
20\end{array}$ & $\begin{array}{l}\text { Pair } \\
\text { Pair } \\
\text { Pair } \\
\text { Pair } \\
\text { Pair } \\
\text { Pair }\end{array}$ & $\begin{array}{l}-1.90 \\
-1.58 \\
-1.64 \\
-2.39 \\
-2.11 \\
-1.85\end{array}$ & $\begin{array}{l}0.87 \\
1.37 \\
0.63 \\
0.26 \\
0.56 \\
0.51\end{array}$ & $\begin{array}{l}0.71 \\
1.00 \\
0.52 \\
0.24 \\
0.37 \\
0.44\end{array}$ \\
\hline Mergers & $\begin{array}{c}\text { NGC } 520 \\
\text { NGC } 660 \\
\text { NGC } 1614 \\
\text { NGC } 2146 \\
\text { NGC } 3310 \\
\text { NGC } 4194 \\
\text { NGC } 6240 \\
\text { Arp } 220 \\
\text { Mrk } 231\end{array}$ & $\begin{array}{c}\text { Pec } \\
\text { SB(s)a pec } \\
\text { SB(s)c pec } \\
\text { SB(s)ab pec } \\
\text { SAB(r)bc pec } \\
\text { IBm pec } \\
\text { I0 pec } \\
\text { Pec } \\
\text { SA(rs)c pec }\end{array}$ & $\begin{array}{l}66 \\
66 \\
30 \\
52 \\
36 \\
49 \\
60 \\
\ddot{49}\end{array}$ & $\begin{array}{c}\text { Merger } \\
\text { Merger? } \\
\text { Merger } \\
\text { Merger? } \\
\text { Merger } \\
\text { Merger } \\
\text { Merger } \\
\text { Merger } \\
\text { Merger? }\end{array}$ & $\begin{array}{l}-3.04 * \\
-2.63 * \\
-1.83 \\
-2.22 * \\
-0.51 \\
-1.48 \\
-1.62 \\
-2.99 * \\
-1.28\end{array}$ & $\begin{array}{l}0.66 \\
0.70 \\
1.28 \\
0.96 \\
1.63 \\
1.39 \\
1.12 \\
1.52 \\
1.76\end{array}$ & $\begin{array}{l}0.67 \\
0.76 \\
0.95 \\
0.74 \\
0.76 \\
0.99 \\
0.83 \\
0.91 \\
1.08\end{array}$ \\
\hline
\end{tabular}

Col. (1) Overall morphological category.

Notes to Table 4

Col. (2) Galaxy name-NGC, IC, Mrk, or Arp designation.

Col. (3) Morphological type from Table 1.

Col. (4) Galaxy inclination in degrees, derived from the ratio of major to minor axes listed in the RC2 [ $\log R(25)]$ or UGC, and computed following Roberts (1969). The inclination is given by: $\cos ^{2} i=1.042\left\{10^{\log R(25)}\right\}^{-2}-0.042$. This formulation accounts for the finite thickness of a galaxy even when oriented edge-on.

Col. (5) Galaxy environment. Galaxies listed as Isolated have no neighbors within $500 \mathrm{~km} / \mathrm{sec}$ and $1 \mathrm{Mpc}$ projected on the sky. Galaxies listed as Group members are found in the M81 Group or the Leo Triplet. Galaxies listed as Virgo are members of the Virgo Cluster (cf. Kenney and Young 1988). Galaxies listed as Field are not in any of the other categories. Galaxies listed as Pairs refer to two galaxies in contact, or within $\sim 3^{\prime}$ of each other on the sky. The Mergers are either listed in Joseph and Wright (1985) or possibly have polar rings, as in the case of NGC 660 and NGC 2146 (Schweizer et al. 1983).

Col. (6) Logarithm of the ratio of the $\mathrm{H} \alpha$ luminosity of the molecular gas mass, or the yield of young stars per unit mass of available gas, in units of $\mathrm{L}_{\mathrm{o}} / \mathrm{M}_{\mathrm{o}}$. Values have an overall uncertainty of $\pm 35 \%$. Values indicated by an asterisk are the galaxies for which we have determined extinction corrections from images of the [SIII] emission (Young, Kleinmann, and Allen 1988). The extinction corrected values of $\mathrm{L}(\mathrm{H} \alpha) / \mathrm{M}\left(\mathrm{H}_{2}\right)$, in the same units as those listed in column 6 above, are: NGC 520 (-1.63),NGC 660 (-0.76), NGC 1068 (-1.35), NGC $2146(-1.40)$, NGC 3034 (-1.21), and Arp 220 (-1.20).

Col. (7) Logarithm of the ratio of the far-infrared luminosity to the molecular gas mass, in units of $L_{0} / M_{0}$. Values have an overall uncertainty of $\pm 35 \%$.

Col. (8) Ratio of the IRAS 60 to $100 \mu \mathrm{m}$ flux densities from Table 3.

sen such that the nearest galaxy on the sky (within \pm 500 $\mathrm{km} \mathrm{s}^{-1}$ ) is at least $1.2 \mathrm{Mpc}$ distant. The angular separations to the nearest neighbors on the sky were determined using the Nearby Galaxies Atlas (Tully 1989), with velocities taken from the RC2.

Table 5 lists the mean high mass star formation efficiencies for the different environmental groups described above. While previous studies have reported that the ratio $L_{\mathrm{IR}} / \mathscr{O}\left(\mathrm{H}_{2}\right)$ is enhanced in merging systems (Young et al. 1986a, 1986b; Sanders et al. 1986; Solomon \& Sage 1988; Tinney et al. 1990), this is the first study which explores the star formation efficiency as a function of environment where the $\mathrm{H} \alpha$ luminosity is used to trace the high mass star formation. We find that both $L(\mathrm{H} \alpha) / \mathscr{M}\left(\mathrm{H}_{2}\right)$ and $L_{\mathrm{IR}} / \mathscr{M}\left(\mathrm{H}_{2}\right)$ are enhanced in the mergers. Interestingly, the mean SFE for the isolated galaxies is similar to that for the field galaxies, galaxy pairs, and Virgo early-type galaxies. Only the late-type spiral galaxies and the irregulars have mean ratios of both $L(\mathrm{H} \alpha) / \mathscr{M}\left(\mathrm{H}_{2}\right)$ and $L_{\mathrm{IR}} / \mathscr{L}\left(\mathrm{H}_{2}\right)$ that are comparable to the values for the mergers. The mean high mass star formation efficiency of the mergers is 4-8 times higher than that of the isolated galaxy sample, as measured by both $L(\mathrm{H} \alpha) / \mathscr{M}\left(\mathrm{H}_{2}\right)$ and $L_{\mathrm{IR}} / \mathscr{M}\left(\mathrm{H}_{2}\right)$. The $\mathrm{H} \alpha$ data therefore support the earlier results, based on the mean $L_{\mathrm{IR}} / \mathscr{M}\left(\mathrm{H}_{2}\right)$ in several environmentally-selected samples of galaxies, that the high mass star formation efficiency is enhanced by a significant amount in strongly interacting systems. Thus, environment appears to have a substantial effect on the global yield of high mass stars per unit mass of molecular gas in spiral galaxies. Since the mean high mass star formation efficiency for the pairs in our sample is not elevated, we agree with Solomon \& Sage (1988) that a strong interaction is required to boost the star formation efficiency. Clearly, it would be of interest to study the molecular and ionized gas content of a large sample of galaxy pairs with a range of separations to examine the star formation efficiencies as a function of the strength of the galactic encounter.

The observations presented here indicate that the efficiency of forming high mass stars is enhanced in merging systems relative to isolated galaxies, although the mechanism responsible for this enhancement is not clear. One such possibility, although by no means the only one, is cloud- 


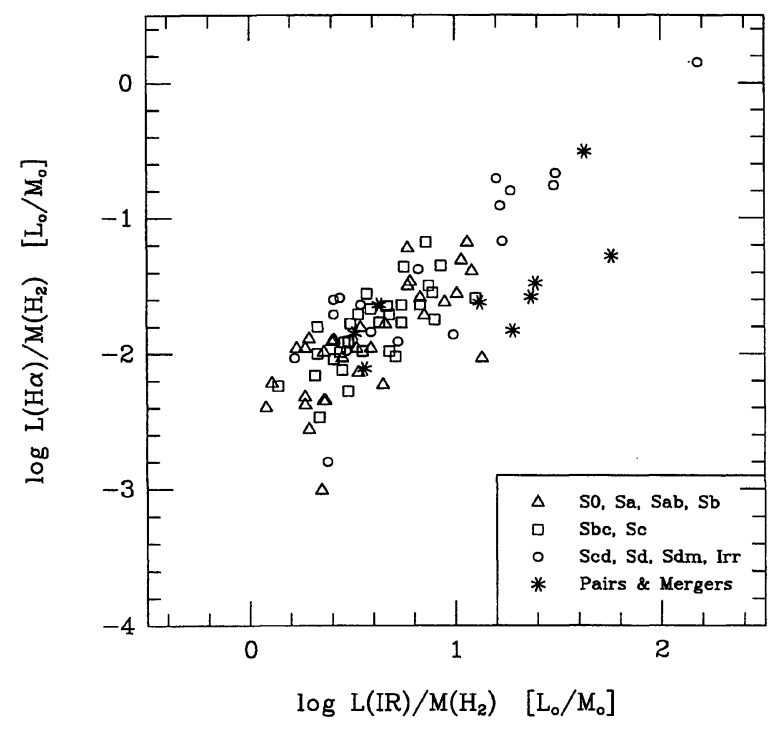

FIG. 11. Comparison of two measures of the star formation efficiency, that derived from the ratio of $\mathrm{H} \alpha$ luminosity to $\mathrm{H}_{2}$ mass with that derived from the ratio of the far-infrared luminosity to $\mathrm{H}_{2}$ mass. Galaxies are coded by morphological type as indicated. Only galaxies with inclinations less than $70^{\circ}$ are included.

cloud collisions, which may lead to the formation of high mass stars (Scoville et al. 1986), and whose rate has been shown to be enhanced for strong galaxy-galaxy interactions (Noguchi \& Ishibashi 1986; Olson \& Kwan 1990a, 1990b).

\subsection{Time scale for Cycling of Gas Through Stars}

There have been some suggestions in the literature of the depletion of gas in galaxies through star formation. This of course depends on the assumption that the low mass stars, which we do not observe, form in proportion to the high mass stars according to some IMF. It is the low mass stars which lock up mass into inert remnants, while the high mass stars are recycled into the ISM. If only high mass stars form, or if the IMF is weighted toward the high mass end, then gas depletion may not occur at all. For this reason, we refer here to the gas cycling time rather than the depletion time.

In Sec. 4.2 we described the relationship between the luminosity in high mass stars and the quantity of gas undergoing star formation [see Eq. (9)]. Additionally, Table 3 lists the global interstellar masses of molecular and atomic gas. Therefore, we can estimate the length of time that the present star formation rate can be sustained by the available supply of gas, under the assumption that the star formation rate persists into the future. This gas cycling time is given by the reciprocal of the star formation efficiency for an adopted IMF.

Figure 13 shows the global $\mathrm{H} \alpha$ luminosity compared with the total amount of neutral interstellar gas mass in molecular and atomic form. The dashed lines indicate the gas cycling times, under the assumption of a modified Miller-Scalo IMF between $0.1 \mathscr{M}_{0}$ and $100 \mathscr{M}_{0}$, as described in Sec. 4.2. For the galaxies in this sample, the majority of the cycling times fall between $10^{9}$ and $10^{10}$ years. It is important to realize that some of the neutral gas, especially the $\mathrm{H} \mathrm{I}$ in the outer parts of galaxies, may not be associated with star formation. Furthermore, correcting the $\mathrm{H} \alpha$ luminosities for internal extinction would increase the luminosities and decrease the cycling times. Therefore, the cycling times could in fact be shorter than those indicated in Fig. 13. We note that it is also possible that the cycling time will vary with radius in a galaxy, that regions with high star formation efficiency will cycle the gas in shorter times than regions of low efficiency.

To verify our methodology and results, it is important to compare the gas cycling times estimated from the $\mathrm{H} \alpha$ luminosity in Fig. 13 with gas cycling times estimated from the total luminosity, following Scoville \& Young (1983). Under the assumption that most of the luminosity of a galaxy is produced by $\mathrm{O}, \mathrm{B}$, and $\mathrm{A}$ stars, and that these stars process $13 \%$ of their mass through the CNO cycle while on the main sequence (Schwarzschild 1958), an estimate of the star formation rate is given by

$$
\mathrm{SFR}_{0, \mathrm{~B}, \mathrm{~A}}=7.7 \times 10^{-11} L_{\mathrm{tot}}\left[\mathscr{M}_{0} / \mathrm{yr}\right],
$$

where $L_{\text {tot }}$ is the total luminosity. Figure 14 shows a comparison of the total luminosity, determined from the farinfrared and blue bands (see Table 3), with the total mass of gas in molecular and atomic form. The dashed lines indicate the times in which the available gas will be cycled through stars given the present star formation rates. As in Fig. 13, the majority of the galaxies have cycling times between $10^{9}$ and $10^{10}$ years. Thus, for a $10^{10}$ year old disk, the present level of star formation can be sustained into the future for a time which is comparable to the current age of the disk.

\section{CONCLUSIONS}

We have obtained CCD images of 120 galaxies in the light of $\mathrm{H} \alpha$ and the $R$-band continuum in order to determine the distribution of star formation within spiral galaxies.

(1) We find a small but significant variation in the mean $\mathrm{H} \alpha$ surface brightness as a function of type for spiral galaxies along the Hubble sequence. The Sd-Ir galaxies have a mean $\mathrm{H} \alpha$ surface brightness which is 1.4 times higher than for Sbc-Scd galaxies, and 2-3 times higher than for $\mathrm{Sa}-\mathrm{Sb}$ galaxies.

(2) The star formation rate per unit mass of molecular gas, deduced from the global ratio $L(\mathrm{H} \alpha) / \mathscr{L}\left(\mathrm{H}_{2}\right)$, shows no significant variations in the mean as a function of morphological type for the spiral galaxies with types Sa-Sc in our sample. We find that this ratio varies by roughly an order of magnitude within each type, while the $\mathrm{H} \alpha$ luminosities for the galaxies themselves range over four orders of magnitude for the entire sample. It therefore appears that, at the current epoch, galactic disks for bright spiral galaxies are globally converting gas into stars with roughly the same mean efficiency.

(3) The high mass star formation rate per unit mass of molecular gas is enhanced in mergers relative to isolated spirals by a factor of $\sim 4$ (uncorrected for extinction). This confirms earlier results based on the ratio $L_{\mathrm{IR}} / \mathscr{O}\left(\mathrm{H}_{2}\right)$ that the yield of high mass stars per unit mass of molecular gas is elevated in merging and strongly interacting systems. The 


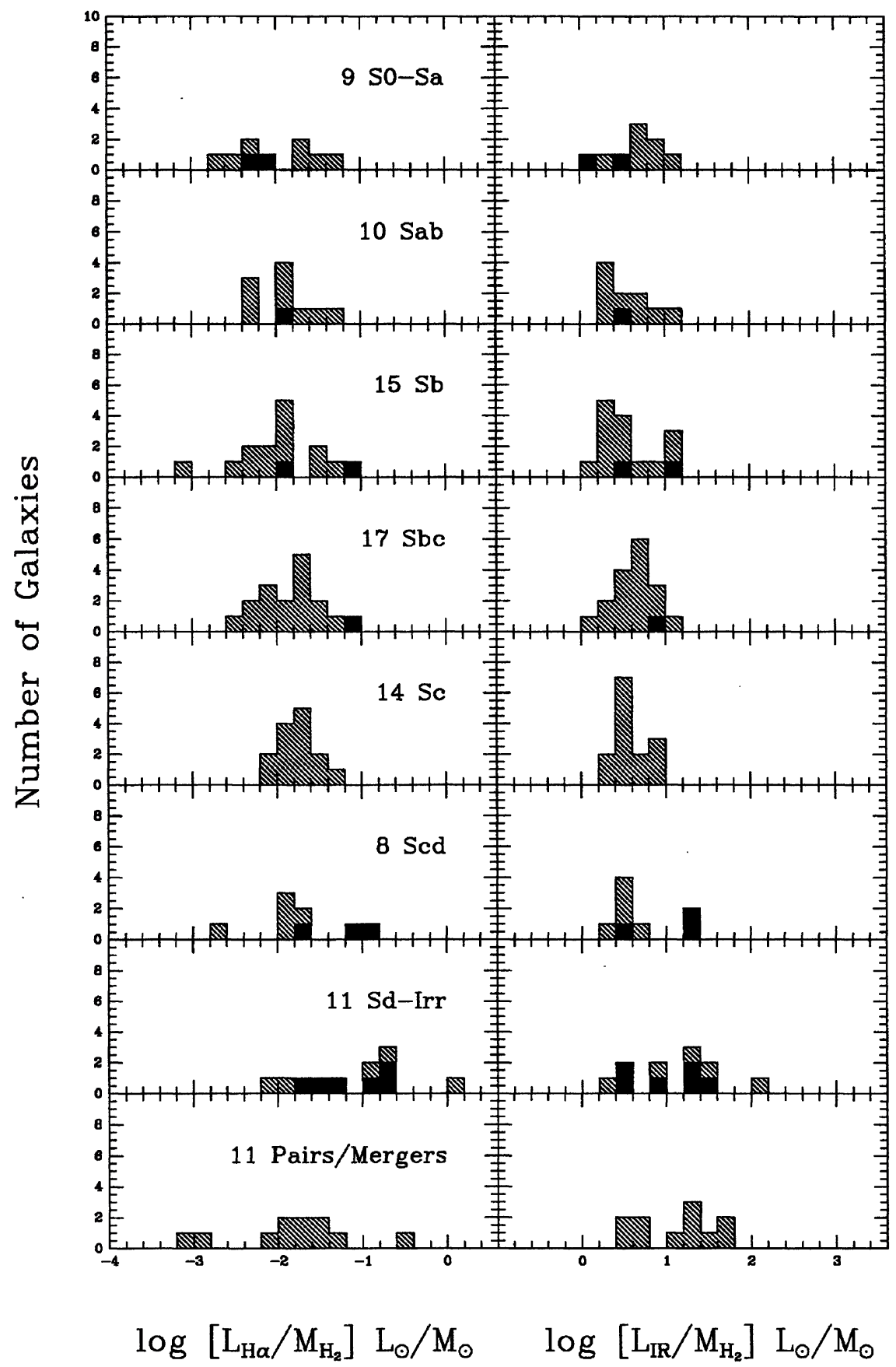

FIG. 12. Histograms of the star formation efficiency as a function of morphological type measured from the ratio of H $\alpha$ luminosity to molecular gas mass (left-hand panels) and from the ratio of far-infrared luminosity to molecular gas mass (right-hand panels). The solid portion of each histogram indicates lower limits to the star formation efficiency (based on upper limits to the $\mathrm{CO}$ integrated intensity). Only galaxies with inclinations less than $70^{\circ}$ are included.

$\mathrm{H} \alpha$ images also demonstrate that the elevated $\mathrm{H} \alpha$ luminosities arise in discrete $\mathrm{H}$ II regions as opposed to active nuclei.

(4) The comparison of the $\mathrm{H} \alpha$ and IR luminosities with the molecular gas masses for the spiral galaxies in our sample leads to further evidence that the IR luminosity of a galaxy is a measure of the rate of high mass star formation, and that old stars do not contribute significantly to the far-IR luminosity measured by IRAS. If old stars did contribute sig- nificantly to the IR luminosity of a galaxy, this would be most apparent in the early-type bulge-dominated systems relative to the systems with small bulges. However, the similarity of the ratios $L(\mathrm{H} \alpha) / \mathscr{M}\left(\mathrm{H}_{2}\right)$ and $L_{\mathrm{IR}} / \mathscr{C}\left(\mathrm{H}_{2}\right)$ for Sa versus $\mathrm{Sc}$ galaxies, and the knowledge that the $\mathrm{H} \alpha$ luminosity generally arises in discrete, identifiable $\mathrm{H}$ II regions, indicates that old stars do not contribute significantly to the IR luminosity of a spiral galaxy. 
TABLE 5. Summary of star formation efficiencies.

\begin{tabular}{|c|c|c|c|c|}
\hline $\begin{array}{l}\text { Category } \\
\text { (1) }\end{array}$ & $\begin{array}{l}\text { Subset } \\
\text { (2) }\end{array}$ & $\begin{array}{l}\text { Number of } \\
\text { Galaxies } \\
\text { (3) }\end{array}$ & $\begin{array}{c}<\log \left[\mathrm{L}(\mathrm{H} \alpha) / \mathrm{M}\left(\mathrm{H}_{2}\right)\right]> \pm \sigma \\
{\left[\mathrm{L}_{\mathrm{o}} \mathrm{M}_{\mathrm{o}}\right]} \\
(4)\end{array}$ & $\begin{array}{c}<\log \left[\mathrm{L}(\mathrm{IR}) / \mathrm{M}\left(\mathrm{H}_{2}\right)\right]> \pm \sigma \\
{\left[\mathrm{L}_{(5)} \mathrm{M}_{\mathrm{o}}\right]} \\
(5)\end{array}$ \\
\hline Morphological & SO-SOa & 3 & $-1.96 \pm 0.23$ & $0.65 \pm 0.07$ \\
\hline \multirow[t]{8}{*}{ Type } & $\mathrm{Sa}$ & 5 & $-1.89 \pm 0.20$ & $0.65 \pm 0.20$ \\
\hline & $\mathrm{Sab}$ & 9 & $-1.88 \pm 0.11$ & $0.53 \pm 0.09$ \\
\hline & $\mathrm{Sb}$ & 15 & $-1.96 \pm 0.12$ & $0.55 \pm 0.08$ \\
\hline & $\mathrm{Sbc}$ & 18 & $-1.82 \pm 0.08$ & $0.61 \pm 0.06$ \\
\hline & $\mathrm{Sc}$ & 14 & $-1.76 \pm 0.06$ & $0.58 \pm 0.05$ \\
\hline & Scd & 8 & $-1.73 \pm 0.20$ & $0.70 \pm 0.13$ \\
\hline & Sd-Sm & 7 & $-1.30 \pm 0.18$ & $0.79 \pm 0.16$ \\
\hline & Irr & 4 & $-0.78 \pm 0.41$ & $1.53 \pm 0.24$ \\
\hline \multirow[t]{8}{*}{ Environment } & Isolated & 10 & $-1.92 \pm 0.10$ & $0.53 \pm 0.07$ \\
\hline & Field & 35 & $-1.80 \pm 0.09$ & $0.66 \pm 0.06$ \\
\hline & Pairs & 6 & $-1.91 \pm 0.12$ & $0.70 \pm 0.16$ \\
\hline & Virgo $\leq \mathrm{Sb}$ & 14 & $-1.89 \pm 0.10$ & $0.54 \pm 0.08$ \\
\hline & Virgo Sbc-Sc & 11 & $-1.82 \pm 0.09$ & $0.58 \pm 0.09$ \\
\hline & Virgo Scd-Ir & 12 & $-1.30 \pm 0.14$ & $0.91 \pm 0.12$ \\
\hline & Mergers & 5 & $-1.34 \pm 0.23$ & $1.44 \pm 0.12$ \\
\hline & Mergers & $9 *$ & $-1.30 \pm 0.14$ & $1.22 \pm 0.13$ \\
\hline
\end{tabular}

Notes to Table 5

Col. (1) and (2) Characteristics of the galaxies examined.

Col. (3) Number of galaxies in each subsample with inclinations $<70^{\circ}$ (see Table 4). Pairs and Mergers were not included in the morphological type categories. Galaxies whose classification includes the designation peculiar were included. The Merger category indicated by the asterisk $(*)$ includes 4 galaxies with high extinction (NGC 520, NGC 660, NGC 2146, and Arp 220) for which extinction corrections to the H $\alpha$ luminosities were included (cf. Young, Kleinmann, and Allen 1988). The merger category without the asterisk does not include these 4 galaxies.

Col. (4) and (5) Mean values of the star formation efficiency, measured in both $\mathrm{H} \alpha$ and the IR. The means values listed refer to galaxies with inclinations less than $70^{\circ}$. The uncertainties listed indicate the accuracy of the mean value.

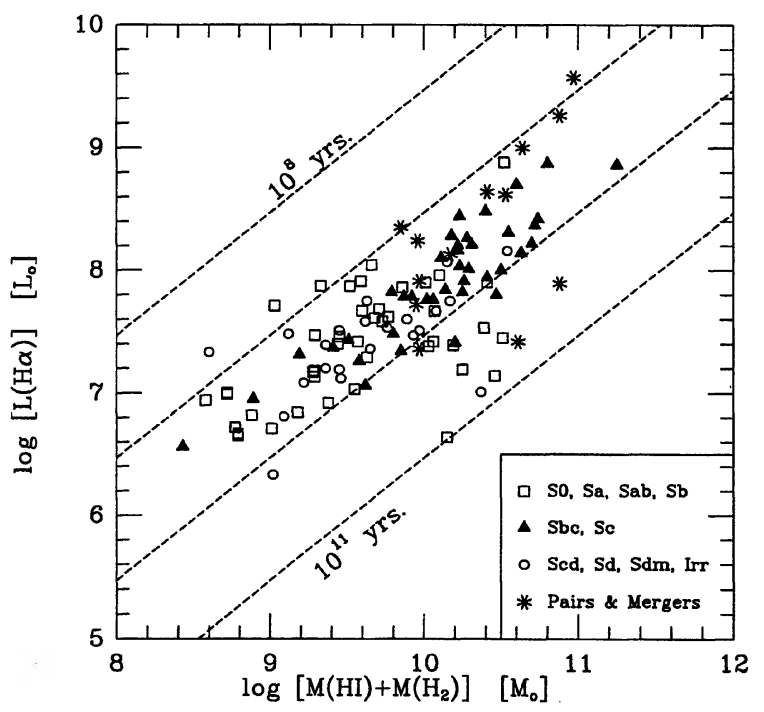

FIG. 13. Comparison of the global $\mathrm{H} \alpha$ luminosities with the total neutral interstellar gas masses, with galaxies of differing morphological type indicated. The dashed lines indicate the gas cycling times assuming that the total star formation rate is derived from the $\mathrm{H} \alpha$ luminosity [see Eq. (9b)]. Note that the majority of the cycling times fall between $10^{9}$ and $10^{10}$ years.

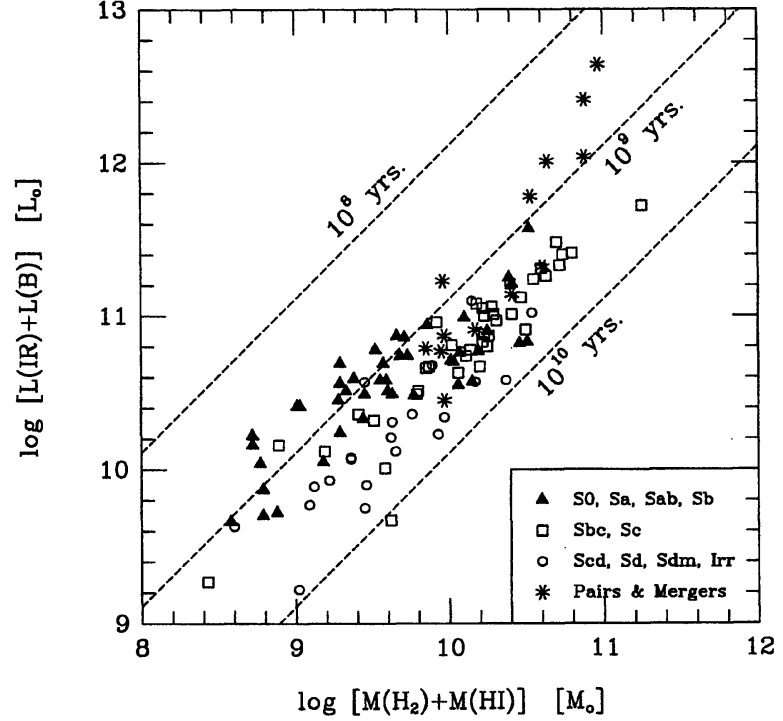

FIG. 14. Comparison of the total luminosity (far-infrared+blue) with the total neutral interstellar gas masses, with galaxies of differing morphological type indicated. The dashed lines indicate the gas cycling times assuming that the star formation rate is derived from the total luminosity [see Eq. (10)]. Note that the majority of the cycling times fall between $10^{9}$ and $10^{10}$ years. 
We wish to thank the Director of NOAO for a generous allottment of time on the now-retired No. 1-0.9 m telejscope, and the staff of NOAO for outstanding support at the telescope and in downtown Tucson. We extend special thanks to Bill Schoening, George Jacoby, Jeanette Barnes, Brett Goodrich, and Rob Seaman, as well as those in the photo lab. We also wish to thank Shuding Xie for assisting at the telescope during the first observing run, Shannon Hall for determining the isolated galaxy sample used here, and Erica McConnell for loading more than 200 CCD images from a maze of 33 nine-track tapes onto the computer. This study was begun with support from a Sloan Foundation Fellowship. J.Y. also wishes to thank the JCMT and the University of Hawaii for excellent support as a Visiting JCMT Fellow during 1991 when the data analysis was in progress. Finally, we wish to thank the anonymous referee who made a number of comments which helped improve the manuscript.

\section{REFERENCES}

Arp, H. 1966, ApJS, 14, 1

Barnes, J., \& Hayes, D. 1984, The IRS Standard Star Manual (NOAO, Tucson)

Bagnuolo, W. G. 1976, Ph.D. thesis, California Inst. of Technology

Benvenuti, P., Capaccioli, M., \& D’Odorico, S. 1975, A\&A, 41, 91

Bloemen, J. B. G. L., et al. 1986, A\&A, 154, 25

Boroson, T. 1981, ApJS, 46, 177

Bothun G., Lonsdale, C. J., \& Rice, W. 1989, ApJ, 341, 129

Bushouse, H. A. 1987, Ph.D. thesis, University of Illinois

Cohen, J. G. 1976, ApJ, 203, 587

Deharveng, G., J.-M., Sasseen, T. P., Buat, V., Bowyer, S., Lampton, M., \& Wu, X. 1994, A\&A, 289, 715

De Jong, T., et al. 1984, ApJ, 278, L67

De Jong, T., Klein, U., Wielebinski, R., \& Wunderlich, E. 1985, A\&A, 147, L6

de Vaucouleurs, G., de Vaucouleurs, A., \& Corwin, H. G. 1976, Second Reference Catalogue of Bright Galaxies (University of Texas Press, Austin)

Dennefeld, M., \& Stasinska, G. 1985, A\&A, 118, 234

Devereux, N., \& Young, J. S. 1990a, ApJL, 350, L25

Devereux, N, \& Young, J. S. 1990b, ApJ, 359, 42

Devereux, N., \& Young, J. S. 1991, ApJ, 371, 515

Garcia-Vargas, M. L., Bressan, A., \& Diaz, A. I. 1995, A\&AS, 112, 13

Gavazzi, G., Cocito, A., \& Vettolani, G. 1986, ApJ, 305, L15

Helou, G., Soifer, B. T., \& Rowan-Robinson, M. 1985, ApJ, 298, L7

Hildebrand, R. H. 1983, QJRAS, 24, 267

Huchra, J. 1977, ApJ, 217, 928

Huchtmeier, W., Bregman, J., Hogg, D., \& Roberts, M. S. 1988, A\&A, 198, L17

Huchtmeier, W., Richter, O.-G., Bohnenstengel, H.-D., \& Hauschildt, M. 1983, A General Catalogue of H I Observations of External Galaxies, ESO Preprint No. 250

Joseph, R. D., \& Wright, G. S. 1985, MNRAS, 219, 87

Kenney, J., \& Young, J. S. 1988, ApJS, 66, 261

Kenney, J. D. P., \& Young, J. S. 1989, ApJ, 344, 171

Kennicutt, R. C. 1983, ApJ, 272, 54

Kennicutt, R. C., \& Kent, S. M. 1983, AJ, 88, 1094

Kennicutt, R. C., Keel, W. C., van der Hulst, J. M., Hummel, E., \& Roettiger, K. A. 1987, AJ, 93, 1011

Kent, S. M. 1985, ApJ, 59, 115

Larson, R. B., \& Tinsley, B. M. 1978, ApJ, 219, 46

Lester, D. F., Dinerstein, H. L., \& Rank, D. M. 1979, ApJ, 232, 139

Lonsdale, C. J., \& Helou, G. 1987, ApJ, 314, 513

Lonsdale, C., Helou, G., Good, J., \& Rice, W. 1985, in Catalogued Galaxies and Quasars in the IRAS Survey (US GPO, Washington, D.C.)

Maloney, P., \& Black, J. 1988, ApJ, 325, 389

Noguchi, M., \& Ishibashi, S. 1986, MNRAS, 219, 305

Olson, K. M., \& Kwan, J. 1990a, ApJ, 349, 480

Olson, K. M., \& Kwan, J. 1990b, ApJ, 361, 426

Pagel, B. E. J., \& Edmunds, M. G. 1981, ARA\&A, 19, 77
Panagia, N. 1973, AJ, 78, 929

Pogge, R. W., \& Eskridge, P. B. 1987, AJ, 92, 291

Rand, R., Kulkarni, S. R., \& Rice, W. 1992, ApJ, 390, 66

Rengarajan, T. N., \& Verma, R. P. 1986, A\&A, 165, 300

Rice, W., Lonsdale, C. J., Soifer, B. T., Neugebauer, G., Kopan, E. L., Lloyd, L. A. De Jong, T., \& Habing, H. J. 1988, ApJ, 68, 91

Roberts, M. S. 1969, AJ, 74, 859

Rubin, R. 1985, ApJS, 57, 349

Rubin, R. 1988, ApJS, 68, 519

Rubin, V. C., \& Ford, W. K. 1986, ApJ, 305, L35

Sandage, A. 1986, A\&A, 161, 89

Sanders, D. B., \& Mirabel, I. F. 1985, ApJ, 298, L31

Sanders, D. B., Scoville, N., Young, J. S., Soifer, B. T., \& Danielson, G. 1986, ApJ, 305, LA5

Sanders, D. B., Young, J. S., Scoville, N., Soifer, B. T., \& Danielson, G. 1987, ApJ, 312, 15

Schoening, B. 1987, The CCD Direct Imaging Camera (NOAO, Tucson)

Schwarzschild, M. 1958, Structure and Evolution of the Stars (Dover, New

York), p. 276

Schweizer, F., Whitmoer, B. C., \& Rubin, V. C. 1983, AJ, 88, 909

Scoville, N., Sanders, D. B., \& Clemens, D. 1986, ApJL, 310, L77

Scoville, N. Z., \& Young, J. S. 1983, ApJ, 265, 148

Scoville, N., Yun, M., Clemens, D., Sanders, D., \& Waller, W. 1987, ApJS, 63,821

Smith, B. J., Harvey, P. M., Colome, C., Zhang, C. Y., DiFrancesco, J., \&

Pogge, R. W. 1994, ApJ, 425, 91

Solomon, P. M., \& Sage, L. 1988, ApJ, 334, 613

Solomon, P., Rivolo, R., Barrett, J., \& Yahil, A. 1987, ApJ, 319, 730

Telesco, C. M., \& Harper, D. A. 1980, ApJ, 235, 392

Thronson, H., et al. 1989, ApJ, 344, 747

Tinney, C. G., Scoville, N., Sanders, D., \& Soifer, B. T. 1990, ApJ, 362, 473

Toomre, A., \& Toomre, J. 1972, ApJ, 178, 623

Tully, R. B. 1989, Nearby Galaxies Catalogue (Cambridge University Press, Cambridge)

Vogel, S., Boulanger, F., \& Ball, R. 1987, ApJ, 316, L243

Waller, W. H., Gurwell, M., \& Tamura, M. 1992, AJ, 104, 63

Waller, W. H., Kleinmann, S. G., \& Ricker, G. 1988, AJ, 95, 1057

Wiklind, T., \& Henkel, C. 1989, A\&A, 225, 1

Wilson, C., \& Scoville, N. 1990, ApJ, 363, 435

Wynn-Williams, C. G., \& Becklin, E. 1974, PASP, 86, 5

Young, J. S., et al. 1986b, ApJ, 311, L17

Young, J. S., Kenney, J. D. P., Lord, S., \& Schloerb, F. P. 1984, ApJ, 287, L65

Young, J. S., Kleinmann, S. G., \& Allen, L. 1988, ApJ, 334, L63

Young, J. S., \& Knezek, P. 1989, ApJ, 347, L55

Young, J. S., Schloerb, F. P., Kenney, J. D. P., \& Lord, S. 1986a, ApJ, 304, 443

Young, J. S., \& Scoville, N. 1991, ARA\&A, 29, 581

Young, J. S., Xie, S., Kenney, J., \& Rice, W. L. 1989, ApJS, 70, 699

Young, J. S., et al. 1995, ApJS, 98, 219 


\section{$\mathrm{Sa} / \mathrm{Sab}$}

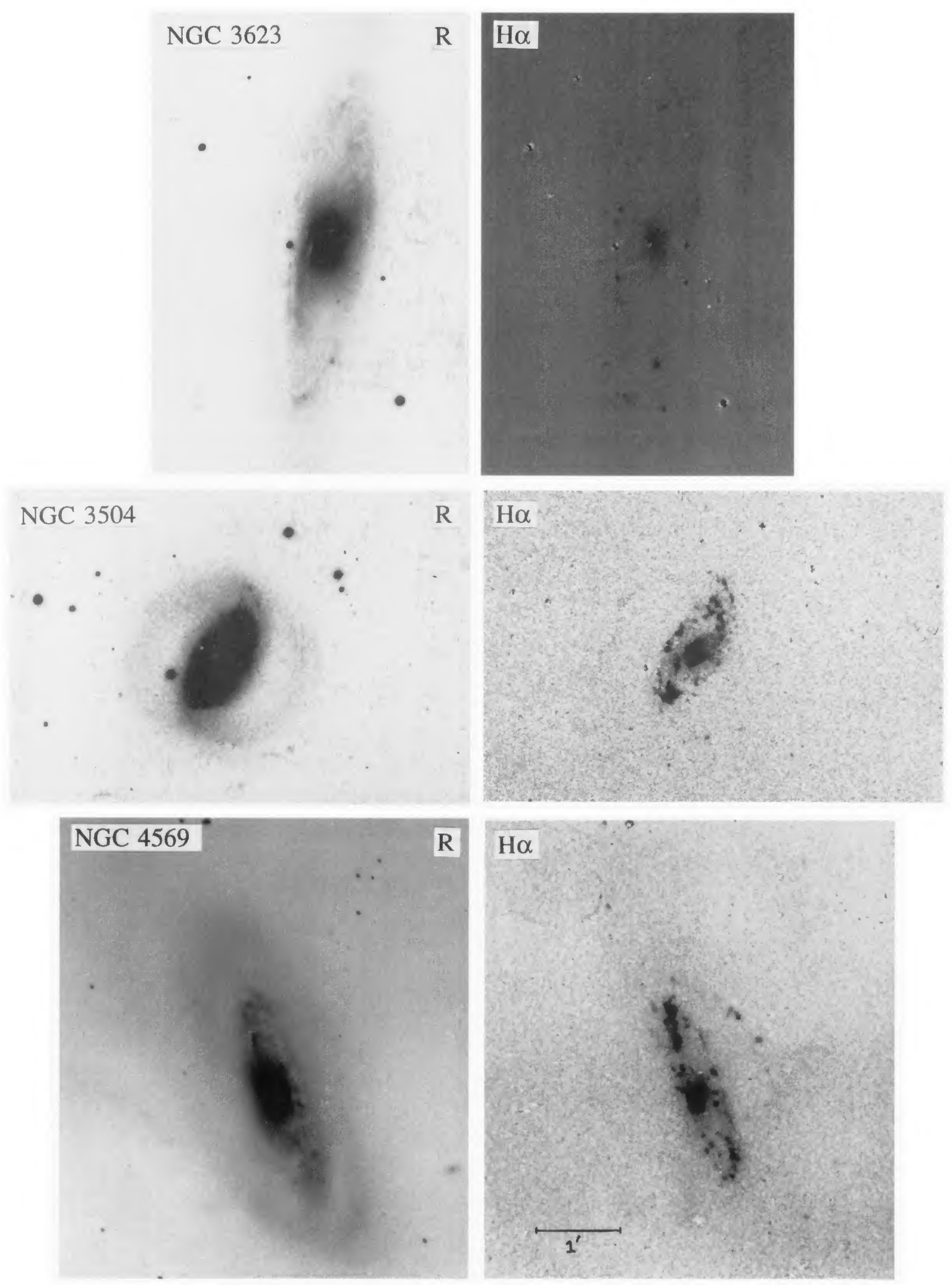

FIG. 2. $R$-band and continuum-free $\mathrm{H} \alpha+[\mathrm{N}$ II $]$ images of a subset of the galaxies in the present study, covering a wide range of morphological type. Young et al. (see page 1904) 
PLATE 86

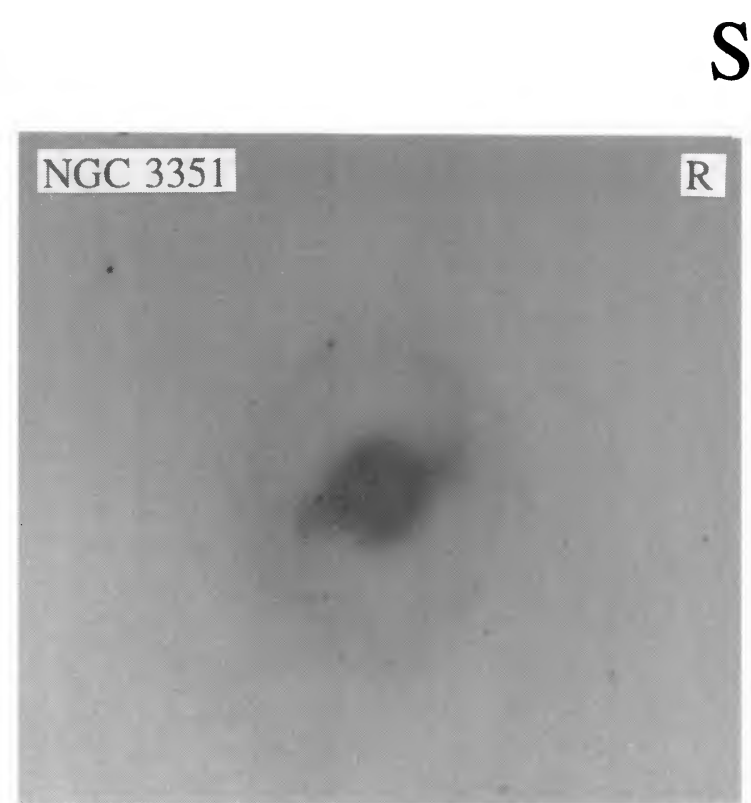

$\mathrm{Sb}$
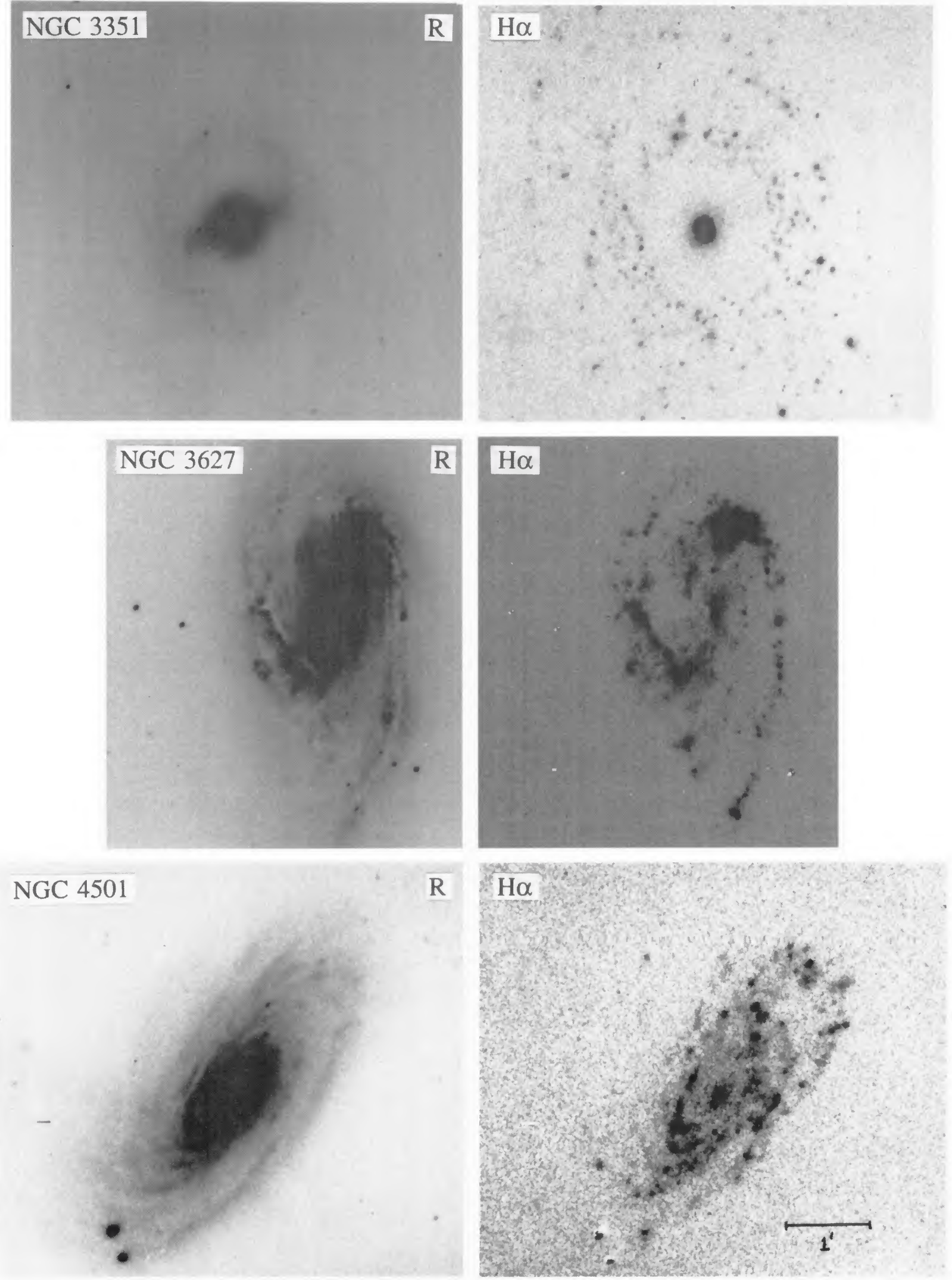

FIG. 2. (continued)

Young et al. (see page 1904) 


\section{Sbc}
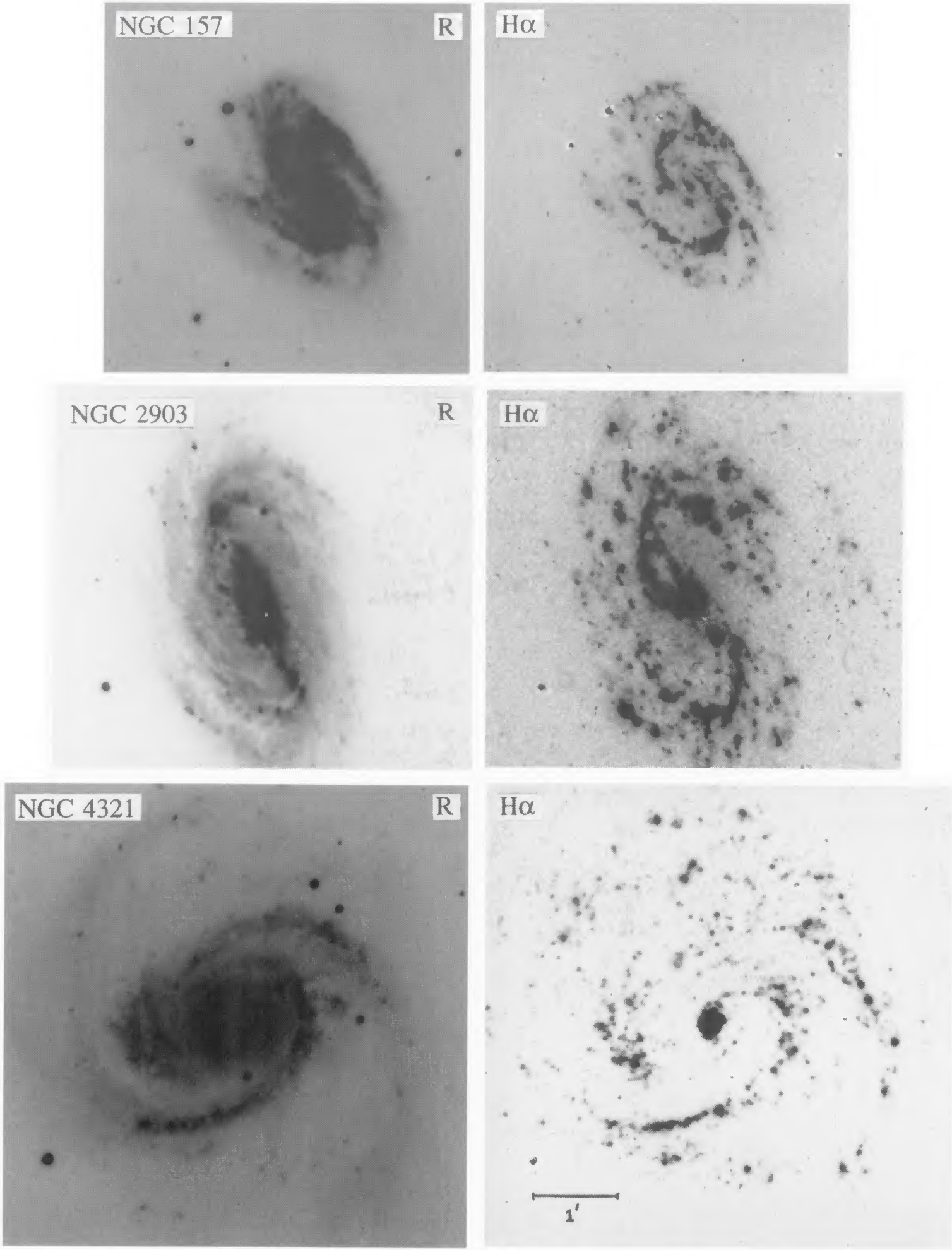

FIG. 2. (continued)

Young et al. (see page 1904) 


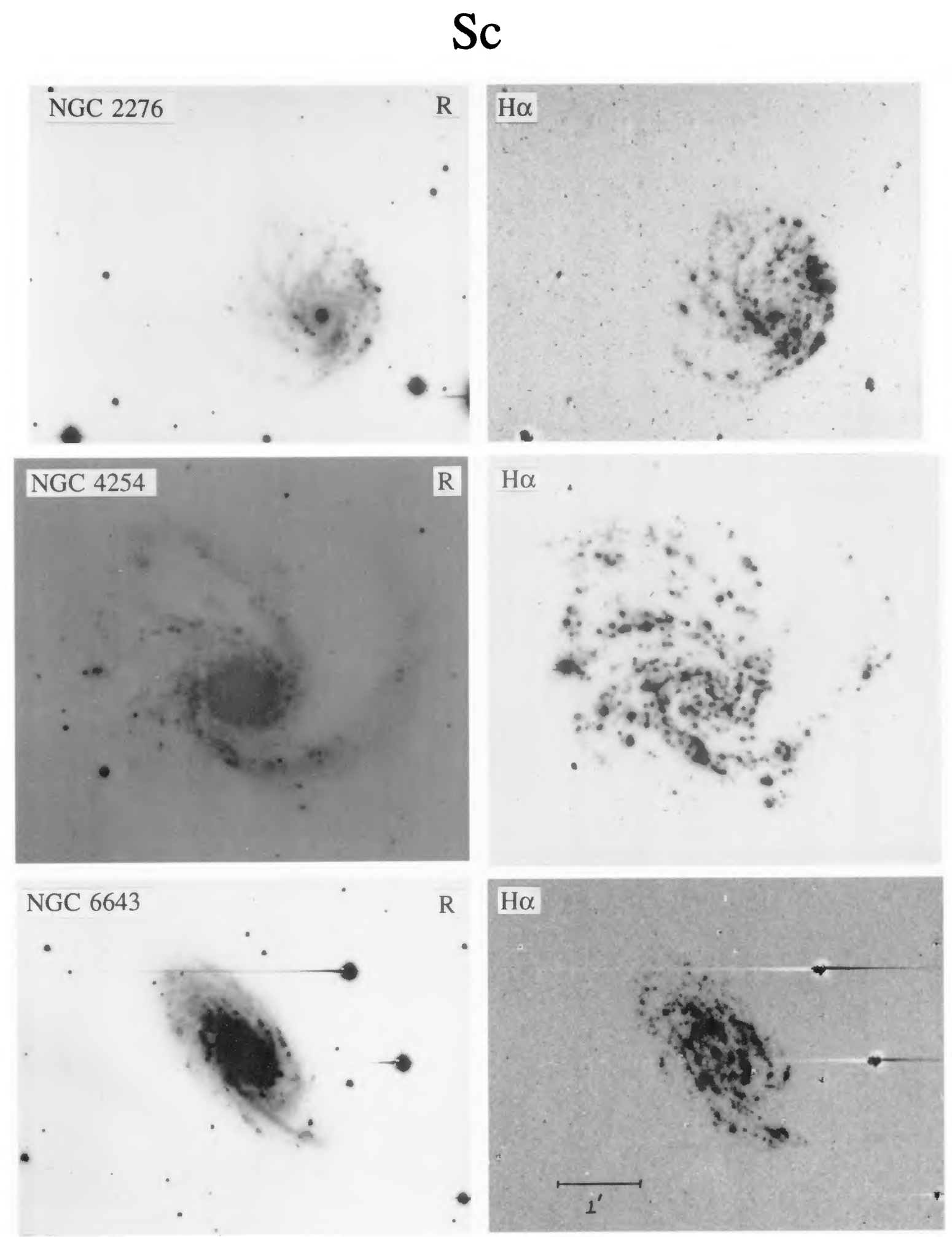

FIG. 2. (continued)

Young et al. (see page 1904) 


\section{Scd/Sd}
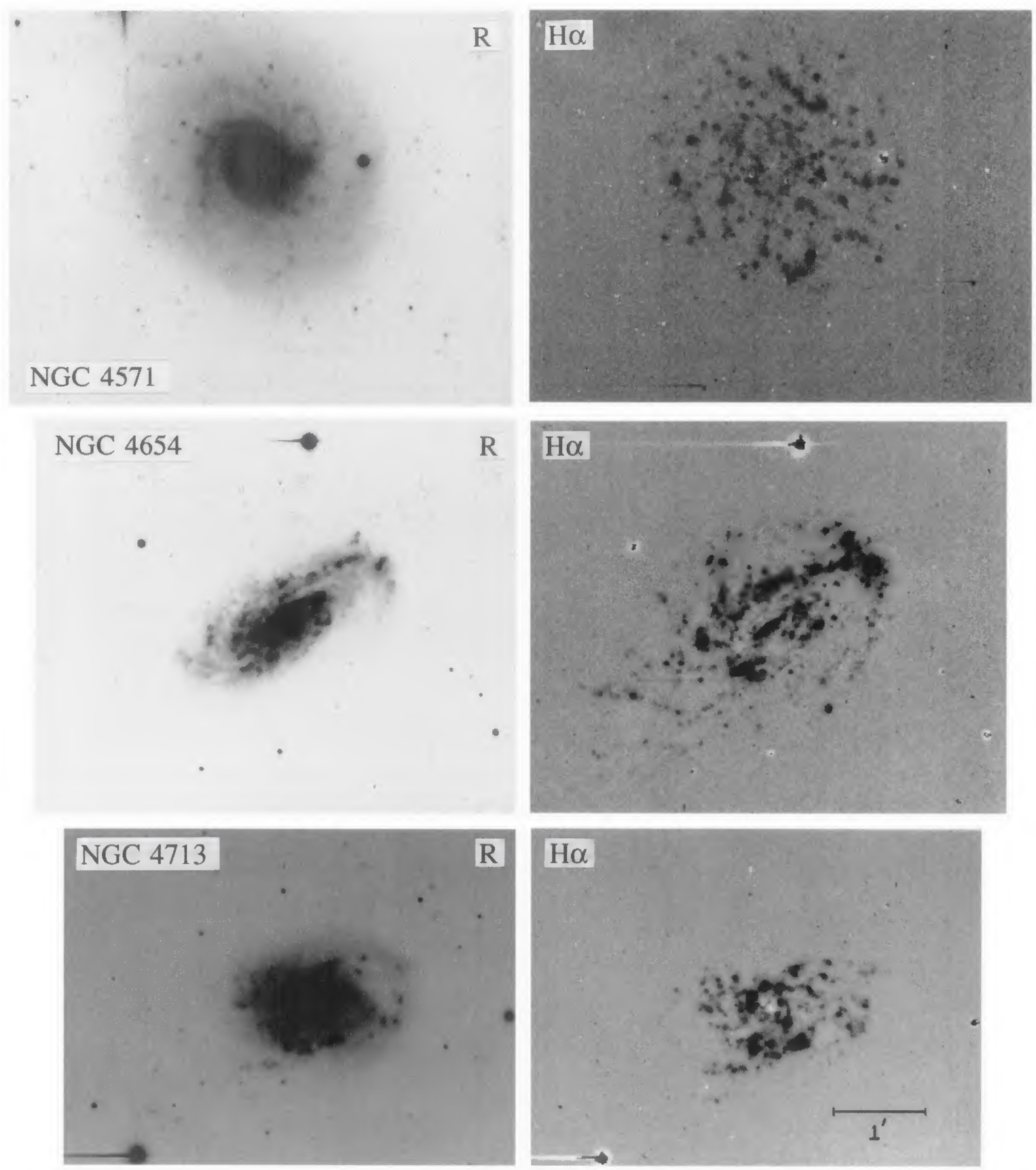

FIG. 2. (continued)

Young et al. (see page 1904) 


\section{$\mathrm{I} 0 / \mathrm{Im}$}
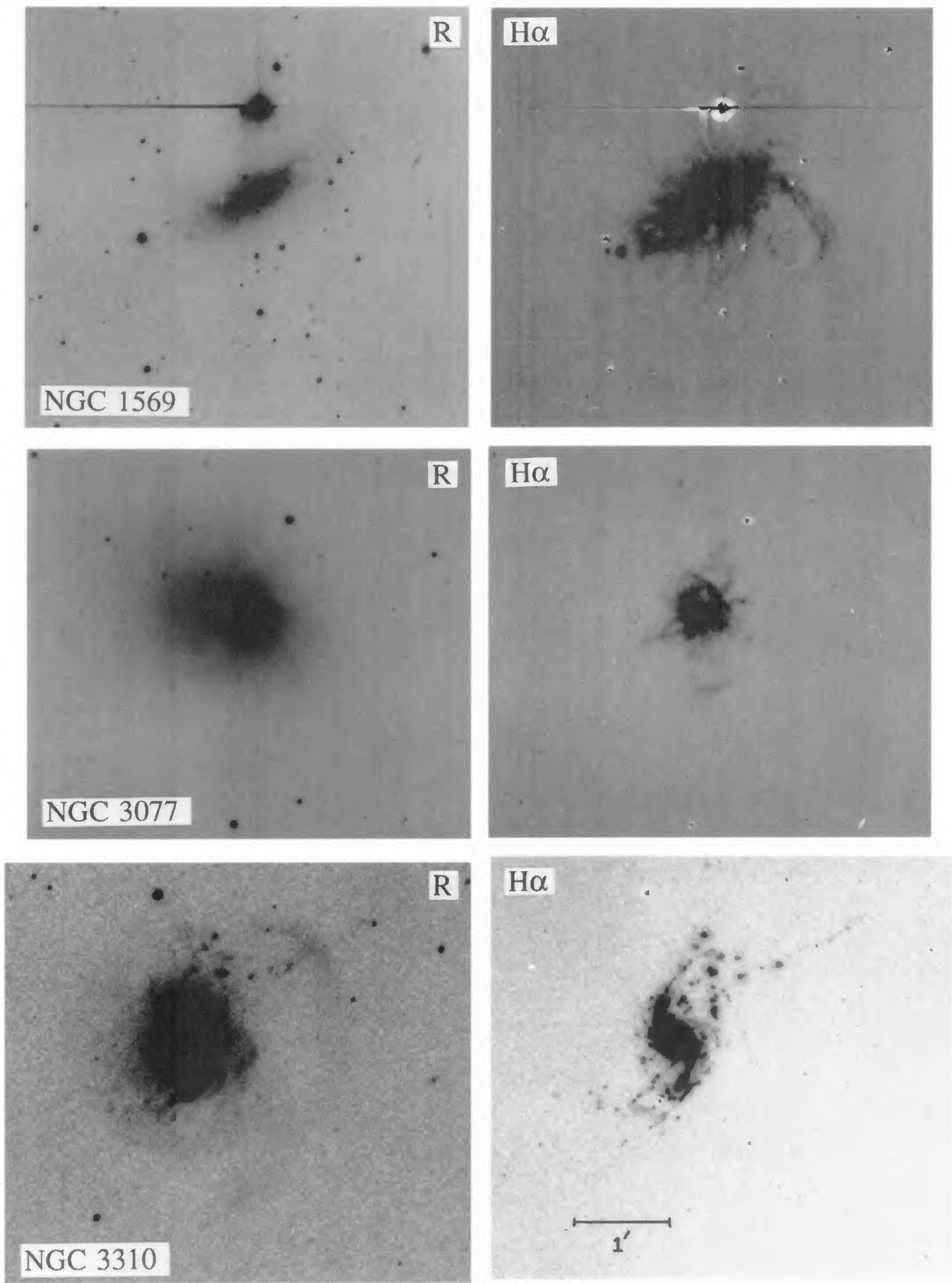

FIG. 2. (continued)

Young et al. (see page 1904) 